PSEUDOPOTENTIAL CALCULATIONS OF THE BAND STRUCTURE AND FERMI SURFACE OF MERCURY 


\title{
PSEUDOPOTENTIAL CALCULATIONS OF THE BAND STRUCTURE AND FERMI SURFACE OF MERCURY
}

\author{
by \\ JOHN CONRAD JONES, B.A., M.SC.
}

\author{
A Thesis \\ Submitted to the Faculty of Graduate Studies \\ in Partial Fulfilment of the Requirements \\ for the Degree \\ Doctor of Philosophy
}

McMaster University December 1970 
DOCTOR OF PHILOSOPHY (1971)

(Physics)

TITLE:

AUTHOR :

SUPERVISOR :

NUMBER OF PAGES: ix, 165

SCOPE AND CONTENTS:

The energy bands and Fermi surface of mercury have been calculated using local and non-local pseudopotentials. The non-local pesudopotentials were an approximation in which the repulsive potentials of the outer atomic core states were explicitly represented by non-local projection operators.

A search was made for the regions of parameter space where the pseudopotential generated a Fermi surface having a good fit to the experimental magneto-acoustic calipers and de Haas-van Alphen extremal cross sectional areas.

De Haas-van Alphen frequencies and cyclotron masses were calculated for symmetry planes using a local pseudopotential.

General questions of pseudopotential theory, the symmetry of the energy bands, the occurrence of degeneracies, and the influence of spin-orbit coupling are also considered. 


\section{ACKNOWLEDGEMENTS}

I am most grateful to my supervisor, Professor W. R. Datars for his guidance and help throughout the course of this research. Thanks are especially due to Professor L. M. Falicov for explaining pseudopotential theory to me, and for his guidance and advice at various times during this research.

I wish to thank McMaster University and the National Research Council of Canada for personal financial support. 
TABLE OF CONTENTS

CHAPTER

Page

I

INTRODUCTION

II

PSEUDOPOTENTIAI THEORY

1. Solution of the Pseudopotential

Eigenvalue Problem

2. Calculation of the Matrix Elements

3. Conversion to Atomic Units

4. The Evaluation of Integrals Containing Spherical Bessel Functions

5. Other Types of Pseudopotential

6. The Choice of a Suitable Approximation 21

7. Spin-orbit Coupling

8. Explicit Calculation of the Spin-orbit 31 Coupling Matrix elements

9. The Repulsive Potential of the Inner- 33 core states

III

GENERAL CHARACTERISTICS OF' THE ENERGY BANDS 46 OF MERCURY

1. Introouction

2. The Crystal structure and Brillouin zone of Mercury

3. The Free Electron Model

4. The Energy Gap at a General point on a 61 zone Face.

5. Group Theoretical Considerations 63

6. The Effect of Spin-orbit Coupling 70 
1. The Fermi Surface of Mercury 73

2. The General Approach of the Pseudo- 75 potential Calculations

3. The Coordinates in Reciprocal space of the Experimental Points

4. The Fermi Energy

5. The Choice of a Pseudopotential 86

6. Methods of Calculating Pseudopotential 88 Band Structures and Fermi surfaces for Mercury

7. Programs to Calculate Cross Sectional Areas of the Fermi Surface and Cyclotron Masses.

3. The Best Two Parameter Model (8 Point 105 Fit)

4. Three Regions of Parameter space

5. Tests to Determine the symmetry Character of the Energy Bands at Special Points in the Brillouin zone

6. The Order of Magnitude of the Spinorbit Interaction on the conduction bands of mercury.

7. Discussion

8. Comparison with Previous Calculations of the Fermi Surface of Mercury 


\section{LIST OF FIGURES}

FIGURE

Page

(II. I)

Form Factors of Atomic Wave Functions

35

$(I I .2)$

Form Factor of Unscreened Atomic Poten-

42 tial, and Hartxee dielectric function

(II.3)

Form Factors of screened Atomic potentials 45

(III.1)

The Brillouin Zone of Mercury, and the

52

'basic section' of the zone.

(III. 2)

Free Electron Energy Bands

(III.3)

Free Electron Energy Bands

56

(III.4)

Energy Bands for the Brand-Rayne Local

58

Pseudopotential

(III. 5)

Energy Bands for the Brandt-Rayne Local

59

Pseudopotential

(IV.1)

The System of Euler Angles used in the

Programs that Calculated Cross Sectional

Areas of the Fermi Surface.

(V.I)

(V.2)

(V.3)

Energy Bands for the 'best 8 point local 106 pesudopotential'

Energy Bands for the 'best 8 point local 107 pseudopotential.'

B arm dHvA frequencies, Trigonal-Bisec- 109 trix plane

(V.4) B arm cyclotron masses, Trigonal-Bisec- 110 trix plane

(V.5) Second Zone Lens dHvA Erequencies, Trigonal-Bisectrix plane

Trigonal-Bisectrix plane

(V.7) Second Zone Jens dHvA frequencies, Trigonal-Binary plane 
Second Zone Lens Cyclotron masses,

(V.9) Second Zone tuens dHvA frequencies, Tri-

Second zone Lens cyclotron masses, Trigonal plane

(V.11) Energy Bands for a class A non-local pseudopotential

(V.12) Energy Bands for a Class A non-local pseudopotential

$(\mathrm{V} .13)$

Energy Bands for a Class B non-local pseudopotential pseudopotential

The minimum $\beta$ arm cross section for the local pseudopotential $\mathrm{C}(\mathrm{O}, \mathrm{O})$

(V.16) The 'large section' of the lens for the local pesudopotential $\mathrm{C}(0,0)$

(V.17) The 'up section' of the lens for the local 138 pseudopotential c $(0,0)$

(V.18) The 'side section' of the lens for the local pseudopotential $\mathrm{C}(\mathrm{O}, \mathrm{O})$

(V.19) The 'minimum $\tau$ section' for the local pseudopotential C $(0,0)$

(V.20) The 'T face opening' for the local pseudopotential $\mathrm{C}(\mathrm{O}, \mathrm{O})$

(V.21) The 'X face opening' for the local pseudo- 142 potential $\mathrm{C}(0,0)$ 


\section{LIST OF TABLES}

TABIE

PAGE

(II.1) Comparison of Fouriex Transforms of the

Herman-Skillman Atomic Wave Functions

for Mercury

(II.2) The Effective Repulsive Potential of Inner- 39 core s-states

(III.1) Crystallographic Data for Hg 54

(III.2) The character Table for the Group $\overline{3} m \quad 64$

(IV.1) Experimental Calipers of the Fermi surface 79

(IV.2) The Coordinates of Important Symmetry Points 81 on the Brillouin Zone

(IV.3) The coordinates of the points on the Fermi 82 Surface that were used in the Fitting procedure

(IV.4) The Scalar products between two sets of unit 96 vectors in terms of Euler angles

(V.1) Examples of 8 Point Fits 102

(V.2) Examples of 14 Point Fits 104

(V.3) Class A Pseudopotentials (8 Point Fits) 1.8

(V.4) Class A Pseudopotentials (14 Point Fits) 120

(V.5) A Set of Class B Pseudopotentials 129

(V.6) Class C Pseudopotentials $\quad 131$

(V.7) Computed dHvA Frequencies and Cyclotron 134

Masses for Various Non-local Pseudopotentials

(V.8) Computed dHvA Frequencies and Cyclotron

143

Masses for a Class B Pseudopotential

(V.9) Symmetry Analysis for a Class A Pseudom 145 potential 
(V.10) Symmetry Analysis for a Class C Pseudopotential

(V.12) The Effect of Spin-orbit Coupling on

150

(V.13) Comparison of tocal Psevidopotential

157

(V.14) Comparisons of Extremal Areas for

158 Model Fermi. Surfaces with Experimental dHvA areas 


\section{CHAPTER I}

\section{INTRODUCTION}

In recent years there have been a considerable number of experimental investigations into the Fermi surface of mercury and the topology and shape of the surface are now fairly well known, although a few dimensions still remain uncertain. There have also been several calculations of the Fermi surface using different theoretical approaches. The pseudopotential method for mercury was first used by Brandt and Rayne (1966), while Dishman and Rayne (1968) carried out a pseudopotential calculation which included spin-orbit coupling. Bogle, Coon, and Greniex (1969) have also reported a pseudopotential calculation for mercury.

The only first principles calculation that has been reported for the energy bands and Fermi surface of mercury is the RAPW calculation of Keeton and Loucks (1966).

Pseudopotentials are usually determined by fitting a few disposable parameters to selected experimental data. and this was the method used in all the cases that have been mentioned. But in 1965 Heine and Animalu published tables of theoretical pseudopotential form factors for 25 elements including mercury. These tables indicated a particular region 
of parameter space for the local pseudopotential coefficients in mercury.

The pseudopotentials of Brandt and Rayne, Dishman and Rayne, and of Bogle, Coon, and Gxenier, all differed somewhat from one another, but they were all simple local pseudopotentials (except for Dishman and Rayne who included spinorbit coupling with a local pseudopotential) and they were all in the same general area of parameter space as had been given by Heine and Animalu.

All the theoretical calculations gave the same topology and shape for the Fermi surface but there were large variations in the calculated dimensions of the surface, and in no case were the theoretical dimensions in particularly good agreement with experiment.

Although there are quite strong resemblances between the pseudopotential energy bands and the relativistic bands there are also important differences, in particular the relativistic energy bands have a significantly higher Fermi energy.

All the previous pseudopotential calculations used a local pseudopotential, but this is an approximation and in general the pseudopotential is a non-local operator. In their work on zinc and cadmium Stark and Falicov (1967) tried local and non-local approximations and found that the nonlocal pseudopotentials gave much better fits to the experimental Fermi surface. The work described in this thesis was 
an attempt to calculate a more accurate Fermi surface of mercury by using non-local pseudopotentials similar to those used by Stark and Falicov.

In this investigation it was assumed that the area of parameter space indicated by Heine and Animalu might possibly be wrong, and a large region of parameter space was explored in an empirical fashion. All the regions which were found to be interesting were examined in some detail. Particular attention was paid to the structure of the energy bands because of the differences that existed between the local pseudopotential and the RAPW energy bands. 


\section{CHAPTER II}

\section{PSEUDOPOTENTIAL THEORY}

It has been found that in most metals, semimetals, and semiconductors that the energy bands for the highest energy states, which correspond to the outermost atomic electrons, are a recognizable distortion of the nearly free electron structure. The energy bands are most free-electron like in the simple metals, in the case of semimetals and semiconductors the distortions from the free electron band structure are much greater.

The evidence for these facts is that in most metals which have been studied the Fermi surface can be recognized as a distortion of the free electron sphere. This suggests that the conduction electrons of the metal are behaving very much like free electrons being weakly scattered by the ionic cores. In phenomenological terms it appears as if the conduction electrons are satisfying a schrödinger equation in which the dominant term is the kinetic energy operator $T$, with a comparatively weak pseudopotential w replacing the usual. potential energy operator $\mathrm{V}(\underline{\underline{r}})$

Pseudopotential theory provides a rigorous justification for this substitution, and a good approximation for the conduction energy bands of a metal can often be obtained 
by solving a schrödinger equation of the form,

$$
[T+W]\left|\phi_{k}>=E_{k}\right| \phi_{k}>
$$

in which $W$ is the weak pseudopotential, and $\phi_{\mathrm{k}}$ is a pseudowavefunction which is related to the true wavefunction $\psi_{\mathrm{K}}$. The band structures of the group IV semi-conductors and the III-V compounds have been probed by optical interband transitions, and these structures could also be interpre-ted in pseudopotential terms (Brust 1964, Cohen and Bergstresser 1966). Fermi surface studies coupled with band structure calculations on the semimetals $\mathrm{As}, \mathrm{Sb}, \mathrm{Bi}$, (Cohen, Falicov, and Golin (1964), Briestley, WindmilJer, Ketterson, and Ekstein (1967), Lin and Falicov (1966)), have indicated that in these substances also a pseudopotential approach is very useful.

In metals of the transition, raxe earth, and actinide groups the situation is more complicated because of the existence of incomplete inner $\mathrm{d}$ and $f$ shells.

If pseudopotentials can be successfully applied it implies that the ions of the solid are behaving very much like weak scattering centres so far as the electrons belonging to the highest energy states are concerned. This is at first somewhat surprising because the deep potential wells of the ionic cores are not weak scattering centres.

If a phase shift analysis is carried out, the scattering can be expressed in terms of the phase shift $\eta_{\ell}$. 
where,

$$
n_{\ell}=p_{\ell} \pi+\delta_{\ell}
$$

In this equation the integer $p_{\ell}$ which has been chosen so that $\left|\delta_{\ell}\right|<\frac{1}{2} \pi$, counts the number of internal radial nodes. Since the usual phase shift formula for the scattering only involves $\exp \left(2 i n_{\ell}\right)$, multiples of $\pi$ do not contribute and the scattering is determined by $\delta_{\ell}$, which is relatively small in those metals and semiconductors where the pseudopotential approach is valia.

A pseudopotential is a relatively weak potential, having the same effective scattering power as the strong real potential. In general it is a complicated non local operator. A local operator $\mathrm{F}$ is a function of $r$ only and $\mathrm{F} \phi$ denotes $\mathrm{F}(\underline{x}) \phi(\underline{x})$. A non local operator depends on two variables, $F\left(\underline{r}, \underline{r}^{\prime}\right)$, and $\mathrm{F} \phi$ is given by

$$
F \phi=\int F\left(\underline{r}, \underline{r}^{\prime}\right) \phi\left(\underline{x}^{\prime}\right) d \underline{r}^{\prime} .
$$

When setting up the theory it is assumed that a clear physical distinction can be made between the conduction electrons of the system and the core electrons. It is desirable that the core states be strongly localized in the vicinity of the ions and that the ionic cores be small so that the outermost states do not significantly overlap with the outermost states of the nearest neighbours. If this last condition does not hold the outermost core states may participate in the conduction band to some extent, causing problems of band 
overlap and hybridization. This situation exists in the noble metals and it is uncextain to what extent pseudopotential theory may be used in these cases. This problem also exists to a lesser extent in mercury.

In the case of a metal the conduction band Bloch states satisfy the Schrödinger equation.

$$
H\left|\psi_{\underline{k}}>=E_{k}\right| \psi_{k}>
$$

where the Hamiltonian $H$ given by $H=T+V(\underline{x})$; $T$ being the kinetic energy operator and $V(\underline{r})$ the real crystal potential. The solution of equation (2.2) requires the use of a convenient complete set of functions. The most simple complete set to use is the set of plane waves, but $\psi_{\mathrm{k}}$ possesses several radial nodes and a good representation would require a large number of terms in the expansion and the resulting secular equation would be extremely large and difficult to handle.

Herring (1940) recognized that the problem could be simplified if the expansion were made in terms of plane waves that had already been orthogonalized to the core states of the system. If a plane wave is represented by the ket:$|\underline{k}\rangle=\frac{1}{\sqrt{V}} e^{i k \cdot \underline{x}}$, where $V$ is the volume of the crystal and a core state represented by $|\alpha\rangle \equiv \psi_{\alpha}(r)$. Then if $P$ represents the operator introduced by Pick and Sarma (1964), which projects any function on to the subspace of the core states. 


$$
P=\sum_{\alpha}|\alpha><\alpha|
$$

In terms of this operator an orthogonalized plane wave takes the form

$$
|O P W\rangle_{\underline{k}}=(1-P) \mid \underline{k}>
$$

It has been found that good convergence can often be obtained by using a linear combination of a small number of OPW's.

$$
\left|\psi_{\underline{k}}\right\rangle=\underset{\underline{q}}{\Sigma} a_{\underline{q}}(\underline{k})(1-p) \mid \underline{k}+\underline{q}>;
$$

Or, more generally

$$
\left|\psi_{\underline{\underline{k}}}\right\rangle=(1-P)\left|\phi_{\underline{\underline{k}}}\right\rangle
$$

The pseudo-wave function $\phi_{\underline{k}}(\underline{r})$ is equal to the true wave function $\psi_{\underline{k}}(\underline{r})$ outside the ionic cores but inside the cores $\phi_{\underline{k}}(\underline{r})$ is slowly varying and has no radial nodes. Equation (2.3) does not completely define $\phi_{\underline{k}}(\underline{\underline{r}})$ it being undefined to the extent that any linear combination of core states can be added to it.

$$
\text { Substituting } \psi_{\underline{k}} \text { from equation (2.3) into equation }
$$

$$
H(1-P)\left|\phi_{k}>=E_{k}(I-P)\right| \phi_{k}>
$$

Phillips and kleinman (1959) noted that the equation can be rearranged to give

$$
\left[H+\left(E_{k}-H\right) P\right]\left|\phi_{k}>=E_{k}\right| \phi_{k}>
$$


In which the pseudo-Hamiltonian

$$
H_{p}=H+\left(E_{k}-H\right) P,
$$

can be written as

$$
H_{p}=T+W_{p} \cdot
$$

where the pseudopotential operator $W_{p}$ is given by

$$
W_{p}=V(\underline{r})+\left(E_{k}-H\right) P .
$$

In this case

$$
W_{p}=V(\underline{x})+\sum_{\alpha}\left(E_{k}-E_{\alpha}\right)|\alpha><\alpha|
$$

Where $E_{\alpha}$ is the energy of the $\alpha^{\text {th }}$ core state which satisfies the equation.

$$
H|\alpha\rangle=E_{\alpha}|\alpha\rangle
$$

The pseudo--Hamiltonian that has been introduced is only one of many possibilities. Austin, Heine and Sham (1962) have shown that the general class of pseudo-Hamiltonians

$$
\mathrm{H}_{\mathrm{p}}=H+\sum_{\alpha}|\alpha\rangle\langle\underline{\mathrm{f}}(\underline{\underline{r}}, \alpha)|,
$$

in which $f(\underline{r}, \alpha)$ is an arbitrary function of position and core index $\alpha$, all have exactly the same eigenvalues $E_{K}$ in the conduction band as the original Hamiltonian.

1. Solution of the Pseudopotential Eigenvalue Problem

The equation to be solved is

$$
\left.\left(H+\sum_{\alpha_{k}}\left(E_{k}-E_{\alpha_{k}}\right)\left|\alpha_{k}><\alpha_{k}\right|\right)\left|\phi_{k}>=E_{k}\right| \phi_{k}\right\rangle
$$


in which the core states $\left|\alpha_{k}\right\rangle$ can be written in the tight binding approximation.

$$
|\alpha t, \underline{k}\rangle=\frac{1}{\sqrt{\mathbb{N}}} \sum_{j} \exp \left(\underline{i \underline{k} \cdot} \cdot R_{\underline{j \alpha}}\right) \phi_{\alpha t}\left(\underline{x-R_{j \alpha}}\right)
$$

where $N=$ the number of unit cells in the crystal.

$$
j=\text { the unit cell index. }
$$

at refers to the $t^{\text {th }}$ level corresponding to the atom at $r_{\alpha}$ in the unit cell.

$$
\text { Since } \left.\phi_{\underline{k}} \underline{\underline{r}}\right) \text { has wave number } \underline{k} \text {, then by Bloch's }
$$

theorem,

$$
\left.\phi_{\underline{k}} \underline{\underline{r}}\right)=e^{i \underline{k} \cdot \underline{\underline{r}}} a_{\underline{k}} \underline{(\underline{r})}
$$

where $a_{k}(\underline{r})$ is a function having the exact periodicity of the lattice. This means that $\phi_{\underline{k}} \underline{\underline{x}}$ ) can be completely expanded. in terms of the subset of plane waves.

$$
\left|\phi_{\underline{\mathrm{k}}} \underline{(\underline{r})}>=\sum_{\underline{G}} A_{\underline{G}}\right| \underline{k}+\underline{G}>.
$$

where $G$ is a reciprocal lattice vector multiplied by $2 \pi$. The matrix elements of the pseudo-Hamiltonian will all be of the form $\left\langle\underline{k}+\underline{G}_{1}\left|H_{p}\right| \underline{k}+\underline{G}_{2}>\right.$.

2. Calculation of the Matrix Elements

The general matrix elements $\left\langle\underline{k}_{-} \underline{G}_{1}\left|H_{p}\right| \underline{k}+G_{2}\right\rangle$ is equal to a sum of terms,

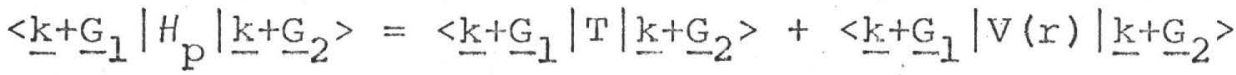

$$
\begin{aligned}
& +\sum_{\alpha_{K}}\left(E_{K}-E_{\alpha K}\right)<\underline{K}+\underline{G}_{I}\left|\alpha_{K}><\alpha_{K}\right| \underline{k}+\underline{G}_{2}>
\end{aligned}
$$


Each term can be evaluated separately,

$\left\langle\underline{k}+\underline{G}_{1}|\mathrm{~T}| \underline{k}+\underline{G}_{2}\right\rangle=\left\langle\underline{k}+\underline{G}_{1}\left|-\frac{\varkappa^{2}}{2 m} \nabla^{2}\right| \mathrm{k}+G_{2}\right\rangle=-\frac{\hbar^{2}}{2 m}\left|\underline{k}+\underline{G}_{2}\right|^{2} \delta_{G_{1}}, \underline{G}_{2}$

The real potential $\mathrm{V}(\underline{\underline{r}})$ has the periodicity of the real lattice, it can be expanded in a Fourier series.

giving

$$
V(\underline{\underline{r}})=\sum_{\underline{G}} V(\underline{G}) \exp (i \underline{G} \cdot \underline{r})
$$

$$
\left\langle\underline{k}+\underline{G}_{I}|V(\underline{r})| \underline{k}+G_{2}\right\rangle=V\left(\underline{G}_{I}-\underline{G}_{2}\right)
$$

where the Fourier components $\mathrm{V}(\mathrm{G})$ are equal to

$$
V(G)=\frac{l}{V} \int V(\underline{r}) \exp (-\underline{\underline{G}} \cdot \underline{r}) d \underline{r} ;
$$

the $\mathrm{V}$ in the denominator referring to the crystal volume.

It is sometimes useful to treat $V(\underline{r})$ as a superposition of atomic sources.

$$
V(\underline{r})=\sum_{j, \alpha} U_{p, \alpha}\left(\underline{r}-\underline{R}_{j \alpha}\right)
$$

Giving

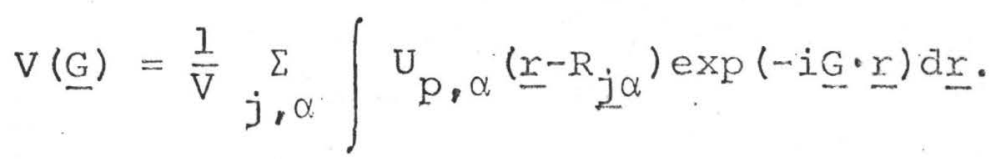

which can be rewritten

$$
V(\underline{G})=\frac{J}{V} \sum_{j, \alpha} \int U_{p, \alpha}\left(\underline{r}-R_{j \alpha}\right) \exp \left(-i \underline{\underline{C}} \cdot\left(\underline{r}-R_{j}\right) \exp \left(-\underline{i} \cdot \underline{R}_{j \alpha}\right) d\left(\underline{r}-R_{j \alpha}\right)\right.
$$

or,

$$
V(\underline{G})=\frac{1}{\bar{V}} \sum_{j, \alpha} \exp \left(-\underline{i} \underline{G} \cdot R_{j \alpha}\right) \int U_{p \alpha}(\underline{\underline{r}}) \exp (-\underline{i} \underline{\underline{G}} \cdot \underline{\underline{r}}) \mathrm{d} \underline{\underline{r}}
$$

Defining the atomic form factor by 


$$
\begin{gathered}
U_{p \alpha}(\underline{G})=\int U_{p \alpha}(\underline{r}) \exp (-i \underline{\underline{G}} \cdot \underline{\underline{r}}) d \underline{\underline{r}} \cdot \\
V(\underline{G})=\frac{1}{V} \sum_{\alpha, j} \exp \left(-\underline{i} \underline{G}^{\bullet} R_{j}\right) \exp \left(-i \underline{G} \cdot \underline{x}_{\alpha}\right) U_{p \alpha}(\underline{G})
\end{gathered}
$$

Therefore,

$$
V(\underline{G})=\frac{N}{V} \sum_{\alpha} \exp \left(-i \underline{G} \cdot \underline{R}_{\alpha}\right) U_{p(\alpha}(G) .
$$

If there is only one type of atom present

$$
V(\underline{G})=\frac{1}{\Omega}\left(\sum \exp \left(-i \underline{G} \cdot R_{\alpha}\right)\right) U_{p}(\underline{G})
$$

$\Omega$ is the volume of the unit cell. The unit cell structure factor is defined by

$$
S(\underline{G})=\sum_{\alpha}^{\sum} \exp \left(-\underline{i G} \cdot R_{\alpha}\right) .
$$

Giving

$$
V(\underline{G})=\frac{1}{\Omega} S(\underline{G}) U_{p}(\underline{G})
$$

To calculate the last term in equation (2.9) it is easiest to take the various factors separately

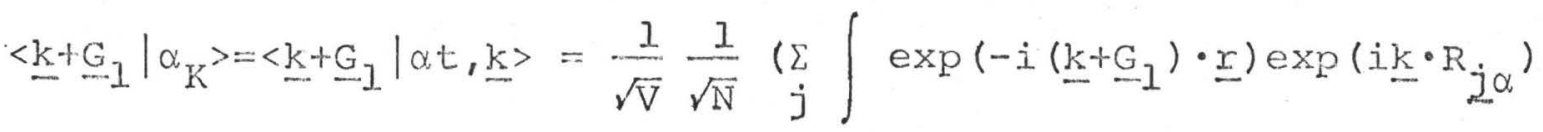

$$
\begin{aligned}
& \left.\times \phi_{\alpha t}\left(\underline{r}-R_{j \alpha}\right) a^{3} \underline{r}\right)
\end{aligned}
$$

so that,

$$
\begin{aligned}
& \left\langle\underline{k}+\underline{G}_{1} \mid \alpha t, \underline{k}\right\rangle=\frac{1}{\sqrt{N}} \frac{1}{\sqrt{N}}\left(\int \exp \left(-i\left(\underline{k}+\underline{G}_{1}\right) \cdot\left(\underline{r}-R_{\underline{j} \alpha}\right) \phi_{\alpha t}\left(\underline{r}-R_{j \alpha}\right) d^{3}\left(\underline{r}-R_{j \alpha}\right)\right)\right. \\
& \times\left(\sum_{j} \exp \left(-i\left(k+G_{1}\right) \cdot R_{j \alpha}+i \underline{k} \cdot R_{j \alpha}\right)\right.
\end{aligned}
$$


Therefore

$$
\begin{aligned}
& \left\langle\underline{k+G_{1}} \mid \alpha t, \underline{k}\right\rangle=\frac{I}{\sqrt{V}} \frac{1}{\sqrt{N}}\left(\int \exp \left(-i\left(\underline{k}+\underline{G}_{1}\right) \cdot \underline{r}\right) \phi_{\alpha t}(\underline{r}) d^{3} \underline{\underline{r}}\right) \\
& \times \sum_{j} \exp \left(-\underline{i} \underline{G}_{I} \cdot R_{j \alpha}\right)
\end{aligned}
$$

The sum $\sum_{j} \exp \left(-i G_{I} \cdot R_{j \alpha}\right)=\exp \left(-i G_{j} \cdot R_{-\alpha}\right) \sum_{j} \exp \left(-i G_{1} \cdot R_{j}\right)$,

but

$$
\exp \left(-i G_{1} \cdot R_{j}\right)=1 .
$$

So that

$$
\sum_{j} \exp \left(-i \underline{G}_{I} \cdot R_{j \alpha}\right)=N \exp \left(-i G_{I} \cdot R_{\alpha}\right)
$$

Substituting back into equation (2.13)

$\left\langle\underline{\mathrm{k}}+\underline{G}_{I} \mid \alpha t, \underline{\mathrm{k}}\right\rangle=\sqrt{\frac{\mathrm{N}}{\mathrm{V}}} \exp \left(-i \underline{G}_{I} \cdot R_{\alpha}\right) \int \exp \left(-i\left(\underline{k}+\underline{G}_{I}\right) \cdot x\right) \times \phi_{\alpha t}(\underline{\underline{r}}) \mathrm{a}^{3} \underline{\underline{r}}$.

This means that

$$
\begin{gathered}
\left\langle\underline{k+G_{I}} \mid \alpha t, \underline{k}\right\rangle\left\langle\alpha, \underline{\underline{k}} \mid \underline{k}+\underline{G}_{2}\right\rangle=\frac{N}{\bar{V}} \exp \left(-i\left(\underline{G}_{-}-\underline{G}_{2}\right) \cdot R_{\alpha}\right) \\
\times \int \exp \left(-i\left(\underline{k}+\underline{G}_{I}\right) \cdot \underline{r}\right) \phi_{\alpha t}(\underline{r}) d^{3} \underline{r} \times \int \exp \left(i\left(\underline{k}+\underline{G}_{2}\right) \cdot r\right) \phi_{\alpha t}^{*}(\underline{r}) \alpha^{3} \underline{r} .
\end{gathered}
$$

Summing over the atoms in the unit cell $\alpha$

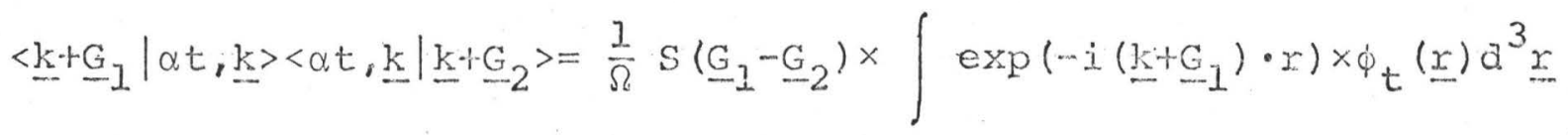

$$
\begin{aligned}
& \times \int \exp \left(i\left(\underline{\underline{k}}+\underline{G}_{2}\right) \cdot \underline{\underline{r}}\right) \phi_{t}^{*}(\underline{r}) \dot{d}^{3} \underline{\underline{r}} \text {. }
\end{aligned}
$$

In this way equation (2.9) simplifies to the expression $\left\langle\underline{k}+\underline{G}_{1}\left|H_{p}\right| \underline{k}+\underline{G}_{2}\right\rangle=\frac{\pi^{2}}{2 m}\left|\underline{k}+\underline{G}_{2}\right|^{2} \delta_{G_{1}, G_{2}}+\frac{l}{\Omega} S\left(\underline{G}_{2}-G_{2}\right) U_{p}\left(G_{1}-G_{2}\right)$ 


$$
\begin{aligned}
& +\frac{1}{\Omega} S\left(\underline{G}_{1}-\underline{G}_{2}\right) \sum\left[\int \exp \left(-i\left(\underline{k}+\underline{G}_{1}\right) \cdot r\right) \phi_{t}(\underline{r}) d^{3} \underline{r}\right. \\
& \left.\times \int \exp \left(i\left(\underline{k}_{t}+\underline{G}_{2}\right) \cdot \underline{r}\right) \phi_{t}^{*}(\underline{r}) d^{3} \underline{r}\right] \times\left(E_{k}-E_{t}\right)
\end{aligned}
$$

To simplify the last term in equation (2.15), take $\phi_{t}(\underline{r})$ to be given in the standard form as computed and recorded by Herman and Skillman (1965).

$$
\phi_{t}(\underline{r})=\frac{P_{n \ell}(r)}{r} Y_{\ell m}(\theta, \phi)=\phi_{n \ell m}(\underline{r}) .
$$

The radial wave function $P_{n l}(r)$ is necessarily zero at $r=0$. Also by normalization $\int_{0}^{\infty} \mathrm{P}_{n l} 2(r) \mathrm{dr}=1$.

To evaluate expressions like $\sum_{n, l, m}\left(E_{K}-E_{n \ell m}\right) \int e^{-i\left(\underline{k}_{1} \cdot \underline{r}\right)} \phi_{n \ell m}(\underline{r}) d \underline{r} \times \int e^{i \underline{k}_{2} \cdot \underline{r}} \phi_{n \ell m}^{*}(\underline{r}) d \underline{r}$, it is possible to sum over the magnetic quantum number $m$ first, assuming that $E_{n \ell m}$ is independent of $\mathrm{m}$.

$$
\begin{aligned}
& \sum_{m}\left(E_{K}-E_{n \ell m}\right) \int e^{-i \underline{k} l \cdot \underline{r}} \frac{1}{r} P_{n l}(r) Y_{\ell m}(\theta, \phi) r^{2} d r d \Omega \\
& \times \int e^{i \underline{k}_{2} \cdot \underline{r}} \frac{1}{r} P_{n \ell}(r) Y_{\ell m}^{*}(\theta, \phi) r^{2} d r d \Omega \\
& =\left(E_{K}-E_{n l}\right) \sum_{m}\left(\int e^{-\underline{k}-1 \cdot \underline{r}} P_{n \ell}(r) r Y_{\ell m}(\theta, \phi) d \Omega d r\right) \\
& \times\left(\int e^{i \underline{k}{ }_{2} \cdot \underline{r}} P_{n \ell}(r) r Y_{\ell m}^{*}(\theta, \phi) d \Omega d r\right)
\end{aligned}
$$

The integrals can be transformed by making use of the expansion, 
$e^{i \underline{k} \cdot \underline{r}}=4 \pi \sum_{\ell^{\prime}=0}^{\infty} \sum_{m^{\prime}=-\ell^{\prime}}^{\ell^{\prime}}(i)^{\ell^{\prime}} j_{\ell^{\prime}}(k r) Y_{\ell^{\prime} m^{\prime}}\left(\theta_{k}, \phi_{k}\right) Y_{\ell m}\left(\theta_{r^{\prime}} \phi_{r}\right)$

$\left(\theta_{k}, \phi_{k}\right),\left(\theta_{r}, \phi_{r}\right)$ are the spherical polar angles of the vectors $\underline{k}$ and $\underline{r}$. If the expansion for $\exp (\underline{i k} \cdot \underline{r})$ is substituted into each integral, and the angular parts are integrated immediately by making use of the orthonormality of the spherical harmonics

$$
\int Y_{\ell m}{ }^{*} Y_{\ell ' m}, d \Omega=\delta_{m m} \delta_{\ell \ell}
$$

Expression (2.16) now simplifies to

$$
\begin{aligned}
16 \pi^{2}\left(E_{k}-E_{n \ell}\right) & \sum(-i)^{\ell} \int_{0}^{\infty} j_{\ell}\left(k_{1} r\right) Y_{\ell m}\left(\theta_{k 1}, \phi_{k 1}\right) r P_{n \ell}(r) d r \\
& \times(i)^{\ell} \int_{0}^{\infty} j_{\ell}\left(k_{2} r\right) Y_{\ell m}{ }^{*}\left(\theta_{k 2}, \phi_{k 2}\right) r P_{n \ell}(r) d r
\end{aligned}
$$

which is equal to

$$
\begin{aligned}
& 16 \pi^{2}\left(E_{k}-E_{n \ell}\right)\left[\sum_{m} Y_{\ell m}\left(\theta_{k 1}, \phi_{k 1}\right) Y_{\ell m}{ }^{*}\left(\theta_{k 2}, \phi_{k 2}\right)\right] \\
& \times \int j_{\ell}\left(k_{1} r\right) r P_{n \ell}(r) d r \times \int j_{\ell}\left(k_{2} r\right) r P_{n \ell}(r) d r .
\end{aligned}
$$

But from the addition theorem of spherical harmonics

$$
\sum_{\mathrm{m}=-\ell}^{\mathrm{m}=+\ell} \mathrm{Y}_{\ell \mathrm{m}}{ }^{*}\left(\theta_{1}, \phi_{1}\right) \mathrm{Y}_{\ell \mathrm{m}}\left(\theta_{2}, \phi_{2}\right)=\frac{(2 \ell+1))}{4 \pi} \mathrm{P}_{\ell}(\cos \alpha)
$$

where $\alpha$ is the angle between the directioms $\left(\theta_{1}, \phi_{1}\right)$ and $\left(\theta_{2}, \phi_{2}\right)$. $P_{\ell}$ is the Legendre polynomial of order $\ell$. Finally expression (2.16) reduces to 


$$
\begin{gathered}
4 \pi(2 \ell+1)\left(E_{k}-E_{n \ell}\right) P_{\ell}\left(\cos \left(\underline{k}_{1}, \underline{k}_{2}\right)\right) \times \int j_{\ell}\left(k_{1} r\right) r P_{n \ell}(r) d r \\
\times \int j_{\ell}\left(k_{2} r\right) r P_{n \ell}(r) d r
\end{gathered}
$$

the matrix element being given by

$$
\begin{aligned}
& \left\langle\underline{k}+\underline{G}_{1}\left|H_{p}\right| \underline{k}+\underline{G}_{2}\right\rangle=\frac{\hbar^{2}}{2 m}\left|k+G_{1}\right|^{2} \delta_{G_{1}, G_{2}}+\frac{1}{\Omega} U\left(\underline{G}_{1}-\underline{G}_{2}\right) \\
& +\frac{4 \pi}{\Omega} \sum_{n \ell}\left(E_{k}-E_{n \ell}\right)(2 \ell+1) P_{\ell}\left(\cos \left(\underline{k}_{+} \underline{G}_{I}, \underline{k}+\underline{G}_{2}\right)\right) \times \int j_{\ell}\left(\left|\underline{k}+\underline{G}_{1}\right| r\right) r P_{n \ell}(r) d r \\
& \times \int j_{\ell}\left(\left|\underline{k}+\underline{G}_{2}\right| r\right) r P_{n \ell}(r) d r
\end{aligned}
$$

3. Conversion to Atomic Units

It is convenient in energy band calculations to use atomic units. One atomic unit of length $=\frac{\hbar^{2}}{\mathrm{me}^{2}}=0.52917$ Angstrom units. To convert formulae to the atomic system put $e=1, m=1, K=1$. This will give energies in atomic units, but it is customary to express energies in Rydbergs.

1 atomic unit of energy $=2$ Rydbergs.

In this system of units equation (2.22) can be rewritten as

$$
\begin{gathered}
\left\langle\underline{k}+\underline{G}_{1}\left|H_{p}\right| \underline{k}+\underline{G}_{2}>=\left|\underline{k}_{\underline{G}}\right|^{2} \delta_{G_{1}, G_{2}}+\frac{1}{2} U\left(\underline{G}_{1}-\underline{G}_{2}\right)\right. \\
+\frac{4 \pi}{\Omega} \sum_{n, l}\left(E_{k}-E_{n \ell}\right)(2 \ell+1) P_{\ell}\left(\cos \left(k+G_{1}, k+G_{2}\right)\right) * \int j_{\ell}\left(\left|k+G_{1}\right| r\right) r P_{n \ell}(r) d r \\
\times \int j_{\ell}\left(\left|\underline{k}+\underline{G}_{2}\right| r\right) r P_{n \ell}(r) d r
\end{gathered}
$$


where all energies are understood to be in terms of Rydbergs.

4. The Evaluation of Integrals Containing Spherical Bessel. Functions

When calculating integrals containing spherical Bessel functions it is possible to make use of the formulae

$$
j_{\ell}(\rho)=\left(\frac{\pi}{2 \rho}\right)^{\frac{1}{2}} J_{\ell+\frac{1}{2}}(\rho)
$$

But the half integral regular Bessel functions are given by the simple trigonometric formulae

$$
\begin{aligned}
& \mathrm{J}_{\frac{\pi}{2}}(\rho)=\sin \rho \times\left(\frac{2}{\pi \rho}\right)^{\frac{1}{2}} \\
& \mathrm{~J}_{\frac{3}{2}}(\rho)=\left(\frac{\sin \rho}{\rho}-\cos \rho\right)\left(\frac{2}{\pi \rho}\right)^{\frac{1}{2}} \\
& \mathrm{~J}_{\frac{5}{2}}(\rho)=\left(\left(\frac{3}{\rho^{2}}-1\right) \sin \rho-\frac{3}{\rho} \cos \rho\right)\left(\frac{2}{\pi \rho}\right)^{\frac{1}{2}} \\
& \mathrm{~J}_{\frac{7}{2}}(\rho)=\left(\left(\frac{15}{\rho^{3}}-\frac{6}{\rho}\right) \sin \rho-\left(\frac{15}{\rho^{2}}-1\right) \cos \rho\right) \times\left(\frac{2}{\pi \rho}\right)^{\frac{1}{2}} .
\end{aligned}
$$

Rearranging terms so that the same powers of $p$ are grouped together.

$$
\begin{aligned}
& j_{0}(\rho)=\frac{\sin \rho}{\rho} \\
& j_{1}(\rho)=\frac{1}{\rho}\left(\frac{\sin \rho}{\rho}-\cos \rho\right) \\
& j_{2}(\rho)=\frac{1}{\rho}\left(\frac{3}{\rho}(\sin \rho-\rho \cos \rho)-\sin \rho\right) \\
& j_{3}(\rho)=\frac{1}{\rho}\left(\frac{15}{\rho}(\sin \rho-\rho \cos \rho)-\frac{1}{\rho}(6 \sin -\rho \cos \rho)\right) .
\end{aligned}
$$

The variation of $j_{l}(\rho)$ as $\rho \rightarrow 0$, is given by

$$
j_{\ell}(\rho) \underset{p \rightarrow 0}{\sim} \frac{\rho^{\ell}}{(2 \ell+1) ! !}\left[1-\frac{\rho^{2}}{2(2 \ell+3)}+\ldots \ldots\right]
$$


5. Other Types of Pseudopotential

The pseudopotential introduced in equation (2.7)

$$
W_{p}=V(\underline{r})+\sum_{\alpha}\left(E_{k}-E_{\alpha}\right)|\alpha><\alpha|
$$

is a non local operator. A local operator is easier to use because it has matrix elements $\left\langle\underline{k}+\underline{q}\left|W_{p}\right| \underline{k}\right\rangle$ which depend upon g only. Whereas in the case of the a non local operator it depends upon $(k+g)$ and $\underline{k}$.

As was noted earlier Austin, Heine and Sham (1962) have shown that the pseudopotential of equation (2.26) is only one of a more general class.

$$
W_{p}=V(\underline{r})+\sum_{\alpha}|\alpha><E(\underline{r}, \alpha)|
$$

The general pseudopotential. $W_{p}$ is an operator not a function, it is incorrect to write it in the form $W_{p}(\underline{x})$. It can be completely specified in terms of its action on a set of plane waves.

$$
\mathrm{W}_{\mathrm{p}}|\underline{\mathrm{k}}\rangle=\mathrm{V}|\underline{\mathrm{k}}\rangle+\sum_{\alpha} \mathrm{f}(\mathrm{k}, \alpha)\langle\alpha|k>| \alpha\rangle
$$

where

$$
\underline{E}(\underline{\underline{r}}, \alpha)=\sum_{k} \mathrm{f}^{*}(k, \alpha)\langle\mathrm{k} \mid \alpha\rangle|\mathrm{k}\rangle,
$$

defines $f(k, \alpha)$. The general pseudopotential operator can be completely specified in terms of its matrix elements $<\underline{k}+\underline{q}\left|w_{p}\right| \underline{k}>$. These matrix elements are called form factors.

An approximation that is often used is to restrict the set of form factors to those cases in which the magnitude 
of $\underline{k}$ and $\underline{k}+\underline{q}$ are both equal to the single value $k_{F}$, where $k_{F}$ is equal to the radius of the free electron sphere. For a given $q$ with magnitude less than $2 k_{F}$ the angle between $\underline{k}$ and $\underline{k}+q$ is fixed and a single value of $\left\langle\underline{k}+\underline{q}\left|w_{p}\right| \underline{k}\right\rangle$ obtains. This restricted set of form factors which are referred to as the OPW form factors have the property of a local operator. For this reason the approximation is often made that the pseudopotential to be used should be a local operator. Whether or not this is a good approximation will depend upon the element being investigated and also upon which property is being considered.

The reason the pseudopotential operator is weak is not quite obvious but there is one formulation in which it is easier to demonstrate that $W_{p}$ is small. Following Austin, Heine and Sham one particular form of $W_{p}$ that is admissible is

$$
\mathrm{W}_{\mathrm{p}}=(1-\mathrm{P}) \mathrm{V} \text {. }
$$

Giving

$$
\mathrm{w}_{\mathrm{p}}|\phi\rangle=\mathrm{V}|\phi\rangle-\Sigma|\alpha\rangle\langle\alpha \mathrm{V} \mid \phi\rangle
$$

Inside the ionic cores the set of core states will form almost a complete set so far as the real potential is concerned, so that $W_{p}$ will be almost exactly zero inside a certain radius and outside that radius it can be approximated by the coulomb potential of the ion.

This approach leads to the model potential of Abarenkov 
and Heine (1965). In this model there is a simple square well of depth A inside some model radius $\mathrm{R}_{\mathrm{M}}$ and the appropriate coulomb potential outside.

$$
\begin{array}{ll}
W_{p}=-\sum_{\ell} \mathbb{A}_{\ell}(\varepsilon) P_{\ell} & \left(x<R_{M}\right) \\
W_{p}=-\frac{z}{r} & \left(x>R_{M}\right)
\end{array}
$$

In these expressions $\mathrm{Z}$ is the coulomb charge of the ion, $\mathrm{P}_{\ell}$ is a projection operator that picks out the $e^{\text {th }}$ angular momentum components from any function upon which $W_{p}$ acts. The parameters $\mathrm{A}_{\ell}$ are adjustable so that (2.30) reproduces exactly the spectroscopically observed energy levels of one electron added to the ion. A $A_{\ell}$ also has to depend slightly on $\varepsilon$ to give exactly the correct energy sequence.

It is also necessary to include the effects of the conduction electrons. The most important of these effects are correlations, exchange and screening. Correlation is related to the fact that each electron in the system exerts a coulomb repulsion upon the other electrons thus surrounding itself with a correlation hole. Exchange is due to the Pauli principle in that two electrons of the same spin cannot occupy the same position simultaneously, so there will also be an exchange hole surrounding each electron so far as electrons of the same spin are concerned.

Screening is a very important effect that can be treated 
approximately by using the Hartree dielectric function for free electrons. In this approximation the unscreened matrix element $\left\langle\mathrm{k}+\mathrm{q}\left|\mathrm{w}_{\mathrm{p}}{ }^{\circ}\right| \mathrm{k}\right\rangle$ is divided by $\varepsilon(q)$ to give the screened matrix element $\left\langle k+q\left|w_{p}\right| k>\right.$.

$$
\left\langle\mathrm{k}+\mathrm{q}\left|\mathrm{w}_{\mathrm{p}}\right| \mathrm{k}\right\rangle=\left\langle\mathrm{k}+\mathrm{q}\left|\mathrm{w}_{\mathrm{p}}{ }^{\circ}\right| \mathrm{k}\right\rangle / \varepsilon(\mathrm{q})
$$

$\varepsilon(q)$ is the Hartree dielectric function

$$
\begin{gathered}
\varepsilon(q)=1+\frac{m e^{2}}{2 \pi k_{F} \hbar^{2} \eta^{2}}\left(\frac{1-\eta^{2}}{2 \eta} \log _{e}\left|\frac{1+n}{1-\eta}\right|+1\right) \\
n=\frac{q}{2 k_{F}} .
\end{gathered}
$$

The Abarenkov-Heine pseudopotential is not a pseudopotential in the sense of Austin, Heine, and Sham, which is applied to a mathematical class involving projections on to the core states. The term pseudopotential has been extended to cover any model potential which has the same phase shifts as the real potential but with the multiples of $\pi$ removed. Preferably the model potential should be as weak as possible.

6. The Choice of a Suitable Approximation

The pseudo-Hamiltonian equation introduced in equation $(2.7)$

$$
\left(H+\sum_{\alpha}\left(E_{k}-E_{\alpha}\right)|\alpha\rangle\langle\alpha|\right)\left|\phi_{k}\right\rangle=E_{k}\left|\phi_{k}\right\rangle,
$$

is really an exact expression. If the core energies $E_{\alpha}$ and the core wave functions were known exactly, then it would be 
possible to get an exact solution once the Fourier components $\mathrm{V}(\mathrm{G})$ of the real potential had been found.

One problem that axises from using equation (2.7) is that if it is used to calculate the energy bands, the equation will have to be solved iteratively because it has an energy dependence on both sides. To facilitace the calculation it is possible to put the coefficients $\left(E_{k_{k}}-E_{\alpha}\right)$ arbitrarily equal to constants $\left(E_{F}-E_{\alpha}\right)$, where $E_{F}$ is the Fermi energy. This will give energy bands that are correct around the Fermi energy but which will systematically deviate from the correct values at other energies.

As most pseudopotential calculations make use of adjustable parameters that have been determined by an empirical fit to the measured dimensions of the Fermi surface, it is intrinsic that energy bands calculated by the pseudopotential will be most correct near the Fermi enexgy and will be increasingly in error the farther away from the Fermi energy.

Kimball, Stark, and Muellex (1967) have made use of experimental de Haas van Alphen periods and the magneto-acoustic calipers of magnesium to yield a pseudopotential which could then be used to calculate the rest of the Fermi surface. They first tried a purely local pseudopotential, but although this worked fairly well they obtained substantially better agreement by making use of the non-local pseudopotential (2.7). In the case of magnesium the problem is fairly well defined in the 
sense that the only core states that have to be considered are the 1s, $2 \mathrm{~s}$, and $2 \mathrm{p}$ levels. Relativistic effects are small and the pseudopotential that is finally determined involves the Fourier components of the real potential V(G). Mercury is a heavy element, atomic number $z=80$, and it should be expected that relativistic effects are important. There is also some experimental evidence, Wilson and Rice (1966), and theoretical evidence, Keeton and Loucks (1966), that the top of the $5 d$ bands cuts across the bottom of the conduction bands. In this respect mercury resembles the noble metals to some extent. It is not immediately obvious that the pseudopotential concept can be applied to the noble metals and it appears that mercury is a borderline case. If a partial wave analysis were applied to those conduction band states that overlap with or are just above the a bands there should be a strong scattering resonance for the $l=2$, partial wave. But if the Fermi energy is sufficiently far above the d bands then some sort of pseudopotential approximation may suffice for Fermi surface calculations.

In mercury the occupied core states are $1 \mathrm{~s}, 2 \mathrm{~s}, 2 \mathrm{p}$, $3 s, 3 p, 3 d, 4 s, 4 p, 4 d, 4 f, 5 s, 5 p, 5 d$. The use of the pseudopotential of equation (2.7)

$$
\mathrm{W}_{\mathrm{p}}=\mathrm{V}(\underline{\underline{r}})+\sum_{\alpha}\left(\mathrm{E}_{\mathrm{K}}-\mathrm{E}_{\alpha}\right)|\alpha><\alpha|
$$

involves the calculation of matrix elements of the form 


$$
\left\langle\underline{k}_{1}\left|w_{p}\right| \underline{k}_{2}\right\rangle=V\left(\underline{k}_{1}-\underline{k}_{2}\right)+\sum\left(E_{k}-E_{\alpha}\right)<k_{1}|\alpha\rangle\left\langle\alpha \mid k_{2}\right\rangle
$$

It follows from equation (2.23) that the matrix elements involving s like core states are independent of the angle between $\underline{k}_{1}$ and $\underline{k}_{2}$. The core states of higher angular momentum will depend on this angle through the Legendre polynomial $P_{\ell}\left(\cos \left(k_{1}, k_{2}\right)\right)$. If it can be demonstrated that for the values of $\underline{k}_{1}$ and $\underline{k}_{2}$ of interest, values having $|\underline{k}| \leq 2 k_{F}$ approximately, that the $p$, $d$, and $f$ core states yield terms that are very small whilst the s states yield terms that are not so small but are effectively independent of $\left|\underline{k}_{1}\right|$ and $\left|\underline{k}_{2}\right|$, then it may be possible to group the inner core states with the real potential to give the model pseudopotential.

$$
\begin{aligned}
& W_{p}=W_{L O C}\left(\underline{k}_{1}-\underline{k}_{2}\right)+\sum_{\alpha} \quad\left(E_{k}-E_{\alpha}\right)|\alpha><\alpha| . \\
& \text { summing over } \\
& \text { outer core states }
\end{aligned}
$$

in which $\mathrm{W}_{\text {LOC }}$ is a local operator.

The next approximation that can be made is to set $\left(E_{k}-E_{\alpha}\right)$ equal to the constant $\left(E_{F}-E_{\alpha}\right)$ if only properties of the Fermi surface are being considered.

As there is a strong relativistic interaction in heavy elements the energies of the core levels relative to the conduction band will not necessarily be the same as in the calculations of Herman and Skillman relating to the atom. The crystal field can also significantly alter the relative energies of the outer bands. 
With such a model pseudopotential as (2.33) the disposable parameters are $W_{L O C}(G)$ and $\left(E_{F}-E_{\alpha}\right)$. This can easily lead to an inconveniently large number of parameters if too many outer core states are explicitly considered. The problems of using a large number of disposable parameters are that if a search is carried out in parameter space to find those regions which give good agreement to experimental data the search can be extremely costly in computer time. It may also be possible to find several regions which give equally good agreement to the available experimental data. Increasing the number of disposable parameters may eventually yield a better fit to more experimental data, but the resulting pseudopotential may also be physically meaningless because the use of purely arbitrary parameters could also provide an improved fit.

A good pseudopotential is an approximation that is simple in form and easy to use, it should incorporate those terms that are physically significant, and should ideally be a good approximate representation of the interaction between the system of conduction electrons and the ions.

An example of a non-local pseudopotential has been given by Stark and Falicov (1967) for the elements zinc and cadmium . In their model which includes spin-orbit coupling the matrix elements are given by

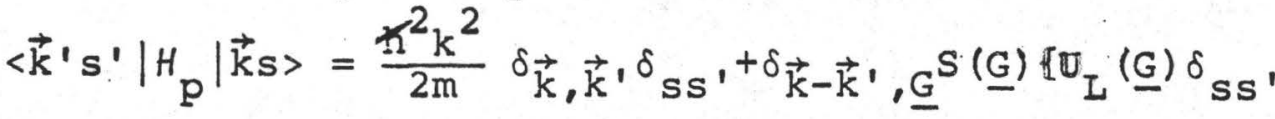

$$
\begin{aligned}
& \left.+v_{N}\left(\vec{k}, \vec{k}^{\prime}\right) \delta_{s s^{\prime}}+W_{s o}\left(\vec{k} s, \vec{k}^{\prime} s^{\prime}\right)\right]
\end{aligned}
$$


where $S(\underline{G})$ is a structure factor, s's are spin indices, $U_{L^{\prime}}, V_{N^{\prime}}$ and $W_{\text {so }}$ correspond to a local potential, a mon local potential and the spin orbit interaction respectively. Jine non local potentia]. is a sum of projection operators over the outer $s, p$ and d core states.

$$
\mathrm{V}_{\mathrm{N}}=\sum_{t} \mathrm{v}(\mathrm{t})|\mathrm{t}\rangle\langle t|
$$

In this expression $v(t)$ are disposable constants. In the case of zinc and cadmium it was found that $\mathrm{v}(\mathrm{s})$ could be set equal to zero, effectively grouping the s non local term with the local pseudopotential. For zinc $v(p)$ was also set at zero, but for cadmium $\mathrm{v}(\mathrm{p})$ came to the relativel $\Psi^{\prime}$ small value of 0.38 Ryabergs. In both cases the contribution from the outer d level was large, especially in zinc where it was 3.1.5 Ryabergs, in cadmium it was smaller being 1.78 Rydbergs.

In these substances it appears that the d-like contribution of the non-local pseudopotential is especially important, particularly for those portions of the Fermi surface where the wave functions have an appreciable d like character. If the outermost a levels of the core have energies only a little below the conduction band it is possible that the perturbation of the crystal field may hybrioize a significant amount of the core $\mathrm{d}$ wave function into the conduction band wave functions producing a strong repulsike d like perturbation.

In any particular case it is not immediately clear what approximation will produce a good psendopotential. To 
some extent it can be legitimate to proceed in a pragmatic experimental fashion.

\section{Spin-Orbit Coupling}

A method for including spin-orbit coupling in the pseudopotential formalism has been given by Weisz (1966). The theory is developed in a way analogous to that already described.

Beginning with equation (2.4) which was given for the no spin situation

$$
H(1-P)\left|\phi_{k}>=E_{k}(1-P)\right| \phi_{k}>\text {. }
$$

This can be transformed to the more symmetrical form.

$$
(1-P) H(1-P)\left|\phi_{k}>=E_{k}(1-P)^{2}\right| \phi_{k}>\text {. }
$$

However $\mathrm{P}$ is a projection operator, and so is (1-P). This means that $\mathrm{P}^{2}=\mathrm{P}$ and also $(1-\mathrm{P})^{2}=(1-\mathrm{P})$. Equateion (2.35) now becomes

$$
(I-P) H(I-P)\left|\phi_{k}>=E_{k}(I-P)\right| \dot{\phi}_{k}>
$$

The theory leading up to equation (2.36) can be extended to the more general case in which the total. Hamiltonian can be written as a sum of two parts.

$$
H_{\mathrm{p}}^{\mathrm{S}-\mathrm{O}}=H_{\mathrm{O}} \mathrm{I}(\mathrm{S})+H^{\mathrm{S}-\mathrm{O}} \text {, in which } H^{\mathrm{S}-\mathrm{O}}
$$

is the term that represents spin-orbit coupling. It mixes the spin up and spin down components of the wave function which is now represented by a two component spinor. I ${ }^{(s)}$ is the identity operator in spin space. 
The projection operator in equation (2.36) has so far been implied to mean the operator projecting on to the core states of the total Hamiltonian $H$. But if it is assumed that the actual core states are linear combinations in spin space of the no spin core functions, then the projection operator for the space spanned by the core states is

$$
P=I \stackrel{(s)}{\sum_{\alpha}|\alpha><\alpha|}
$$

where $|\alpha\rangle$ is now understood to mean the two component spinor.

$$
|\alpha\rangle \equiv\left(\psi_{\alpha}(\underline{r}) \uparrow, \psi_{\alpha}(r) \psi\right) .
$$

Substituting from equation (2.37) into equation (2.36), it being understood that the equations are now being applied to spin space.

$$
\left[(1-P) H_{0}(1-P)+(1-P) H^{S-O}(I-P)\left|\phi_{k}>=E_{k}(I-P)\right| \phi_{k}>\right.
$$

which can be rearranged to the form

$$
\left[\left(H_{0}+\left(E_{k}-H_{0}\right) P\right)+(I-P) H^{S-0}(I-P)\right]\left|H_{k}>=E_{k}\right| \phi_{k}>.
$$

The first term of the left hand side of the equation is now in the form of the pseudohamiltonian of equation (2.5) so that the spin-orbit pseudohamiltonian is equal to,

$$
\left.H_{\mathrm{p}}^{\mathrm{s}-0}=H_{\mathrm{p}}^{\mathrm{O}}+(1-\mathrm{P}) H^{\mathrm{s}-0}(1-\mathrm{P})\right)
$$

where $H_{p}{ }^{\circ}$ is the original no spin pseudohmiltonian $H_{p}$ multiplied by $I^{(s)}$.

If the basis set of plane waves with spin up and spin down components is selected, a typical matrix element will be 
given by

$H_{p_{\left(k^{\prime} s^{\prime}, k s\right)}^{s-0}}^{s}=\left\langle k^{\prime}\left|H_{p}\right| k>\delta_{s^{\prime} s}+\left\langle k^{\prime} s^{\prime}\left|(I-P) H^{s-0}(I-P)\right| k s\right\rangle\right.$

The last term which explicitly represents spin-orbit coupling can be written out in full.

$$
\begin{aligned}
& \left\langle k^{\prime} S^{\prime}\left|(I-P) H^{S-O}(I-P)\right| k S\right\rangle=\left\langle k^{\prime} S^{\prime}\left|H^{S-O}\right| k S\right\rangle \\
& -\sum\left\langle\mathrm{k}^{\prime} \mathrm{s}^{\prime} \mid \alpha\right\rangle\left\langle\alpha\left|H^{\mathrm{s}-\mathrm{O}}\right| \mathrm{ks}\right\rangle \\
& \alpha \\
& -\Sigma\left\langle k^{\prime} s^{\prime}\left|H^{S-O}\right| \alpha\right\rangle\langle\alpha \mid k s\rangle \\
& \alpha \\
& +\sum_{\alpha \alpha^{\prime}}\left\langle k^{\prime} S^{\prime} \mid \alpha\right\rangle\left\langle\alpha\left|H^{S-O}\right| \alpha^{\prime}>\left\langle\alpha^{\prime} \mid k s\right\rangle \ldots\right.
\end{aligned}
$$

The spin-orbit Hamiltonian is given by an approximation derived from the Dirac relativistic theory.

$$
H^{S-O}=\frac{\hbar^{2}}{4 \mathrm{~m}^{2} \mathrm{C}^{2}}(\nabla \mathrm{V} \times \underline{\underline{p}} \cdot \underline{\sigma})
$$

in which $\nabla V$ is the gradient of the real potential and $\underline{\sigma}$ is the Pauli spin operator.

Earlier OPW calculations have shown that for states that possess an \& symmetry already included in the core states the double summation core-core term of equation (2.40) makes the largest contribution. In magnesium the core-core and coreplane wave terms are reposted to account for 99\% or more of the contributions.

The biggest contribution to the integrals comes from the singularity in $\nabla V$ near the nuclei. In this region the overlap of core orbitals and potentials is negligible, allowing 
$H^{\mathrm{S}-\mathrm{O}}$ to be expressed as a sum of atomic Hamiltonians.

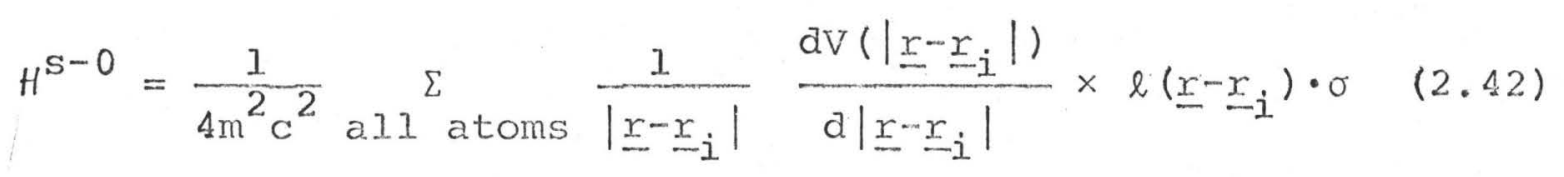

Using this approximation Weisz has obtained the final formula $H_{k^{\prime} s^{\prime}, k s}^{s-0}=s\left(k-k^{\prime}\right)\left[\frac{\not^{2}}{4 m c^{2}} U\left(\left|k^{\prime}-k\right|\right)-\lambda p^{-\lambda}\left(k^{\prime} \cdot k\right)\right] \times i k{ }^{\prime} \times k \cdot \sigma_{s^{\prime}} s$

The term involving the crystal potential U comes from the matrix elements of the spin-orbit Hamiltonian between two plane waves. It is believed to contribute $1 \%$ or less to the total spin-orbit matrix element. For this reason it is often neglected. The positive constants $\lambda_{p}$ and $\lambda_{d}$ account for the contributions of the core $\mathrm{p}$ and $\mathrm{d}$ states. If the state under consideration had a considerable f-like symmetry component and there was an $f$ like core state then another term would have to be included in the midale bracket of equation (43) to put it into the form

$$
\frac{\varkappa^{2}}{4 m c^{2}} U\left(\left|\underline{k}{ }^{\prime}-\underline{k}\right|\right)-\lambda_{p}-\lambda_{d}\left(\underline{\left.k^{\prime} \cdot \underline{k}\right)}-\lambda_{f}(\underline{k} \cdot \cdot \underline{k})^{2}\right. \text {. }
$$

Which spin-orbit parameters should be included depends upon the symmetry character of the state bejng considered. If the conduction band wave function were entirely s like it would be possible to set $\lambda_{p}, \lambda_{d}, \lambda_{f}$ all equal to zero. As the remaining term is very small in any case, s like states will. be very little affected by spin-orbit coupling. If the wave function were mainly $p$ like in character then to a good approxi- 
mation it would be sufficient to put $\lambda_{p}$ as the only non zero parameter and so on.

8. Explicit Calculation of the Spin-orbit Coupling Matrix Elements

The matrix element for spin-orbit coupling is

$H_{k^{\prime} s^{\prime}, k s^{s}}^{s-o}=S\left(\underline{k}-k^{\prime}\right)\left[\frac{\hbar^{2}}{4 m c^{2}} U\left(\left|\underline{k}-\underline{k}^{\prime}\right|\right)-\lambda_{p^{\prime}}-\lambda d\left(\underline{k}^{\prime} \cdot \underline{k}\right)\right] \times i \underline{k} \underline{\prime}^{\prime} \times \underline{k} \cdot \sigma_{s^{\prime}} s$

It is implied in this notation that the indices $s$ and $s$ ' are equal to 1 or 2 . The spin up state $\uparrow$ being represented by 1 , and the spin down state $\downarrow$ by 2 .

Since $\underline{\sigma}, \underline{k}$ and $\underline{k}$ can all be written in the form,

$$
\underline{\sigma}=\sigma_{x \underline{i}}+\sigma_{y} \underline{j}+\sigma_{z} \underline{k}
$$

(i, $\dot{j}, \underline{k}$ being a right-handed triad of unit cartesian vectors) and $\left(\underline{k}^{\prime} \times \underline{k}\right)=\left(k_{y}{ }^{\prime} k_{z}-k_{y} k_{z}^{\prime}, k_{z}{ }^{\prime} k_{x}-k_{z} k_{x}^{\prime}, k_{x}{ }^{\prime} k_{y}-k_{x} k_{y}^{\prime}\right)$. Therefore,

$$
\begin{gathered}
\left(\underline{k}^{\prime} \times \underline{k}\right) \cdot \underline{\sigma}_{s^{\prime} s}=\left(k_{y}{ }^{\prime} k_{z}-k_{y} k_{z}^{\prime}\right) \sigma_{x}^{s^{\prime} s}+\left(k_{z} k_{x}-k_{z} k_{x}^{\prime}\right) \sigma_{y}^{s^{\prime} s} \\
+\left(k_{x} k_{y}-k_{x^{k}}{ }^{\prime}\right) \sigma_{z}^{s^{\prime} s}
\end{gathered}
$$

In matrix notation $\left(\begin{array}{l}1 \\ 0\end{array}\right)$ represents the spin up state $|\uparrow\rangle$ and $\left(\begin{array}{l}0 \\ 1\end{array}\right)$ the spin down state $|\psi\rangle$. The Pauli spin matrices are given by

$$
\sigma_{x}=\left(\begin{array}{ll}
0 & 1 \\
1 & 0
\end{array}\right), \sigma_{y}=\left(\begin{array}{cc}
0 & -i \\
i & 0
\end{array}\right), \sigma_{z}=\left(\begin{array}{rr}
1 & 0 \\
0 & -i
\end{array}\right) \text {. }
$$

It follows immediately that

$$
\sigma_{x}|\uparrow\rangle=|\uparrow\rangle, \sigma_{x}|\uparrow\rangle=|\uparrow\rangle
$$




$$
\begin{aligned}
& \sigma_{y}|\uparrow\rangle=i|\downarrow\rangle, \sigma_{y}|\downarrow\rangle=-i|\uparrow\rangle \\
& \sigma_{z}|\uparrow\rangle=|\uparrow\rangle, \sigma_{z}|\downarrow\rangle=-|\downarrow\rangle
\end{aligned}
$$

This means that

$$
\begin{aligned}
& \left\langle\uparrow\left|\sigma_{x}\right| \uparrow\right\rangle=\sigma_{x}^{1,1}=0 ;\left\langle\uparrow\left|\sigma_{x}\right| \downarrow\right\rangle=\sigma_{x}^{1,2}=1 . \\
& \left\langle\downarrow\left|\sigma_{x}\right| \uparrow\right\rangle=\sigma_{x}^{2,1}=1 ;\left\langle\downarrow\left|\sigma_{x}\right| \downarrow\right\rangle=\sigma_{x}^{2,2}=0 . \\
& \left\langle\uparrow\left|\sigma_{y}\right| \uparrow\right\rangle=\sigma_{y}^{1,1}=0 ;\left\langle\uparrow\left|\sigma_{y}\right| \downarrow\right\rangle=\sigma_{y}^{1,2}=-i . \\
& \left\langle\downarrow\left|\sigma_{y}\right| \uparrow\right\rangle=\sigma_{y}^{2,1}=i ;\left\langle\downarrow\left|\sigma_{y}\right| \downarrow\right\rangle=\sigma_{y}^{2,2}=0 . \\
& \left\langle\uparrow\left|\sigma_{z}\right| \uparrow\right\rangle=\sigma_{z}^{1,1}=1 ;\left\langle\uparrow\left|\sigma_{z}\right| \downarrow\right\rangle \quad \sigma_{z}^{1,2}=0 . \\
& \left\langle\downarrow\left|\sigma_{z}\right| \uparrow\right\rangle=\sigma_{z}^{2,1}=0 ;\left\langle\downarrow\left|\sigma_{z}\right| \downarrow\right\rangle \quad \sigma_{z}^{2,2}=-1 .
\end{aligned}
$$

From these formulae it follows that

$$
\begin{aligned}
& \left\langle\underline{k} l\left|H^{s-0}\right| \underline{k^{\prime}} l>=i s\left(\underline{k}{ }^{\prime}-\underline{k}\right) F\left(\underline{k}, k^{\prime}\right)\left(k_{x}{ }^{\prime} k_{y}-k_{y}{ }^{\prime} k_{x}\right)\right. \\
& \left.\langle\underline{k}]\left|H^{s-0}\right| \underline{k}^{\prime} 2\right\rangle=i s\left(\underline{k}^{\prime}-\underline{k}\right) F\left(\underline{k}, \underline{k}^{\prime}\right)\left[\left(k_{y}{ }^{\prime} k_{z}-k_{z}{ }^{\prime} k_{y}\right)\right. \\
& \left.-i\left(k_{z}{ }^{\prime} k_{x}-k_{x}{ }^{\prime} k_{z}\right)\right] \\
& \left\langle\underline{\mathrm{k}} 2\left|H^{\mathrm{s}-0}\right| \underline{\mathrm{k}}^{\prime} l\right\rangle=\operatorname{is}\left(\underline{\mathrm{k}} \underline{ }^{\prime}-\underline{\mathrm{k}}\right) \mathrm{F}\left(\underline{\mathrm{k}}, \underline{\mathrm{k}}^{\prime}\right)\left[\left(\mathrm{k}_{\mathrm{y}}{ }^{\prime} \mathrm{k}_{\mathrm{z}}-\mathrm{k}_{\mathrm{z}}{ }^{\prime} \mathrm{k}_{\mathrm{y}}\right)\right. \\
& \left.+i\left(k_{z}{ }^{\prime} k_{x}-k_{x}{ }^{\prime} k_{z}\right)\right] \\
& \left\langle\underline{k} 2\left|H^{s-0}\right| \underline{k^{\prime}} 2\right\rangle-i S\left(\underline{k}{ }^{\prime}-\underline{k}\right) F\left(\underline{k}, \underline{k}^{\prime}\right)\left(k_{x}{ }^{\prime} k_{y}-k_{y}{ }^{\prime} k_{x}\right)
\end{aligned}
$$

where

$$
F\left(\underline{k}, \underline{k}^{\prime}\right)=-\frac{\hbar^{2}}{4 m c^{2}} U\left(\left|\underline{k}-\underline{k}^{\prime}\right|\right)+\lambda_{p}+\lambda_{d}\left(\underline{k}^{\prime} \cdot \underline{k}\right) .
$$

The general spin-orbit coupling matrix element for the full pseudohamiltonian is given by equation (2.39),

$$
\left\langle\underline{k} s\left|H_{p}^{s-0}\right| \underline{k}^{\prime} s^{\prime}\right\rangle=\left\langle\underline{k}\left|H_{p}\right| \underline{k}^{\prime}\right\rangle \delta s_{s}+\left\langle\underline{k} s \| H^{s-0} \mid k^{\prime} s^{\prime}\right\rangle .
$$

The equations (2.47) provide the expressions for the spin dependent term on the right of (2.39). 
9. The Repulsive Potential of the Inner-core States

It is implicitly assumed in this thesis that the effect of the orthogonalization to the inner core states can be represented by an effective repulsive potential that can be lumped together with the real potential. The following arguments are presented to justify this assumption and also to emphasize the physical importance of such effects as screening.

The OPW-type of pseudopotential given in equation (2.7) has a pseudohamiltonian of the form,

$$
H_{p}=T+V(\underline{r})+\sum_{\alpha}\left(E_{k}-E_{\alpha}\right)|\alpha><\alpha|
$$

The matrix elements that arise when this pseudopotential is being used are given by equation (2.23),

$$
\begin{aligned}
& \left\langle\underline{\mathrm{k}}+\underline{G}_{1}\left|H_{\mathrm{p}}\right| \underline{\mathrm{k}+\underline{G}_{2}}\right\rangle=\left|\underline{\mathrm{k}}+\underline{G}_{1}\right|^{2} \delta_{\underline{G}_{I}, \underline{G}_{2}}+\frac{\mathrm{l}}{\Omega} U\left(\underline{G}_{I}-\underline{G}_{2}\right) \\
& +\frac{4 \pi}{\Omega} \sum_{n, l}\left(E_{k}-E_{n \ell}(2 \ell+1) P_{\ell}\left(\cos \left(k+G_{1}, k+G_{2}\right)\right)\right. \\
& \times \int j_{\ell}\left(\left|\underline{k+G_{I}}\right| r\right) r P_{n \ell}(r) d r \times \int j_{\ell}\left(\left|\underline{k}+\underline{G}_{2}\right| r\right) r P_{n \ell}(r) d r .
\end{aligned}
$$

It will be converient to adopt the notation that

$$
\left\langle\psi_{n \ell} \mid k\right\rangle=\int j_{\ell}(k x) r P_{n \ell}(x) d x .
$$

In terms of this notation equation (2.23) can be rewritten in the form 


$$
\begin{aligned}
& \left\langle\underline{\mathrm{k}+\underline{G}_{1}}\left|\mathrm{H}_{\mathrm{p}}\right| \underline{\mathrm{k}}+\underline{G}_{2}\right\rangle=\left|\underline{\mathrm{k}}+\underline{G}_{1}\right|^{2} \delta_{\underline{G}_{1}, \underline{G}_{2}}+\frac{1}{\Omega} \mathrm{U}\left(\underline{G}_{1}-\underline{G}_{2}\right) \\
& +\frac{4 \pi}{\Omega} \sum_{n, \ell}\left(E_{k}-E_{n \ell}\right)(2 \ell+I) P_{\ell}\left(\cos \left(\underline{k}+\underline{G}_{1}, k+G_{2}\right)\right) \\
& \times<\underline{\mathrm{k}}+\underline{\mathrm{G}}_{\mathrm{I}} \mid \psi_{\mathrm{n} \ell}>\left\langle\psi_{\mathrm{n} \ell}\right| \underline{\mathrm{k}}+\underline{\mathrm{G}}_{2}>
\end{aligned}
$$

Figure (II, I) shows the variation of the form factors $\langle 5 \mathrm{~s} \mid \mathrm{k}\rangle,\langle 5 \mathrm{p} \mid \mathrm{k}\rangle,\langle 5 \mathrm{~d} \mid \mathrm{k}\rangle,\langle 4 \mathrm{~s} \mid \mathrm{k}\rangle,\langle 4 \mathrm{p} \mid \mathrm{k}\rangle$, and $\langle 4 \mathrm{~d} \mid \mathrm{k}\rangle$ as a function of wave number $|\underline{k}|$, for the atomic wave functions of mercury. The points $I, X$, and $T$ on the axis of this graph show the magnitudes of the reciprocal lattice vectors which are bisected by the $\mathrm{L}, \mathrm{X}$, and $\mathrm{T}$ faces of the Brillouin zone of mercury which is illustrated in Figure (III.I).

Most of the important matrix elements involve values of $|\underline{k}|$ which are less than approximately 2.5 (reciprocal atomic units). In any particular matrix element there are two of these form factors which are multiplied together in the fashion $\left\langle\underline{\mathrm{k}}+\underline{G}_{1}\right| \psi_{\mathrm{n} \ell}>\left\langle\psi_{\mathrm{n} \ell} \mid \underline{\mathrm{k}}+\mathrm{G}_{2}\right\rangle$. It can be seen that the form factors of the $5 \mathrm{~s}, 5 \mathrm{p}$, and $5 \mathrm{~d}$ states vary a great deal over the important range of $\underline{k}$. The $4 \mathrm{~s}$ form factor varies much more slowly, while the $3 \mathrm{~s}, 2 \mathrm{~s}$, and $1 \mathrm{~s}$ form factors are almost constant over this range of $\underline{k}$.

Another important observation is that the $4 \mathrm{p}$ and $4 \mathrm{~d}$ form factors are relatively small when $\mathrm{k}$ is small, and when the product of two of them is taken the result is even smaller. The form factors for the states $3 p, 3 d, 2 p$, and $4 f$ are 


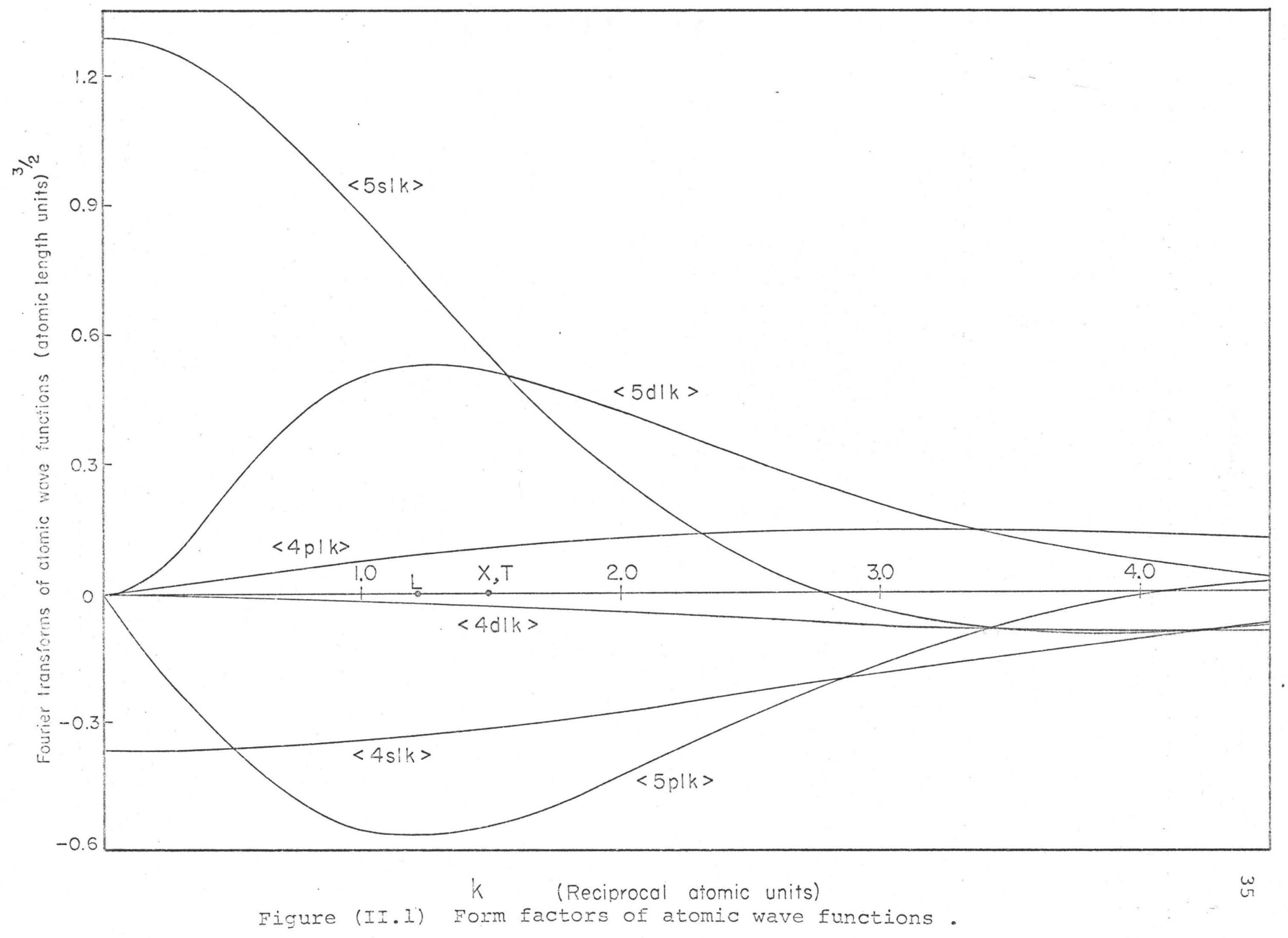


relatively negligible over the range of interest.

In this thesis all calculations involving the use of atomic wave functions make use of the tabulated radial wave functions of Herman and skiliman.

Table (II.I) shows the variation of the Fourier transform form factors for the different atomic wave functions. The energies listed are the atomic energy levels derived by Herman and Skillman.

The effective repulsive potential of an inner-core state is given by the term

$$
\begin{aligned}
& \frac{4 \pi}{\Omega}\left(E_{k}-E_{n \ell}\right)(2 \ell+1) P_{\ell}\left(\cos \left(\underline{k}+G_{I}, \underline{k}+\underline{G}_{2}\right)\right) \\
& \left.\times<\underline{k}_{+}+\underline{G}_{2}\left|\psi_{n \ell}><\psi_{n \ell}\right| \underline{k}_{+} \underline{G}_{2}\right\rangle .
\end{aligned}
$$

This effective repulsive potential depends upon the angle between the two vectors $\left(\underline{k}+\underline{G}_{1}\right)$, and $\left(\underline{k}+\underline{G}_{2}\right)$, through the Legendre polynomials. However for s-states, the Legendre polynomial is always equal to unity and there is no angular dependence in the effective repulsive potential. States for which $\ell=1,2,3, \ldots$, do have an angulax dependence, but these states have very small form factors when $k$ is small This means that the effective repulsive potential mostly comes from the s-states in the core.

The atomic form factors of the inner-core s-states are almost constant over the range of $\underline{k}$, which is important, this means that they can be approximated by their value at $\underline{k}=0$. 
TABLE (II. I)

A comparison of the Fourier transforms of the radial part of the Herman-Skillman atomic wave functions

\begin{tabular}{|c|c|c|c|c|c|c|c|}
\hline \multirow{2}{*}{$\begin{array}{l}\text { Atomic } \\
\text { Level }\end{array}$} & \multirow{2}{*}{$\begin{array}{l}\text { Energy } \\
\text { (Rydbergs) }\end{array}$} & \multicolumn{2}{|c|}{ Value } & (reciprocal & ato & \multicolumn{2}{|l|}{ units) } \\
\hline & & 0 & 1 & 2 & 3 & 4 & 5 \\
\hline $5 d$ & -1.2703 & 0 & 0.5018 & 0.4239 & 0.2067 & 0.0737 & 0.0107 \\
\hline $5 p$ & -5.0123 & 0 & -0.5512 & -0.4298 & -0.1619 & -0.0120 & 0.042 \\
\hline $5 s$ & -7.3714 & 1.293 & 0.8856 & 0.2619 & -0.0438 & -0.1037 & -0.0754 \\
\hline $4 f$ & -9.386 & 0 & 0.0049 & 0.0272 & 0.0513 & 0.0659 & 0.0697 \\
\hline $4 d$ & -27.430 & 0 & -0.0141 & -0.0465 & -0.0771 & -0.0935 & -0.0944 \\
\hline $4 p$ & -40.749 & 0 & 0.0778 & 0.1309 & 0.1489 & 0.1373 & 0.1091 \\
\hline $4 \mathrm{~s}$ & -47.934 & -0.3715 & -0.3455 & -0.2778 & -0.1913 & -0.1090 & $-0.04 \Delta 7$ \\
\hline $3 d$ & -173.33 & 0 & 0.0006 & 0.0023 & 0.0048 & 0.0079 & 0.0112 \\
\hline $3 p$ & -204.12 & 0 & -0.0110 & -0.021 .2 & -0.0300 & -0.0368 & -0.0415 \\
\hline $3 s$ & -220.49 & 0.1210 & 0.1191 & 0.1136 & 0.1050 & 0.0941 & 0.0816 \\
\hline $2 p$ & -896.92 & 0 & 0.00 .115 & 0.0023 & 0.0034 & 0.0044 & 0.0054 \\
\hline $2 s$ & -930.98 & -0.0354 & $1-0.0353$ & -0.0350 & -0.0345 & -0.0338 & -0.033 \\
\hline Is & -5535.7 & 0.00568 & 0.00568 & 0.00568 & 0.00567 & 0.00566 & 0.00564 \\
\hline
\end{tabular}


Using this approximation the effective repulsive potential of an inner core s-state is given by, Effective repulsive potential $=\frac{4 \pi}{\Omega} \cdot\left(E_{k}-E_{S}\right)\left\langle k \mid \psi_{S}\right\rangle\left\langle\psi_{S} \mid k\right\rangle$

$$
\text { evaluated at } k=0 .(2.51)
$$

These effective repulsive potentials have almost the same value in every matrix element and they have the effect of adding a constant positive term on to the local pseudopotential $\frac{1}{\Omega} U\left(\underline{G}_{1}-\underline{G}_{2}\right)$.

If $\mathrm{E}_{\mathrm{k}}$ is taken as the energy of the $6 \mathrm{~s}$ atomic energy level, then $\left(\mathrm{E}_{\mathrm{K}}-\mathrm{E}_{\mathrm{S}}\right)$ can be calculated for each inner-core s state and an order of magnitude estimate obtained for the strength of the repulsive forces. The actual energy levels in the solid will not be exactly the same as for the free atom, however the inner-core levels should not be strongly perturbed and the use of atomic energy levels should be a sufficiently good approximation. This will not necessarily be true for the $5 s, 5 p$, and $5 d$ outer core states which may be quite strongly affected by the crystal field.

The effective repulsive potentials of the inner-core s-states have been calculated and are given in Table (II.2). These repulsive potentials tend to cancel out the attractive potentials of the ionic potential wells, however screening also plays a very important role in determining the strength of the pseudopotential. 
TABLE (II.2)

The effective repulsive potential of inner-core s-states. (Energies in Ryabergs). $\mathrm{E}_{6 \mathrm{~S}}^{\dagger}=-0.5653$

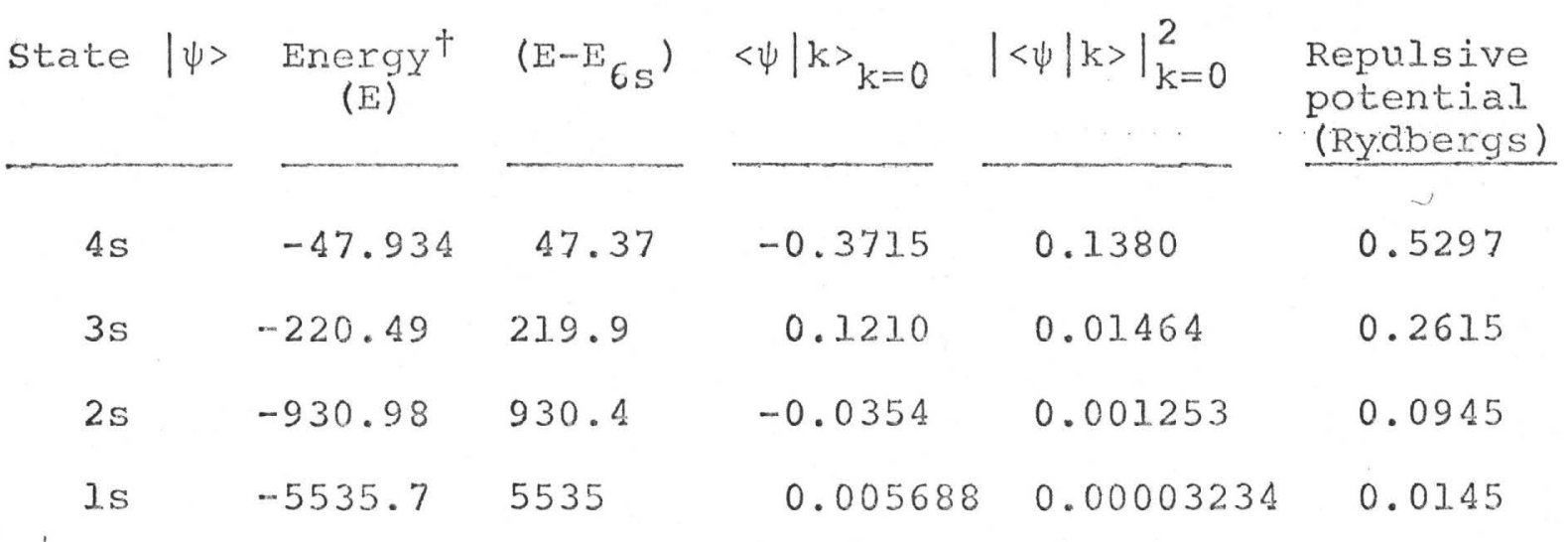

Total repulsive potential from $4 \mathrm{~s}, 3 \mathrm{~s}, 2 \mathrm{~s}$, and $1 \mathrm{~s}$ inner core states $=0.9002$ Rydbergs.

Repulsive potential of $5 \mathrm{~s}$ state at $k=0$

\begin{tabular}{|c|c|c|c|c|c|}
\hline state & $\begin{array}{l}\text { Energy } \\
(\mathrm{E})\end{array}$ & $\cdot\left(E-E_{6 s}\right.$ & $\langle\psi \mid k\rangle_{k=0}$ & $|\langle\psi \mid k\rangle|_{k=0}^{2}$ & $\begin{array}{l}\text { Repulsive } \\
\text { potential }\end{array}$ \\
\hline $5 s$ & -7.3714 & 6.806 & 1.293 & 1.672 & 0.9217 \\
\hline
\end{tabular}

Total repulsive potential at $k=0,=1.822$ Rudbergs

+ , Atomic energy levels have been taken from Herman and skiliman. 
The coulomb potential (relative to an electron) of a point charge $z e$ is given by $-\frac{z e^{2}}{r}$, which means that $V(r)=-\frac{2 z}{r}$, if energies are expressed in Rydbergs and distances in atomic units.

Herman and skillman make use of a normalized quantity $\mathrm{U}(r)$ to express the atomic potential,

$$
U(x)=-\frac{r V(x)}{2 Z}, \begin{aligned}
& (z \text { is the atomic number of the } \\
& \text { element }) .
\end{aligned}
$$

$U(r)$ is equal to unity near the nucleus where the full nuclear charge determines the potential. As $r$ increases $U(r)$ gradually falls because of the screening of the nuclear charge by the electrons of the atomic core.

In the interior of the ion the Herman-Skillman atomic potential is given by,

$$
V_{0}(r)=-\frac{2 Z}{r}-\frac{2}{r} \int_{0}^{r} \sigma(t) \frac{d t}{t}-6\left[-\frac{3}{8 \pi} \rho(x)\right]^{(1 / 3)} \quad \text { (2.52) }
$$

where $\rho(x)=\left(4 \pi r^{2}\right)^{-1} \sigma(x)$ is the spherically averaged total electronic charge density (both spins). The quantity $\sigma(r)$ is given by

$$
\sigma(r)=-\sum_{n, \lambda} \omega_{n \lambda}\left[p_{n \lambda}(r)\right]^{2}
$$

$\omega_{n \lambda}$ is the occupation number for the orbital $(n, \lambda)$ when both spins are included. In the case of closed shells $\omega_{n \lambda}=2(2 \lambda+1)$. The last term in equation (2.52) is the slater free electron exchange potential. It is a correction term added to the atomic potential as an approximation to include the effect of 
exchange. If the total number of electrons on the ion is equal to $\mathrm{N}$, the ionic charge will be equal to $(\mathrm{Z}-\mathrm{N})$.

The atomic potentials of Herman and skillman were assumed to be given by the approximation,

$$
\begin{aligned}
& V(r)=V_{0}(r), r<r_{0} \\
& V(r)=-2(z-N+I) / r, r \geq r_{0} .
\end{aligned}
$$

The radius $r_{0}$ is defined by the equality

$$
V_{0}\left(r_{0}\right)=-2(Z-N+1) / r_{0} \text {. }
$$

The value of $r_{0}$ is a measure of the ionic radius, for mercury $r_{0}=3.398$ atomic units, or $r_{0}=1.798 \AA$.

The Fourier transform of the atomic potential is given by

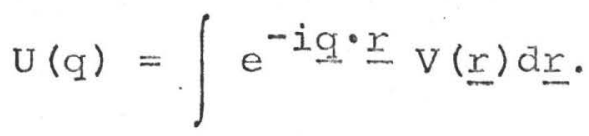

This can be transformed to the expression

$$
U(q)=-\frac{8 \pi z}{q} \int_{0}^{r_{0}} U(r) \sin (q r) d r-\frac{8 \pi z}{q} \int_{r_{0}}^{\infty} U_{c} \sin (q r) d r
$$

In this last equation $U_{C}$ is the constant value which $U(r)$ assumes when $r \geq r_{0}$

The quantity of interest which enters in equation (2.49)

is $V(q)$, where $V(q)=U(q) / \Omega$. $\Omega$ is the volume of a unit cell which in the case of mercury is equal to 22.986 cubic angstroms.

The function $V(q)$ is shown in Figure (II.2). In this graph the value of $V(q)$ is seen to tend towards $-\infty$ as $q \rightarrow 0$. In fact as $q \rightarrow 0$, 


$$
V(q) \rightarrow-\frac{8 \pi z}{\Omega} \int_{0}^{r_{O}} U(r) x d r-\frac{8 \pi z}{\Omega} \frac{U_{C}}{q^{2}}
$$

The divergent behaviour of the function $V(q)$ arises because of the second term in equation $(2.54)$. It is related to the fact that the ionic coulomb potential falls off relatively slowly as $\frac{l}{r}$ and is a long range potential.

In fact the screening by the conduction electrons limits the effective range of the ionic potentials to the immediate neighbourhood of the unit cell in which a particular ion is located. The screening can be approximately treated by dividing $V(q)$ by $\varepsilon(q)$, where $\varepsilon(q)$ is the Hartree dielectric screening function given in equation (2.32).

$$
\varepsilon(q)=1+\frac{1}{2 \pi k_{F} n^{2}}\left(\frac{1-n^{2}}{2 \eta} \log _{e}\left|\frac{1+n}{1-n}\right|+1\right)
$$

where $n=\frac{q}{2 k_{F}}, k_{F}=$ the radius of the free electron sphere. This function is also shown in Figure (II.2). The important property of $\varepsilon(q)$ is that it also tends towards infinity as $\frac{1}{q^{2}}$, as $q \rightarrow 0$.

$$
\varepsilon(q)+\left(\frac{4 k F}{\pi}\right) \frac{l}{q^{2}}, q+0 .
$$

If $V(q)$ of Figure (II.2) is divided by $\varepsilon(q)$ the resulting atomic form factor will be very unphysical. It seems reasonable to suppose that the screening processes axe unim-. portant inside the ionic cores.

$\mathrm{V}(\mathrm{q})$ can be written as the sum of two parts 


$$
V(q)=V_{\text {core }}(q)+V_{\text {extra-core }}(q),
$$

where

$$
v_{\text {core }}(q)=-\frac{8 \pi z}{q \Omega} \int_{0}^{r} u(r) \sin (q r) d r,
$$

and

$$
v_{\text {extra-core }}(q)=-\frac{8 \pi z}{q \Omega} \int_{r_{0}}^{\infty} u(r) \sin (q r) d r .
$$

The approximation was adopted that the effective atomic form factor will be given by the approximation

$$
W_{S}(q)=V_{\text {core }}(q)+\frac{V_{\text {extra-core }}(q)}{\varepsilon(q)}
$$

The screened atomic form factor $W_{S}(q)$ is shown in Figure (II.3). The parallel curve $\mathrm{W}_{\mathrm{S}}{ }^{*}(q)$ is the value of the atomic form factor when the repulsive potentials of the inner core $(4 s, 3 s, 2 s, 1 s)$ s-states are taken into account.

The preceding arguments are approximate they are presented as an attempt to estimate the order of magnitude of the various terms involved in an OPW type pseudopotential. 


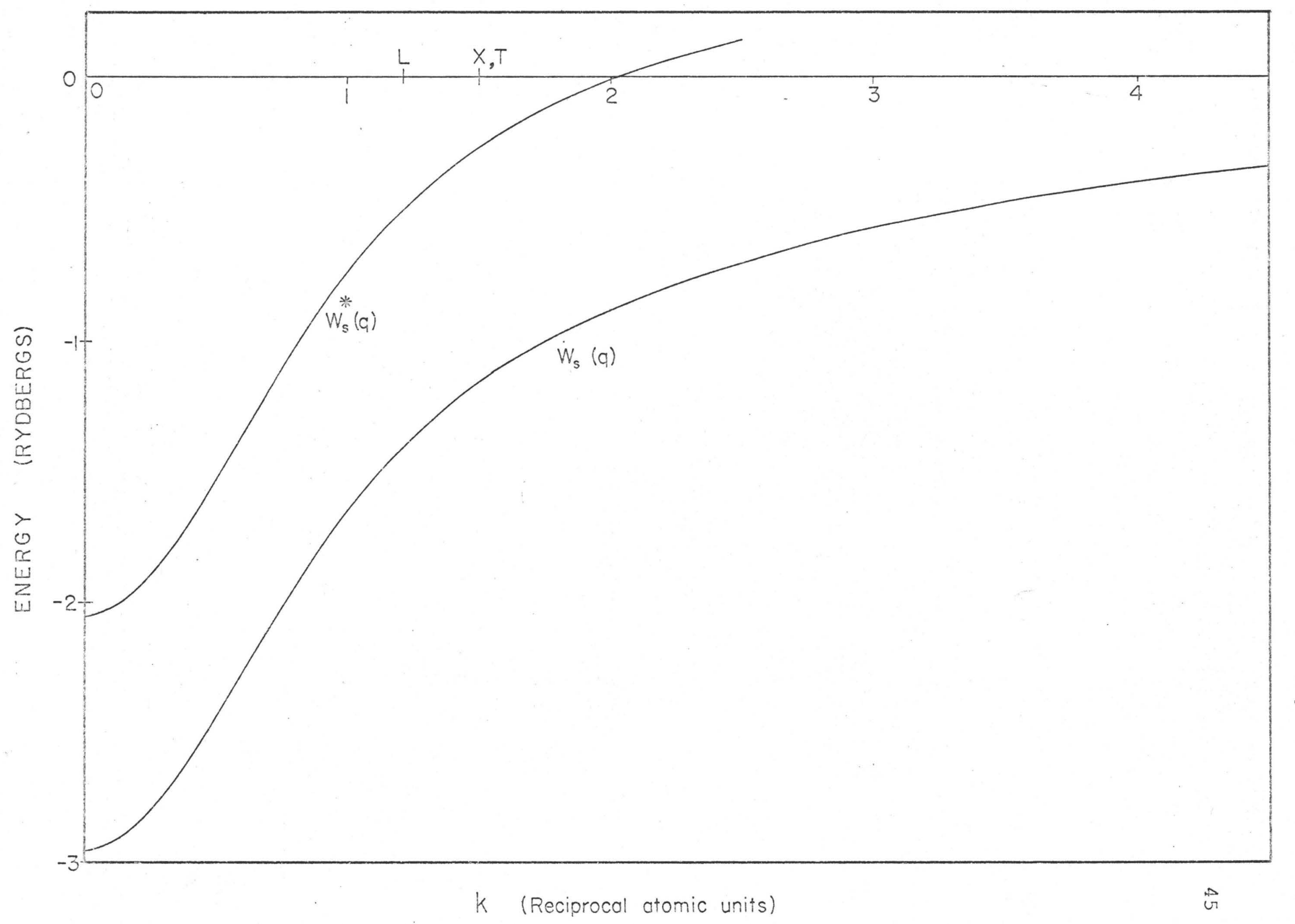

Figure (II.3) Form factors of screened atomic potentials. 
CHAPTER ITI

GENERAL, CHARACTERISTICS OF THE ENERGY BANDS OF MERCURY

1. Introduction

The simplest approximation that it is possible to make when computing the conduction electron energy bands in a metal is to assume that the electrons are completely free. In this approximation which contains virtually no physics at all, the electronic eigenstates are simply the plane wave states with a spin degeneracy.

$$
\psi_{\underline{k}}=\frac{1}{\sqrt{V}} e^{i \underline{k} \cdot \underline{x}} x_{i}
$$

$\mathrm{V}$ is the volume of the crystal.

$x$ is a spin function.

With energies given by,

$$
\mathrm{E}_{\mathrm{k}}=\underline{\mathrm{k}}^{2}
$$

using atomic units with energies in Rydbergs.

In the extended zone representation the occupied

states will be confined to a sphere of radius $k_{F}$ at $0^{\circ} \mathrm{K} ; k_{F}$ is called the Fermi radius. The free electron Fermi radius can be calculated by making use of periodic boundary conditions and using the pauli principle.

If the crystal has basis vectors $a_{1}, \underline{a}_{2}, a_{3}$ and it is assumed that it has the form of a parallelepiped with sides 
equal to $\mathrm{N}_{1} \underline{a}_{1}, \mathrm{~N}_{2} \underline{a}_{2}, \mathrm{~N}_{3} \underline{a}_{3}$ respectively. Then if periodic boundary conditions are imposed on the free electron wave function

Giving,

$$
\exp (\underline{i k} \cdot \underline{r})=\exp \left(\underline{i k} \cdot\left(\underline{r}+N_{1} \underline{a}_{1}+N_{2} \underline{a}_{2}+N_{3} \underline{a}_{3}\right)\right)
$$

So that,

$$
\begin{gathered}
1=\exp \left(\underline{i k} \cdot\left(N_{1} \underline{a}_{1}+N_{2} \underline{a}_{2}+N_{3} \underline{a}_{3}\right)\right) \\
\underline{k} \cdot\left(N_{1} \underline{a}_{1}+N_{2} \underline{a}_{2}+N_{3} \underline{a}_{3}\right)=2 \pi M, \\
\text { where } M \text { is any integer }
\end{gathered}
$$

If the reciprocal lattice vectors are represented by $\underline{b}_{1}, \underline{b}_{2}, \underline{b}_{3}$ which satisfy the relation

$$
\underline{a}_{i} \cdot \underline{b}_{j}=\delta_{i j}
$$

Then $k$ can be written as

$$
\underline{k}=\lambda_{1} \underline{b}_{1}+\lambda_{2} \underline{b}_{2}+\lambda_{3} \underline{b}_{3} .
$$

which on substituting into equation (3.1) gives

$$
\mathrm{N}_{1} \lambda_{1}+\mathrm{N}_{2} \lambda_{2}+\mathrm{N}_{3} \lambda_{3}=2 \pi \mathrm{M} .
$$

This means that the only permissible values of $\lambda_{1}, \lambda_{2}, \lambda_{3}$ are integral multiples of $2 \pi / N_{1}, 2 \pi / N_{2}, 2 \pi / N_{3}$ respectively.

In this way $k$ space is divided up into small cells

of volume $v_{c}$, where

$$
v_{C}=\frac{8 \pi^{3}}{\left(N_{1} N_{2} N_{3}\right)} b_{1} \cdot\left(\underline{b}_{2} \times \underline{b}_{3}\right)
$$

But

and

$$
\underline{b}_{1} \cdot\left(\underline{b}_{2} \times \underline{b}_{3}\right)=\frac{l}{\left(a_{1} \cdot\left(a_{2} \times a_{3}\right)\right.}
$$

$$
\begin{gathered}
\quad \mathrm{V}=\left(\mathrm{N}_{1} \mathrm{~N}_{2} \mathrm{~N}_{3}\right) \underline{a}_{1} \cdot\left(\underline{a}_{2} \times \underline{a}_{3}\right) . \\
\text { Giving } \\
v_{C}=\frac{8 \pi^{3}}{\mathrm{~V}} .
\end{gathered}
$$


The Pauli principle permits two electrons of opposite spin to each cell. This result for the density of allowed states in phase space is general and is not restricted to the special case of free electrons.

If each atom in the system contributes z valence electrons to the conduction band there will be $\mathrm{Nz}$ free electrons in the crystal. The free electron sphere must be large enough to accoinmodate this number of electrons.

$$
\frac{4 \pi}{3} \cdot k_{F}^{3} \cdot \frac{V}{8 \pi^{3}} \cdot 2=N z
$$

Or,

$$
\mathrm{k}_{\mathrm{F}}{ }^{3}=3 \pi^{2}\left(\frac{\mathrm{N}}{\mathrm{V}}\right) \mathrm{z}=3 \pi^{2} \frac{\mathrm{z}}{\mathrm{v}}
$$

$v=$ the volume of a unit cell of the crystal.

This gives the free electxon Fermi radius equal to,

$$
k_{F}=\left(3 \pi^{2} \frac{z}{v}\right)^{\left(\frac{l}{3}\right)}
$$

This spherical surface separating occupied states from empty states is the free electron Fermi surface at $0^{\circ} \mathrm{K}$.

In the real crystal the array of ions scatters the electrons producing Bragg reflections. This produces distortions of the free electron energy bands, especially on the Brillouin zone boundaries and at points where several zone boundaries intersect.

The free electron energy bands possess a great number of accidental degeneracies which are destroyed once the effects 
of the ionic pseudopotentials are included. Some intrinsic degeneracies persist however at points and along lines of special symmetry in $\mathrm{k}$ space. It is possible to make use of group theoretical arguments and other symmetxy considerations to predict the types of degeneracy which occur in mercury. In mercury there are two valence electrons per atom, which is sufficient to fill one complete Brillouin zone. In general. it is necessary to calculate the geometry of the Brillouin zone so as to be able to predict what kinds of distortions of the spherical free electron Fermi surface will be produced.

2. The Crystal Structure and Brillouin Zone of Mercury

Mercury crystallizes at approximately $223^{\circ} \mathrm{K}$ into a rhombohedral lattice at atmospheric pressure with one atom per unit cell.

It is customary to relate the three basis vectors to a fixed coordinate system so that each one is equally inclined to the $z$ axis at an angle $\theta$. In the diagram the $z$ axis is

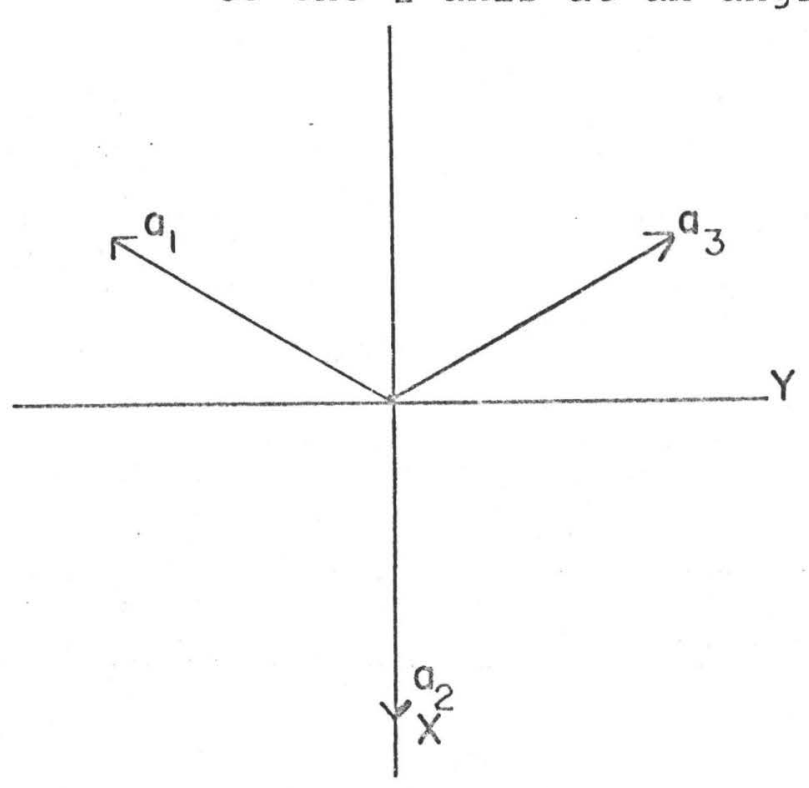
taken to be normal to the plane of the paper and the arrows show the projections of the primitive basis vectors on to the $x y-p l a n e$. The length of each vector is called the rhombohedral vector, and it is 
customary to refer to the angle between two vectors as the rhombohedral angle.

If the rhombohedral. vector is equal to $a_{0}$, then,

$$
\begin{aligned}
& \underline{a}_{1}=a_{0}\left(-\frac{\sin \theta}{2},-\sin \theta \frac{\sqrt{3}}{2}, \cos \theta\right) . \\
& \underline{a}_{2}=a_{0}(\sin \theta, \cos \theta) . \\
& \underline{a}_{3}=a_{0}\left(-\frac{\sin \theta}{2}, \sin \theta \frac{\sqrt{3}}{2}, \cos \theta\right) .
\end{aligned}
$$

By considering the inner product of two vectors, it follows that the rhombohedral angle a satisfies the relation.

$$
\sin \frac{\alpha}{2}=\frac{\sqrt{3}}{2} \sin \theta
$$

If the reciprocal lattice vectors $\underline{\mathrm{b}}_{1}, \underline{\mathrm{b}}_{2}, \underline{\mathrm{b}}_{3}$ are assumed to satisfy,

$$
\underline{a}_{i} \cdot \underline{b}_{j}=2 \pi \delta_{i j} \cdot
$$

Then

$$
\begin{aligned}
& \underline{b}_{1}=\frac{2 \pi}{a_{0}}\left(-\frac{1}{3} \frac{1}{\sin \theta},-\frac{1}{\sqrt{3}} \frac{1}{\sin \theta}, \frac{1}{3} \frac{1}{\cos \theta}\right) \\
& \underline{b}_{2}=\frac{2 \pi}{a_{0}}\left(\frac{2}{3} \frac{1}{\sin \theta}, 0, \frac{1}{3} \frac{1}{\cos \theta}\right) \\
& \underline{b}_{3}=\frac{2 \pi}{a_{0}}\left(-\frac{1}{3} \frac{1}{\sin \theta}, \frac{1}{\sqrt{3}} \frac{1}{\sin \theta}, \frac{1}{3} \frac{1}{\cos \theta}\right) .
\end{aligned}
$$

The rhombohedral angle for the reciprocal lattice, $\beta$, is related to the direct lattice rhombohedral angle, $\alpha$, by

$$
\sin \frac{\beta}{2}=\frac{1}{2 \sin \frac{\alpha}{2}}
$$

Figure (III.I) is a diagram of the first Brillouin zone. of mercury with points of special interest shown. Directions are indicated using the convention that ( $\mathrm{mn}$ ) represents a 
direction in reciprocal space, or a plane perpendicular to the ( $(m n)$ direction. Real space directions and planes normal to them are represented by [lmn]. Sets of equivalent directions will be written as $\{\ell m n\}$ in reciprocal space and as < $\ell m n>$ in real space.

The three orthogonal real space directions [111], [1] 2$]$, and [II0], specify the same directions as the corresponding

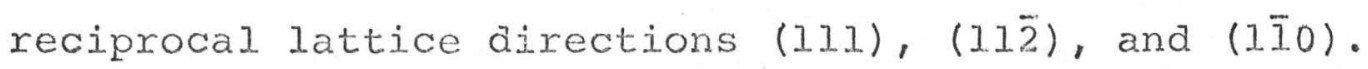

The Brillouin zone possesses the same point group symetry about the centre $\Gamma$ as the real lattice. The (111) direction is an axis of threefold symmetry and is referred to as the txigonal axis. The trigonal-bisectrix $\{1 \overline{1} 0\}$ planes which bisect the Brillouin zone along the line $\mathrm{T}-\mathrm{U}_{2}-\mathrm{X}_{1}-\mathrm{U}_{3}-\mathrm{I}_{2}$ are the mirror planes for mercury. There is an inversion symmetry about the centre $\Gamma$, and an axis of two-fold symmetry about the line (IIO) which is called the binary axis.

The smallest slice of the zone which will reproduce the entire Brillouinzone if these symmetry operations are applied to it is shown outlined in the figure. It is bounded by two mirror planes, the plane perpendicular to IT, and the planes forming the zone boundary. This section which is $1 / 12$ of the complete zone, will be referred to as the basic section in this thesis. It has $\mathrm{T} T$ along the positive $\mathrm{z}$ axis (1II), with IS' along the positive $x$ axis. The $y$ axis forms a right-handed set with the $x$ and $z$ axes. 


\section{Figure (III.])}

The Brillouin zone of mercury, and the

'basic section' of the zone

The diagram shows the Brillouin zone of mercury and some of the important symmetry points and directions.

$\Gamma$ is at the centre of the zone and is the origin of an orthogonal set of axes. THe $z$ axis lies along TT in the trigonal direction, $\mathrm{SS}^{\prime}$ defines the $\mathrm{X}$ axis which lies in the mixror plane $\mathrm{T}-\mathrm{U}_{1}-\mathrm{L}_{1}-\mathrm{S}^{\prime}-\mathrm{T}$. The $\mathrm{Y}$ axis is at $90^{\circ}$ to this plane.

The 'basic section' is equal to $\frac{1}{12}$ of the complete zone and is bounded by two mirror planes, the plane $\mathrm{z}=0$, and the zone boundary. The (111) face is a perfect hexagon with $\mathrm{T}$ at the centre, $\mathrm{U}_{1}$ and $\mathrm{U}_{2}$ bisect two of the edges of the hexagon. ${ }^{x}$. is at the centre of a rectangular face with $\mathrm{k}$ at the mid-moint of the edge $W_{1} W_{3} \cdot I_{1}$ is at the centre of the pseudo-hexagonal (010) face.

The point $K^{\prime}$ is equivalent to $K$, and $S^{\prime}$ is equivalent to $\mathrm{s}$. There are three bisectrix and three binary axes in the $\mathrm{z}=0$ plane. IS and $\Gamma \mathrm{S}^{\mathrm{a}}$ are both bisectrix axes, the (1̄0) binary axis is shown on the diagram, $\Gamma K^{\prime}$ is a.lso a binary axis. 


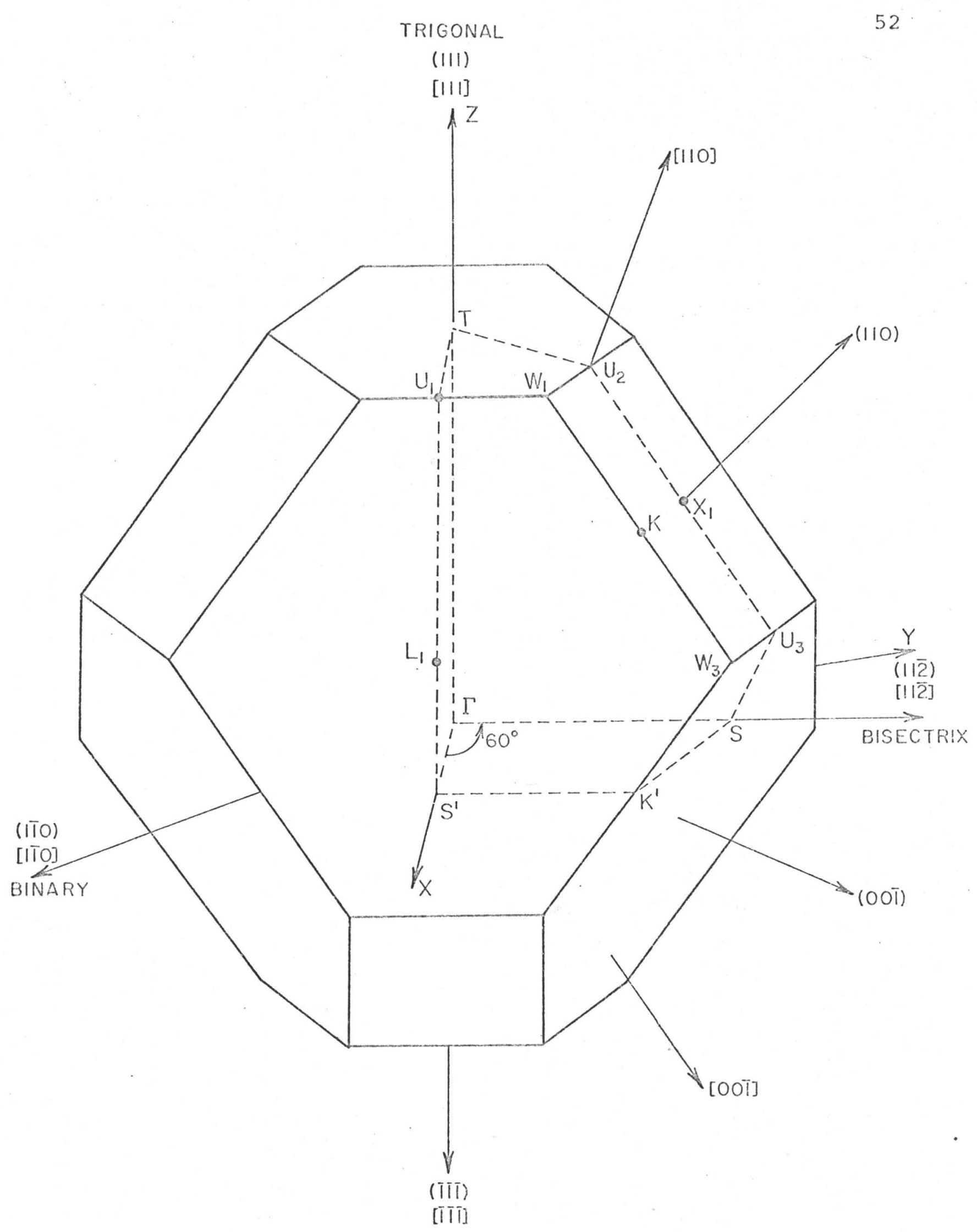


The points $\mathrm{U}_{1}, \mathrm{U}_{2}, \mathrm{U}_{3}$ etc, are really equivalent but have been labelled with a suffix for convenience.

Table (III.I) given by Brandt and Rayne (1966), lists some of the important crystallographic data for mercury.

\section{The Free Electron Model}

In a pseudopotential band structure calculation a secular equation is solved which has as its matrix elements,

$$
\begin{gathered}
\left\langle\underline{k}-\underline{G}_{I}\left|H_{p}\right| \underline{k}-G_{2}\right\rangle=\left|\underline{k}-G_{I}\right|^{2} \delta_{G_{I}, G_{2}}+\frac{l}{\Omega} U\left(\underline{G}_{2}-G_{I}\right) \\
+\frac{4 \pi}{\Omega} \sum_{n \ell \ell}\left(E_{1}-E_{n \ell}\right)(2 \ell+1) P_{\ell}\left(\cos \left(k-G_{I}, k-G_{2}\right)\right) \\
\times \int j_{\ell}\left(\left|k-G_{1}\right| r\right) r P_{n \ell}(r) d r \times \int j_{\ell}\left(\left|k-G_{2}\right| r\right) r P n \ell(r) d r .
\end{gathered}
$$

For any particular value of $k$ the set of eigenvalues $E_{i}(k)$ can be extracted and it is possible to construct graphs showing the variation of these energy bands along various lines in $\mathrm{k}$ space. The solutions of the secular equation will automatically be given in the repeated zone scheme.

In the free electron approximation the equation becomes diagonal with the eigenvalues given by

$$
E_{i}(k)=\left|\underline{k}-\underline{G}_{i}\right|^{2} .
$$

The free electron energy bands are thus given by the squares of the distances from the various points $\mathrm{k}$ to the different reciprocal lattice vectors. Figures (III.2) and (III.3) show the free electron bands computed for mercury along various Jines in the Brillouin zone. The free electron Fermi energy 
TABLE (IIT.1)

Crystallographic data for $\mathrm{Hg}$

\begin{tabular}{|c|c|c|c|}
\hline Symbol. & 1. Value & $\begin{array}{l}\text { Value } \\
\left(\text { units of } b_{0}\right) \\
\end{array}$ & Definition \\
\hline$a_{0}$ & $2.9863 \AA$ & & Rhombohedral vector at $5^{\circ} \mathrm{K}$ \\
\hline$\alpha$ & $70^{\circ} 44.6^{\prime}$ & & Rhombohedral angle at $5^{\circ} \mathrm{K}$ \\
\hline$b_{0}$ & $\begin{array}{l}1.0932\left(2 \pi / a_{0}\right) \\
\left(=2.3002 \AA^{-1}\right)\end{array}$ & 1.000 & Reciprocal lattice vector \\
\hline$\beta$ & $104.36^{\circ}$ & & $\begin{array}{l}\text { Rhombohedral angle for } \\
\text { reciprocal lattice }\end{array}$ \\
\hline$\theta^{\prime}$ & $65.80^{\circ}$ & & $\begin{array}{l}\text { Angle between }(100), b_{0} \text {, and } \\
\text { txigonal axis, (111) }\end{array}$ \\
\hline$\phi^{\prime}$ & $48.05^{\circ}$ & & $\begin{array}{l}\text { Angle between (110) and } \\
\text { (11]) }\end{array}$ \\
\hline $\mathrm{V}$ & $\begin{array}{l}0.8631 \mathrm{a}_{0}^{3} \\
\Leftrightarrow=22.986 \AA^{3}\end{array}$ & & Volume of unit cell. \\
\hline$\Gamma-\mathrm{L}$ & $1.1500 \AA^{-1}$ & 0.5000 & $\frac{1}{2}(100)$ \\
\hline$\Gamma-X$ & $1.4103 \AA^{-1}$ & 0.6131 & $\frac{1}{2}(110)$ \\
\hline$\Gamma-T$ & $1.4114 \AA^{-1}$ & 0.6149 & $\frac{1}{2}(111)$ \\
\hline $\mathrm{k}_{\mathrm{F}}$ & $1.3708 \AA^{-1}$ & 0.5960 & Fermi radius \\
\hline$E_{F^{\prime}}$ & 0.5240 Rya. & & Fermi energy (Free electron) \\
\hline$X-U$ & & 0.2363 & $\begin{array}{l}\frac{1}{2} \text { length of (110) face of } \\
\text { Brillouin zone }\end{array}$ \\
\hline $\mathrm{T}-\mathrm{U}$ & & 0.2719 & $\begin{array}{l}\frac{1}{2} \text { width of (1ll) face of } \\
\text { Brillouin zone }\end{array}$ \\
\hline$I-U$ & & 0.4494 & \\
\hline$\left\{\begin{array}{l}X-K \\
U-W\end{array}\right.$ & & 0.1570 & $\begin{array}{l}\frac{1}{2} \text { width of }(110) \text { face of } \\
\text { Brillouin zone }\end{array}$ \\
\hline $\mathrm{T}-\mathrm{W}$ & & 0.3139 & \\
\hline
\end{tabular}




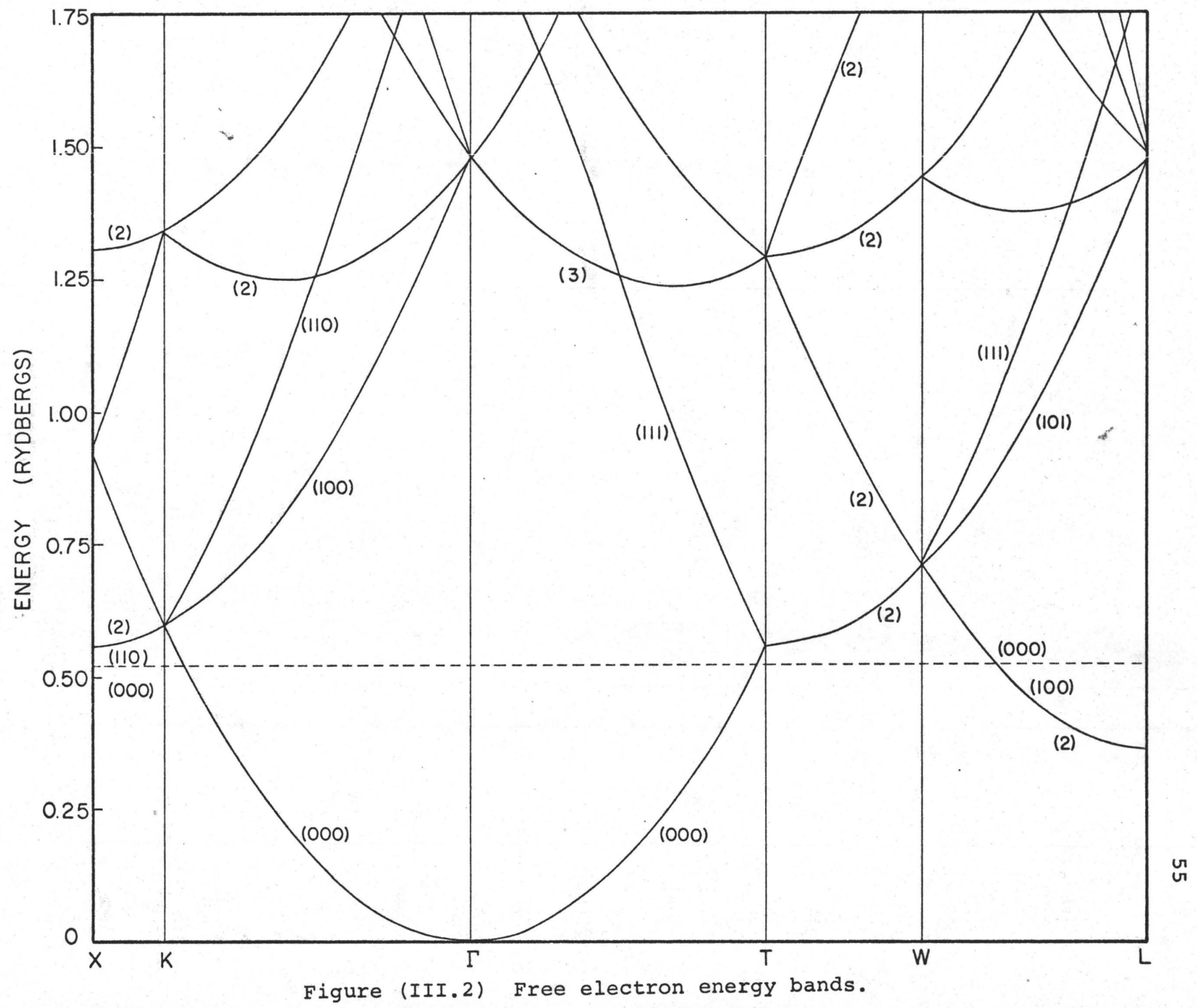




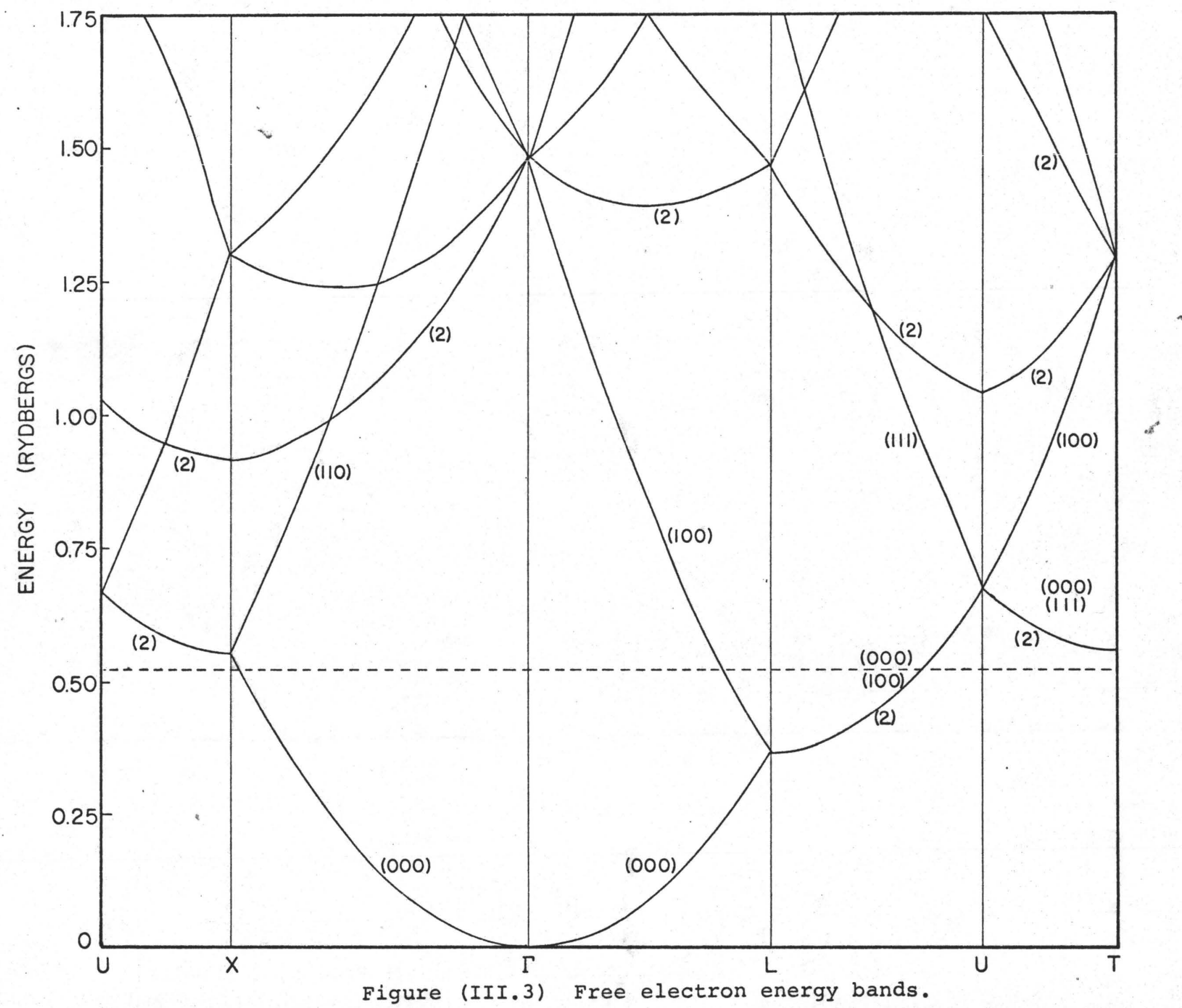


is indicated by the dotted line at 0.5240 Rydbergs. Some of the degeneracies are indicated by small numerals on the graph. These degeneracies do not include the extra degeneracy of 2 due to the electron spin.

Figures (III.4) and (III.5) show the electron bands computed using a local pseudopotential. The pseudopotential used is that of Brandt and Rayne (1966) in which the matrix elements are given by the formula

$$
\left\langle\underline{k}-\underline{G}_{1}\left|H_{p}\right| \underline{k}-G_{2}\right\rangle=\left|\underline{k}-\underline{G}_{2}\right|^{2} \delta_{G_{1} G_{2}}+W\left(\underline{G}_{2}-G_{1}\right) .
$$

In Brandt and Rayne's local pseudopotential all the Fourier components $W(\underline{G})$ are zexo except for those corresponding to the $L, X$, and $T$ faces of the Brillouin zone $W_{L}, W_{X}$, and $W_{T}$ respectively, which have the values

$$
\begin{aligned}
& \mathrm{W}_{\mathrm{L}}=\mathrm{W}_{100}=-0.066 \text { Rydbergs. } \\
& \mathrm{W}_{\mathrm{X}}=\mathrm{W}_{110}=0.047 \text { Rydbergs. } \\
& \mathrm{W}_{\mathrm{T}}=\mathrm{W}_{1 \mathrm{II}}=0.047 \text { Rydbergs. }
\end{aligned}
$$

A comparison of these bands with the free electron bands shows there is a great deal of similarity, but the pseudopotential has destroyed the accidental degeneracies of the free electron model. The degeneracies that remain are intrinsic and are a consequence of the crystal structure. Group theory and simple perturbation theory can be used to calculate approximate values of the energy gaps and the energy level sequence produced by a weak pseudopotential. 


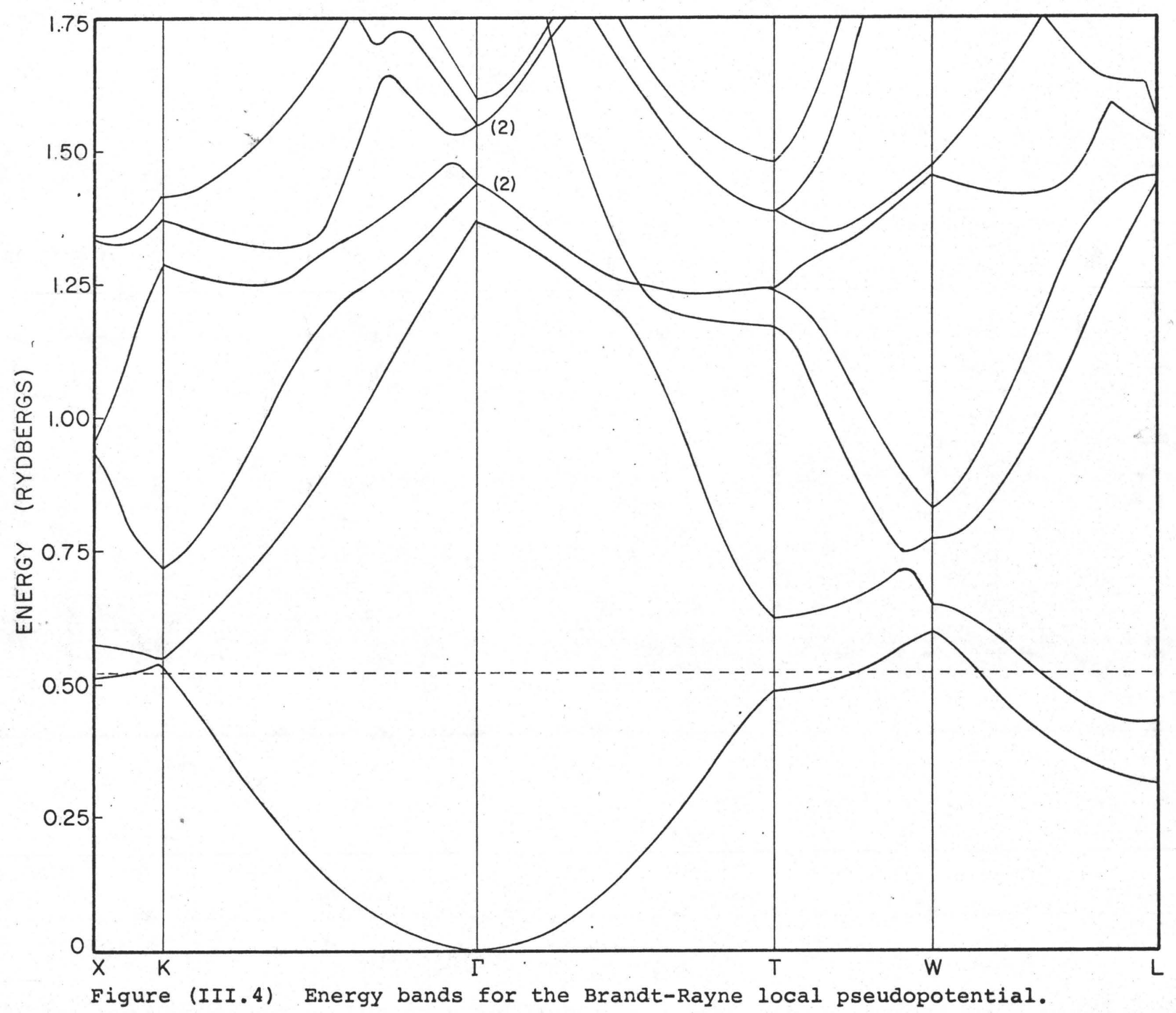




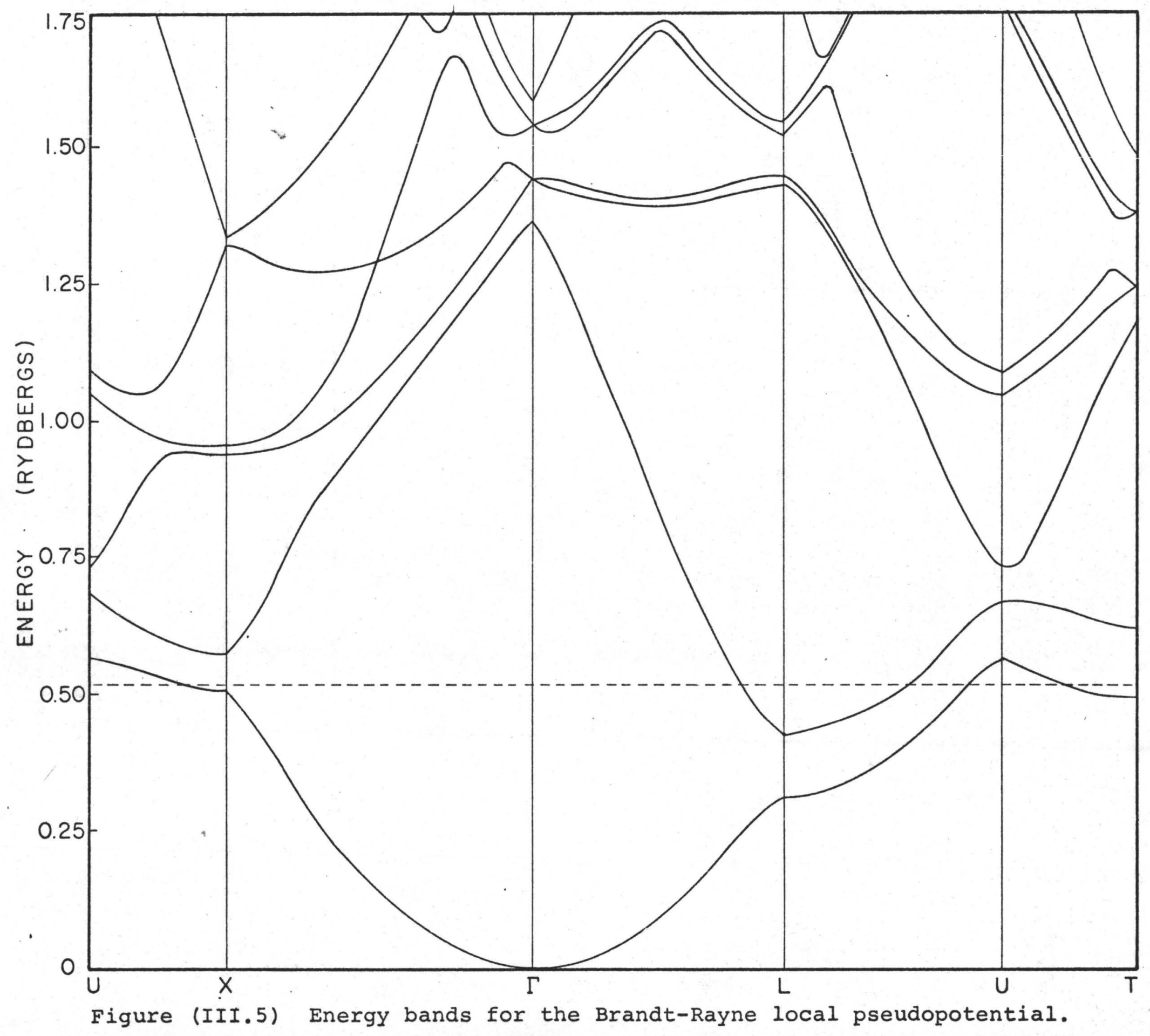


At a general point in the Brillouin zone, first order perturbation theory gives the energy of the lowest band as

$$
\mathrm{E}_{\mathrm{k}}=\mathrm{k}^{2}+\left\langle\mathrm{k}\left|\mathrm{w}_{\mathrm{p}}\right| \mathrm{k}\right\rangle
$$

If the notation is adopted that

$$
w_{p}\left(k_{1}, k_{2}\right)=\left\langle k_{1}\left|w_{p}\right| k_{2}\right\rangle
$$

then

$$
\mathrm{E}_{\mathrm{k}}=\mathrm{k}^{2}+\mathrm{w}_{\mathrm{p}}(\mathrm{k}, \mathrm{k}) .
$$

$\mathrm{W}_{\mathrm{p}}$ can be written in the form of a sum of operators

$$
W_{p}=W_{L O C}+W^{(s)}+W^{(p)}+W^{(d)}+\ldots
$$

in which $W_{L O C}$ is the local part of the operator and the suffixes s, p, d refex to the different angular momentum components of the non local part.

This gives

$$
\begin{aligned}
& W_{p}\left(k_{1} k_{2}\right)=W_{L O C}\left(k_{1}-k_{2}\right)+W^{(s)}\left(k_{1}, k_{2}\right) \\
& +W^{(p)}\left(\underline{k}_{1}, \underline{k}_{2}\right)+W^{(d)}\left(\underline{k}_{1}, \underline{k}_{2}\right)+\ldots
\end{aligned}
$$

But $\quad W^{(s)}\left(k_{-1}, k_{2}\right)=I_{S}\left(k_{I}\right) I_{S}\left(k_{2}\right)$

$$
\begin{aligned}
& W^{(p)}\left(\underline{k}_{1}, \underline{k}_{2}\right)=I_{p}\left(k_{1}\right) I_{p}\left(k_{2}\right) \cos \left(\underline{k}_{1}, k_{2}\right) \\
& W^{(d)}\left(\underline{k}_{1}, \underline{k}_{2}\right)=I_{d}\left(k_{1}\right) I_{d}\left(k_{2}\right)\left(\frac{3}{2} \cos ^{2}\left(\underline{k}_{1}, k_{2}\right)-\frac{1}{2}\right)
\end{aligned}
$$

where the factors $I_{s}(k), I_{p}(k), I_{d}(k)$ depend on $I_{y}$ on the modulus of the vector $k$.

Substituting into equation (3.6)

$$
E_{k}=k^{2}+W_{J O C}(0)+I_{S}^{2}(k)+I_{p}^{2}(k)+I_{d}^{2}(k)
$$

If a purely local pseudopotential is being used 


$$
E_{k}=k^{2}+W_{L O C}(0) \text {. }
$$

This means that over most of the Brillouin zone the energy levels will strongly resemble the free electron bands except for a constant shift in energy equal to $\mathrm{w}_{\text {LoC }}(0)$.

The preceding treatment will be inadequate at points in k space that are at almost equal distances from more than one reciprocal lattice vector. Points that lie on the faces of the first Brillouin zone are equally close to two reciprocal lattice vectors, points such as $U$ and $K$ which lie on an edge are equally near three, and vertex points such as $W$ are equidistant from four reciprocal lattice vectors. These points need special treatment.

\section{The Energy Gap at a General Point on a Zone Face}

The simplest approximation that can be used at a point that is equally close to two reciprocal lattice points is to diagonalize a $(2 \times 2)$ secular equation.

$$
\text { If }\left|k_{1}\right\rangle=|k\rangle \text {, and }\left|k_{2}\right\rangle=|k-\underline{G}\rangle
$$

and

$$
\left|k_{1}\right|=\left|k_{2}\right|=k
$$

then the $(2 \times 2)$ secular equation is

$$
\left|\begin{array}{cc}
\left.\left[k^{2}+<k_{1}\left|w_{p}\right| k_{1}\right\rangle-\lambda\right] & \left.<k_{1}\left|w_{p}\right| k_{2}\right\rangle \\
\left.<k_{2}\left|w_{p}\right| k_{1}\right\rangle & \left.\left[k^{2}+<k_{2}\left|w_{p}\right| k_{2}\right\rangle-\lambda\right]
\end{array}\right|=0
$$

This equation has an eigenstate of even symmetry

$$
\left|\psi_{+}\right\rangle=\frac{1}{\sqrt{2}}\left(\left|k_{1}\right\rangle+\left|k_{2}\right\rangle\right) ;
$$


with energy equal to

$$
E_{+}=k^{2}+w_{p}\left(k_{1}, k_{2}\right)+w_{p}\left(k_{1}, k_{1}\right) \text {. }
$$

And another eigenstate of odd symmetry

$$
\left|\psi_{-}\right\rangle=\frac{1}{\sqrt{2}}\left(\left|\cdot k_{1}\right\rangle-\left|\cdot k_{2}\right\rangle\right),
$$

with energy equal to

$$
E_{-}=k^{2}-w_{p}\left(k_{1}, k_{2}\right)+w_{p}\left(k_{1}, k_{1}\right)
$$

The wave-function

$$
\left|\psi_{+}\right\rangle=\sqrt{\frac{2}{V}} e^{i\left(\underline{k}-\frac{G}{2}\right) \cdot \underline{r}} \cos \left(\frac{G}{2} \cdot \underline{r}\right)
$$

has some similarity to an atomic s-like wave function, whilst

$$
\left|\psi_{-}\right\rangle=\sqrt{\frac{2}{V}} e^{i\left(\underline{k}-\frac{G}{2}\right) \cdot \underline{r}} \sin \left(\frac{G}{2} \cdot \underline{r}\right)
$$

resembles an atomic p-like wave function.

The energies can also be written as

$$
\begin{gathered}
E_{+}=k^{2}+W_{L O C}(0)+W_{L O C}(G)+2 I_{S}^{2}(k) \\
+I_{p}^{2}(k)\left(I+\cos \left(k_{1}, k_{2}\right)\right)+I_{d}^{2}(k)\left(\frac{3}{2} \cos ^{2}\left(k_{1}, k_{2}\right)+\frac{1}{2}\right)
\end{gathered}
$$

and

$$
\begin{gathered}
E_{-}=k^{2}+W_{L O C}(0)-W_{L O C}(G)+I_{p}^{2}(k)\left(1-\cos \left(k_{1}, k_{2}\right)\right) \\
+I_{d}^{2}\left(\frac{3}{2}-\frac{3}{2} \cos ^{2}\left(k_{1}, k_{2}\right)\right) .
\end{gathered}
$$

The fact that $W_{\text {Loc }}(0)$ is present in both (3.13) and (3.14) is because $W_{\text {LOC }}(0)$ is always present as a constant term on the diagonal elements of a pseudopotential secular equation, it shifts all the energy bands by a constant amount 
and has no effect upon the band structure.

At points such as $L, X$, and $T$ on the zone faces the vectors $\underline{k}_{1}$ and $\underline{k}_{2}$ are equal and opposite, giving $\cos \left(k_{1}, k_{2}\right)=-1$.

At such points

$$
E_{+}=k^{2}+W_{L O C}(0)+W_{L O C}(G)+2 I_{S}^{2}(k)+2 I_{d}^{2}(k)
$$

and

$$
E_{-}=k^{2}+W_{L O C}(0)-W_{L O C}(\underline{G})+2 I_{p}^{2}(k) \text {. }
$$

From equations (3.13) and (3.14) the energy gap on the zone face is given by

$$
\begin{aligned}
\Delta E=E_{+}-E_{-}= & 2 W_{L O C}(G)+2 I_{s}^{2}(k)+2 I_{p}^{2}(k) \cos \left(k_{1}, k_{2}\right) \\
& +2 I_{d}^{2}(k)\left(\frac{3}{2} \cos ^{2}\left(k_{1} k_{2}\right)-\frac{1}{2}\right)
\end{aligned}
$$

The various terms in equation (3.16) do not vary very much over quite a large area around the centres of the zone faces. There is a tendency for the two lowest bands to run parallel in these regions.

\section{Group Theoretical Considerations}

The pseudopotential splits most of the accidental degeneracies of the free electron model, howewer there are still some doubly degenerate energy levels along the trigonal axis. On this axis some of the levels are singlets and others are doublets. This is a consequence of the symmetry of the rhombohedral lattice.

At the points $\Gamma$ and $T$ the 'group of $k^{\prime}$ is the same as 
the point group of the crystal $3 \mathrm{~m}$ (or $\mathrm{D}_{3 \mathrm{~d}}$ ). This group consists of 12 operations divided up into 6 classes.

$$
\begin{gathered}
\mathrm{E} \\
\mathrm{C}_{3}, \mathrm{C}_{3}^{-1} \\
\mathrm{~m}_{1}, \mathrm{~m}_{2}, \mathrm{~m}_{3} \\
\mathrm{~J} \\
\mathrm{JC}_{3}, \mathrm{JC}_{3}^{-1} \\
3 \mathrm{C}_{2}=\mathrm{J}_{\mathrm{m}_{1}}, \mathrm{~J}_{\mathrm{m}_{2}}, \mathrm{~J}_{\mathrm{m}_{3}}
\end{gathered}
$$

The identity

Rotations of $120^{\circ}$ and $210^{\circ}$ about the trigonal axis.

Three reflection planes passing through the trigonal axis.

The inversion.

Two rotation inversions by $120^{\circ}$ and $240^{\circ}$

Three two-fold rotations about axes perpendicular to the trigonal axis.

The character table of this group has been given by Jones

(H. Jones, The theory of Brillouin zones and electronic states in crystals, p. 95).

TABLE III. 2

The character table for the group $\overline{3} \mathrm{~m}$

\begin{tabular}{ccccccc}
\hline & $E$ & $2 \mathrm{C}_{3}$ & $3 \mathrm{~m}$ & $\mathrm{~J}$ & $2 \mathrm{JC}_{3}$ & $3 \mathrm{C}_{2}$ \\
\hline$\Gamma_{1}^{+}$ & 1 & 1 & 1 & 1 & 1 & 1 \\
$\Gamma_{2}^{+}$ & 1 & 1 & -1 & 1 & 1 & -1 \\
$\Gamma_{3}^{+}$ & 2 & -1 & 0 & 2 & -1 & 0 \\
$\Gamma_{1}^{-}$ & 1 & 1 & 1 & -1 & -1 & -1 \\
$\Gamma_{2}^{-}$ & 1 & 1 & -1 & -1 & -1 & 1 \\
$\Gamma_{3}^{-}$ & 2 & -1 & 0 & -2 & 1 & 0 \\
\hline
\end{tabular}


This table shows that the energy levels at $\Gamma$ and $T$ (neglecting the spin degeneracy) are singly or doubly degenerate.

At a general point on the trigonal axis the invexsion is not a member of the group and the symmetry belongs to the group $3 \mathrm{~m}\left(\right.$ or $\left.\mathrm{D}_{3}\right)$. This group consists of six operations divided into three classes, consequently there are three irxeducible representations, two of them are one dimensional and the other is two dimensional. This means that at a general point on the trigonal axis the energy levels are also singly or doubly degenerate.

It is possible that the irreducible representations of $\overline{3} \mathrm{~m}$ might become reducible under the lower symmetry of the group $3 \mathrm{~m}$. However this is not the case and it can be shown that the degeneracies remain constant as $\mathrm{k}$ increases from zero along the trigonal axis.

At points which do not lie on the trigonal axis, all the energy levels are singly degenerate (except for a spin degeneracy).

In the free elcctron approximation the second energy level at $\Gamma$ is a six-fold degenerate level at an energy $E_{k}=1.4820$ Rydbergs. This six fold acdidental degeneracy arises from the $\{100\}$ set of reciprocal lattice vectors. The corresponding set of plane waves may be written as $\left|g_{1}\right\rangle$ corresponding to $(100),\left|\bar{g}_{1}\right\rangle$ corresponding to $(\overline{1} 00)$ $\left|g_{2}\right\rangle$ corresponding to $(010),\left|\bar{g}_{2}\right\rangle$ corresponding to $(0 \bar{I} 0)$ $\left|g_{3}\right\rangle$ corresponding to $(001),\left|\bar{g}_{3}\right\rangle$ corresponding to $(00 \bar{I})$. 
In the presence of a pseudopotential this six-fold accidental degeneracy will split up into two singlets and two doublets. If suitable linear combinations of these plane waves are taken the $(6 \times 6)$ secular equation will diagonalize and first order perturbation theory can be used to estimate the energy levels. Some theorems of group theory show how this can be done.

One theorem (Tinkham, Group Theory and Quantum Mechanics, p. 80) states that the matrix elements of an operator $\mathrm{H}$ which is invariant under all the operations of a group will vanish between functions belonging to different unitary representations or to different rows of the same unitary representation. Another theorem (Tinkham, Theorem (3-33), p. 40) states that any function $F$ which is in the space operated on by a group of transformations $\mathrm{P}_{\mathrm{R}}$ can be uniquely decomposed into a sum of the form,

where $f_{K}^{(j)}$ belongs to the $k^{\text {th }}$ row of the $j^{\text {th }}$ irreducible

$$
F=\sum_{j=1}^{c} \sum_{k=1}^{\ell} f_{k}^{(j)},
$$
representation.

There is a set of projection operators which can be used to effect this decomposition. The most general operator is given by,

$$
P_{\lambda K}^{(j)}=\frac{{ }_{j}}{h} \sum_{R} \Gamma{ }^{(j)}(R){ }_{\lambda K}^{*}{ }^{P} R
$$

where $j$ specifies the representation which is of dimensionality $l_{j}, h$ is the number of group elements $I^{(j)}(R){ }_{\lambda k}$ is the 
$(\lambda, k)^{\text {th }}$ element of the unitary matrix that represents the group element $R$, the transformation operators $P_{R}$ are defined in the sense that $P_{R} f(\underline{x})=f\left(R^{-1} \underline{x}\right)$; where $R$ is a real orthogonal transformation of the coordinates that corresponds to one of the geometrical group operations.

The application of $\mathrm{P}_{\lambda \mathrm{K}}^{(j)}$ to an arbitrary function $\mathrm{F}$ will yield zero unless $F$ has a component belonging to the $k^{\text {th }}$ row of $\Gamma(j)$ in which case the transformed function will belong to the $\lambda^{\text {th }}$ row of $\Gamma^{(j)}$. If the function $\phi_{K}^{(j)}$ belongs to the $k^{\text {th }}$ row of $\Gamma^{(j)}$ then,

$$
P_{K K}^{(j)} \phi_{K}^{(j)}=\phi_{K}^{(j)}
$$

so that $\mathrm{P}_{K K}^{(j)}$ is a typical idempotent projection operator.

Another more general projection operator can be defined where,

$$
P^{(j)}=\sum_{K} P_{K K}(j)=\frac{l j}{h} \sum_{R} X^{(j)}(R){ }^{*} P_{R}
$$

This operator simply picks out that part of a function which belongs to the $j^{\text {th }}$ representation. It is a convenient form to use because it only requires a knowledge of the group characters $x^{(j)}(R)$.

This operator can be used to find the linear combinations of plane waves which will diagonalize the $(6 \times 6)$ secular equation. Selecting one of the plane waves and applying the group transformation operators in turn gives, $P(E)\left|g_{1}\right\rangle=\left|g_{1}\right\rangle ; P\left(C_{3}\right)\left|g_{1}\right\rangle=\left|g_{2}\right\rangle ; P\left(C_{3}^{-1}\right)\left|g_{1}\right\rangle=\left|g_{3}\right\rangle$ 


$$
\begin{aligned}
& \mathrm{P}\left(\mathrm{m}_{1}\right)\left|g_{1}\right\rangle=\left|g_{3}\right\rangle ; \mathrm{P}\left(\mathrm{m}_{2}\right)\left|g_{1}\right\rangle=\left|g_{1}\right\rangle ; \mathrm{P}\left(\mathrm{m}_{3}\right)\left|g_{1}\right\rangle=\left|g_{2}\right\rangle \\
& \mathrm{P}(\mathrm{J})\left|g_{1}\right\rangle=\left|\bar{g}_{1}\right\rangle ; \mathrm{P}\left(\mathrm{JC}_{3}\right)\left|g_{1}\right\rangle=\left|\bar{g}_{2}\right\rangle ; \mathrm{P}\left(\mathrm{JC}_{3}^{-1}\right)\left|g_{1}\right\rangle=\left|\bar{g}_{3}\right\rangle \\
& \mathrm{P}\left(\mathrm{J}_{\mathrm{m}_{1}}\right)\left|g_{1}\right\rangle=\left|\bar{g}_{3}\right\rangle ; \mathrm{P}\left(\mathrm{J}_{\mathrm{m}_{2}}\right)\left|g_{1}\right\rangle=\left|\bar{g}_{1}\right\rangle ; \mathrm{P}\left(\mathrm{J}_{\mathrm{m}_{3}}\right)\left|g_{1}\right\rangle=\left|\bar{g}_{2}\right\rangle
\end{aligned}
$$

Taking each irreducible representation in turn for the group ( $\overline{3} \mathrm{~m})$, using the group characters given in Table (III.2). For the $\Gamma_{1}^{+}$representation this gives,

$$
\begin{aligned}
& P^{\left(\Gamma_{1}^{+}\right)} \mid g_{1}>=\frac{1}{12}\left[P(E)\left|g_{1}>+P\left(C_{3}\right)\right| g_{1}>+P\left(C_{3}^{-1}\right) \mid g_{1}>\right. \\
& +P\left(m_{1}\right)\left|g_{1}>+P\left(m_{2}\right)\right| g_{1}>+P\left(m_{3}\right)\left|g_{1}>+P(J)\right| g_{1}> \\
& +P\left(J C_{3}\right)\left|g_{1}>+P\left(J_{3}^{-1}\right)\right| g_{1}>+P\left(J_{m_{1}}\right) \mid g_{1}> \\
& \left.+P\left(J_{m_{2}}\right)\left|g_{1}>+P\left(J_{m_{3}}\right)\right| g_{1}>\right] .
\end{aligned}
$$

which reduces to,

$$
\mathrm{P}^{\Gamma^{+}}\left|g_{1}\right\rangle=\frac{1}{6}\left[\left|g_{1}\right\rangle+\left|g_{2}\right\rangle+\left|g_{3}\right\rangle+\left|\bar{g}_{1}\right\rangle+\left|\bar{g}_{2}\right\rangle+\left|\bar{g}_{3}\right\rangle\right] \text {. }
$$

In a similar way it can be shown that,

$$
\begin{aligned}
& \mathrm{P}^{\Gamma^{+}}{ }^{+} \mathrm{g}_{1}>=0 \\
& \mathrm{P}^{\Gamma^{+}}{ }^{+}\left|g_{1}\right\rangle=\frac{1}{6}\left[2\left|g_{1}\right\rangle-\left|g_{2}\right\rangle-\left|g_{3}\right\rangle+2\left|\bar{g}_{1}\right\rangle-\left|\bar{g}_{2}\right\rangle-\left|\bar{g}_{3}\right\rangle\right] . \\
& \mathrm{P}^{\Gamma^{-}}\left|g_{1}\right\rangle=\frac{1}{6}\left[\left|g_{1}\right\rangle+\left|g_{2}\right\rangle+\left|g_{3}\right\rangle-\left|\bar{g}_{1}\right\rangle-\left|\bar{g}_{2}\right\rangle-\left|\bar{g}_{3}\right\rangle\right] \text {. } \\
& P^{\Gamma^{-}}\left|g_{1}\right\rangle=0 \\
& P^{\Gamma^{-}}\left|g_{1}\right\rangle=\frac{1}{6}\left[2\left|g_{1}\right\rangle-\left|g_{2}\right\rangle-\left|g_{3}\right\rangle-2\left|\bar{g}_{1}\right\rangle+\left|\bar{g}_{2}\right\rangle+\left|\bar{g}_{3}\right\rangle\right] \text {. }
\end{aligned}
$$

With these linear combinations of plane waves as basis functions (suitably normalized) the equation will diagonalize 
sufficiently so that first order perturbation theory can be used. Thus the $\Gamma_{1}^{+}$linear combination will form a singlet with energy given by

$$
\left.\mathrm{E}_{\Gamma_{1}^{+}}=\mathrm{E}_{\mathrm{k}}+\frac{1}{6}<g_{1}+g_{2}+g_{3}+\bar{g}_{1}+\bar{g}_{2}+\bar{g}_{3}\left|w_{p}\right| g_{1}+g_{2}+g_{3}+\bar{g}_{1}+\bar{g}_{2}+\bar{g}_{3}\right\rangle,
$$

which reduces to,

$$
\mathrm{E}_{\Gamma_{1}^{+}}=\mathrm{E}_{\mathrm{k}}+\mathrm{W}\{000\}+2 \mathrm{~W}\{110\}+2 \mathrm{~W}\{10 \bar{I}\}+\mathrm{W}\{002\} .
$$

The other representations may be treated in exactly the same way and the predicted energy levels are

$\Gamma_{1}^{+}$singlet, $E=1.4820+W(000)+2 W\{110\}+2 W\{10 \bar{I}\}+W\{002\}$

$\Gamma_{3}^{+}$doublet, $E=1.4820+W(000)-W\{110\}-W\{10 \bar{I}\}+W\{002\}$

$\Gamma_{I}^{-}$singlet, $\mathrm{E}=1.4820+W(000)-2 W\{110\}+2 W\{10 \bar{I}\}-W\{002\}$

$\Gamma_{3}^{-}$doublet, $E=1.4820+W(000)+W\{110\}-W\{10 \bar{I}\}-W\{002\}$.

The energy bands shown in Figures (III.4) and (III.5) were computed using Brandt and Rayne's local pseudopotential for which $W\{110\}=0.047$ Rydbergs, $W\{10 \bar{I}\}=0, W\{002\}=0$. The value of $W\{000\}$ is irrelevant and has been taken to be zero. The following table compares the energies predicted using the simple formulae of equations (3.19) with the energies calculated by diagonalizing a $(15 \times 15)$ secular equation for this pseudopotential.

Energy level Energy (perturbation theory) Energy (secular equations)
$\Gamma_{1}^{+}$singlet
1.576
1.597 singlet
$\Gamma_{3}^{-}$doublet
1.529
1.552 doublet 
$\Gamma_{3}^{+}$doublet

$\Gamma_{i}^{-}$singlet
1.435

1.388
1.443 doublet

1.372 singlet.

In the previous table all the energies are in Rydbergs, It is clear that the simple perturbation theory formulae are very useful for providing insight into the nature of the local pseudopotential energy bands.

6. The Effect of Spin-orbit Coupling

In the simple theory of metals it is usual to regard each state in the Brillouin zone as doubly degenerate with respect to spin. If spin-orbit coupling is included this simple description may not be correct. However it can be shown (H. Jones, The theory of Brillouin zones and electronic states in crystals, p. 262) that the energy levels will still be doubly degenerate if the crystal has inversion symmetry.

The spin-orbit Hamiltonian

$$
H=\frac{p^{2}}{2 m}+V(\underline{r})+\frac{K}{4 m^{2} c^{2}}(\nabla V \times \underline{p} \cdot \underline{\sigma})
$$

has the property that, $\sigma_{Y} H^{*} \sigma_{Y}^{-1}=H_{i}$ where $\sigma_{Y}=\left(\begin{array}{cc}0 & -i \\ i & 0\end{array}\right)$ is the Pauli spin operator. This means that if $\psi(\underline{r})$ is a two component wave-function of energy $\mathrm{E}$, so also is $\sigma_{\mathrm{Y}} \psi^{*}(r)$. However the two components of $\psi_{1} \psi_{1}$ and $\psi_{2}$ are both Bloch

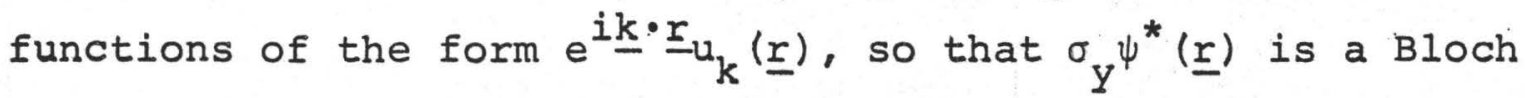
function having wave number $-\underline{k}$. This gives the result that the energy bands possess inversion symmetry and $E_{\uparrow}(\underline{k})=E_{\downarrow}(-\underline{k})$. This result, which is a consequence of the time reversal symmetry 
of the Schrödinger equation is true irrespective of whether the crystal itself has inversion symmetry.

If the Schrödinger equation is written out in full and solved for the periodic parts of the wave function $u_{\underline{k}}(\underline{\underline{r}})$, the resulting equation contains terms of the form,

$$
\nabla^{2} u+2 \underline{i k} \cdot \nabla u+\frac{2 m}{\hbar^{2}}\left(E-\frac{\hbar^{2} k^{2}}{2 m}-v(\underline{r})\right) u ;
$$

together with other terms from the spin-orbit part of the Hamiltonian. This equation has the property that if $\underline{\mathrm{k}}$ is replaced by $-\underline{k}$ and an inversion is carried out so that $\underline{r}$ is transformed to $\underline{-r}$, then the equation will remain invariant if and only if $\mathrm{V}(\underline{\underline{r}})=\mathrm{V}(\underline{\underline{r}})$. If this is true it means that $u_{\underline{\underline{k}}}(\underline{r})=\underline{u}_{\underline{k}}(\underline{-\underline{r}})$, so that $\underline{u}_{\underline{k}}(\underline{r})$ and $\underline{u}_{\underline{k}}(-\underline{r})$ which are two different functions belong to the same energy.

This establishes the important result that there is a twofold degeneracy at a general point in the Brillouin zone if the crystal has an inversion centre. This means that every energy level in mercury is at least twofold degenerate.

On the trigonal axis some of the orbital levels belonging to $\Gamma^{3}$ are already doubly degenerate. This means that they are really fourfold degenerate when spin is taken into account. Spin-orbit couplig could split these levels into a pair of twofold levels.

To determine the types of degeneracy that occur when spin-orbit coupling is included it is necessary to make use of the double group. If the direct product representation $\Gamma(j) \times D(1 / 2)$ 
is reducible under the operations of the appropriate double group, then orbital degeneracies belonging to $\Gamma^{(j)}$ may be partially or completely lifted. .

Falicov and Golin (1965) have carried out this type of analysis for the symmetry points of. the Brillouin zone of arsenic. Mercury has the same type of Brillouin zone so the results for the arsenic structure can be applied to mercury. The most important physical result is that the orbital twofold degeneracies that occur on the trigonal axis are split.

Mercury is a heavy element and spin-orbit coupling is important. This means that all the energy levels of mercury are doubly degenerate, no other degeneracies occur. In mercury the only degeneracies that are lifted by the spinorbit effect are at energies well above the Fermi level. Spin-orbit coupling must influence the precise shape of the Fermi surface of mercury but it is difficult to separate these effects from the effects of a pseudopotential. 


\section{CHAPTER IV}

\section{THE METHOD OF THE PSEUDOPOTENTIAL CALCULATIONS}

The work described in this chapter was an attempt to find a pseudopotential that would give a better description of the energy bands and a better fit to the observed Fermi surface of mercury than had hitherto been given by the pseudo potentials reported by other workers.

\section{The Fermi Surface of Mexcury}

Previous experimental and theoretical investigations indicate that the Fermi surface of mercury is divided into two main sheets. In the first zone there is a multiply connected 'hole surface' which makes contact with the zone faces around the $\mathrm{X}$ and $\mathrm{T}$, the areas of contact apparently being quite small. The region of contact on the $I$ faces is much bigger. It is possible that the area is so large that it overlaps on to the $\mathrm{X}$ faces to a small extent. These contact regions are approximately circular on the $T$ faces, elliptical on the $\mathrm{X}$ faces, while the L face contact area is a large 'distorted circle'. The symmetry of the zone demands that the elliptical areas on the $X$ faces must have their semi-major axes in the direction $\mathrm{XU}$ and their semiminor axes in the direction $\mathrm{XK}$.

In the extended zone representation there are 
narrow cylindrical sections of the first zone surface which are located near the points $\mathrm{K}$ on the zone boundary with their axes parallel to the long edges of the $\mathrm{X}$ faces, so that their orientation is in the <100> direction. This feature of the Fermi surface of mercury is referred to as the $\beta$ arms. In the second zone there are lens shaped pieces of Fermi surface which are located on the L faces, their centres are at $L$ and their maximum cross sections are either in the L faces or are in planes that are tilted from these faces by a very small amount, perhaps a few degrees. As a rough approximation the lenses can be regarded as ellipsoids with two almost equal orthogonal axes along the LW and LU directions, with a much shorter axis at right angles to the other two and directed along the LT line. The lenses have inversion symmetry about $\mathrm{L}$ and they also possess a mirror symmetry because they are bisected by the (II0) mirror planes.

The first zone surface is topologically complex and it supports a large number of different types of closed and open orbits. The second zone lenses are geometrically simple and support the a orbits which are observed in de Haas van Alphen and Azbel--Kaner cyclotron resonance experiments.

This model of the Fermi surface has been deduced from a number of different experiments which have been carried out on oriented crystal of mercury. De Haas van Alphen effect experiments have been reported by Brandt and Rayne (1966), by 
Poulsen, Moss, and Datars (1970), and by Poulsen (1970). Datars and Dixon (1967) and Dishman and Rayne (1968) have interpreted their magnetoresistance data in terms of sets of open orbits whose existence is compatible with the topological model of the hole surface provided certain constraints are placed on the possible sizes of the breakthrough regions on the zone faces. Other experiments are the Azbel-Kaner cyclotron resonance experiments of Dixon and Datars (1968), and of Poulsen (1970). The magnetoacoustic attenuation experiments of Bogle, Coon, and Grenier (1969) have provided information on some of the important 'extremal calipers' of the Fermi surface.

2. The General Approach of the Pseudopotential Calculations It is characteristic of most pseudopotential calculations that the pseudohamiltonian contains a number of parameters whose values are not known in advance. The usual procedure is to select some experimental data and to vary the parameters until the best fit possible has been achieved. Once this has been done it is then possible to calculate other physical variables and to compare them with experiment.

The most accurate data relating to the dimensions of the Fermi surface of mercury that was available during the time that this research was being carried out were the de Haas . van Alphen effect measurements of Poulsen (1970). 
Poulsen's experiments provide accurate information (to within $2 \%$ ) about many of the extremal cross sectional areas of the Fermi surface for various orientations of the magnetic field in the trigonal-bisectrix, trigonal-binary and trigonal crystallographic planes. However the amount of computation involved in calculating cross sectional areas is rather excessive and the observed values of the de Haas van Alphen effect frequencies are not a convenient form of data to use in an extensive search of parameter space.

In this investigation another approach was adopted. The experimental calipers reported by Bogle, Coon, and Grenier (1969) were used to deduce the approximate locations of several points on the Fermi surface. The procedure was then to solve the pseudopotential secular equation and thus calculate the energy eigenvalues at these points. In principle there should be a common eigenvalue, points situated on the first zone hole surface should have their lowest energy levels equal, while points located on the second zone lenses should have theix second energy levels equal to the same value. If the points are designated $\mathrm{P}_{1}, \mathrm{P}_{2}, \ldots, \mathrm{P}_{\mathrm{N}}$ and the corxesponding energy levels are written $E_{1}, E_{2}, \ldots, E_{N}$. The mean energy at all the points is equal to $\overline{\mathrm{E}}$, where,

$$
\vec{E}=\frac{1}{N} \sum_{i=1}^{N} E_{i}
$$

A criterion of good fit was defined by introducing the quantity $\Delta \mathrm{E}$, where 


$$
\Delta E=\left[\frac{1}{N} \sum_{i=1}^{N}\left(E_{i}-\bar{E}\right)^{2}\right]^{\left(\frac{1}{2}\right)}
$$

Using this method a search was carried out in parameter space until those regions were found for which $\Delta \mathrm{E}$ was as small as possible.

Once a pseudopotential had been found for which $\Delta \mathrm{E}$ was small, the value of $\overline{\mathrm{E}}$ was regarded as a provisional approximation to the Fermi energy. The next step was to calculate the energy bands and to check whether the resulting band structure would generate a Fermi surface having the correct topology. The most delicate and sensitive feature of the Fermi surface of mercury is the $\beta$ arms. The Fermi energy was arbitrarily selected to make the minimum cross sectional areas of these arms come to the experimental value of 74 Tesla. With this value for the Fermi energy dif-ferent extremal cross sectional areas were then calculated and compared with experiment.

This method of defining the Fermi energy is obviously somewhat arbitrary. Mercury is a compensated metal and ideally the Fermi energy should be defined so as to satisfy this condition. However this involves a density of states calculation which requires much computation. The method that was used is approximate and the best test of its validity is the consistency with which the other calculated cross sectional areas agree with experiment. It is also important that the Fermi 
energy defined in this way should not differ greatly from the mean energy $\overline{\mathrm{E}}$ at the experimental points which were used in the original fitting procedure.

It is possible that some of these experimental points may have been slightly mislocated due to experimental error. Ideally the points should be located as accurately as possible and there should be a sufficient number of them so that if a few of them are slightly in error, the effect is minimized. It is also desirable to distribute the points so that all the important sections of the Fermi surface are represented. Using a given type of pseudopotential it may happen that it is not possible to achieve a very good fit and $\Delta E$ may never become very small no matter how the parameters are varied. If this happens it may be that some of the points are seriously mislocated, or it may be that the type of pseudopotential that is being used is inadequate in some way. It could be that a local pseudopotential is being used in a situation where this is not a good approximation and a non-local pseudopotential is needed. Another possibility is that spin-orbit coupling has been neglected when it should really be included. If there are good reasons for supposing that a certain type of pseudopotential must be a good approximation and poor fits are obtained then it is reasonable to suppose that some of the points are mislocated and better fits may be obtained if these points are shifted by a small amount. This method of correction was 
not used in the research described in this thesis, this was because of the uncertainty in the pseudopotential itself. It was not clear in advance which type of pseudopotential would be best for mercury.

The experimental points that were used were located on the second zone lenses and on various parts of the first zone hole surface. Among the epxerimental dimensions reported by Bogle, Coon, and Greniex (1969) were the radial. calipers of the Fermi surface,

$$
k_{\text {Lens }}^{L-T}, k_{\text {Lens }}^{L-U}, k_{\text {in }}^{L-U} k_{\text {in }}^{X-U}, k_{\text {out }}^{L-U}, k_{\text {out }}^{T-U}, k_{\text {out }}^{X-U}, k_{\text {out }}^{T-W} .
$$

The meaning of this notation is that $k_{\alpha}^{A-B}$ refers to the distance in $k$ space from the point $A$ to the Fermi surface measured in the direction of the point $B$. The suffix $\alpha$ indicates the section of the Fermi surface referred to. If the word lens is used as a suffix it means that the surface referred to is the second zone lens. The suffixes 'in' and 'out' refer to internal and external calipers of the first zone hole surface. Table (IV.I) gives the experimental values of these calipers as reported by Bogle et al.

Table (IV.I)

Experimental calipers of the Fermi surface as determined by Bogle, Coon, and Grenier (units are in $\mathrm{A}_{0}^{-1}$ )
$\frac{k_{\text {Lens }}^{L-\Gamma}}{0.176}$
$\underline{k_{\text {Lens }}^{L-U}}$
$\mathrm{k}_{\text {in }}^{\mathrm{L}-\mathrm{U}}$
$\mathrm{k}_{\text {in }}^{\mathrm{X}-\mathrm{U}}$
$\mathrm{k}_{\text {out }}^{\mathrm{L}-\mathrm{U}}$
$\mathrm{k}^{\mathrm{T}-\mathrm{U}}$
$\mathrm{k}_{\text {out }}^{\mathrm{X}-\mathrm{U}} \quad \mathrm{k}_{\text {out }}^{\mathrm{T}-\mathrm{W}}$

$$
0.538 \quad 0.90
$$
$0.30 \quad 1.14$
0.77
0.765
0.90 
These 8 points on the Fermi surface were used to determine what will subsequently be called an 8 point fit. When this research was started it was hoped that achieving a good fit to these points would uniquely determine a good Fermi surface. It was later discovered that this was not a sufficient condition and that a pseudopotential that gave a good fit to these 8 points could generate a set of energy bands and a Fermi surface that was unsatisfactory in other ways.

Only two of the 8 points were located on the lenses while five were situated around the $\tau$ minimum cross section It seemed possible that the $\tau$ section of the Fermi surface was over-represented and i.t was decided to look for more experimental points on the lenses.

Figure (7) of the paper of Bogle, Coon, and Grenier (1969) shows a plot of the experimental data for the (1.īo) mirror plane cross section of the second zone lens. Six experimental points were taken from an enlarged version of this graph and used to supplement the previous 8 points. It was hoped that the increased errors which were probably inherent in the use of these other six points would be offset by having a more general sampling of the Fermi surface. Three of the six points were taken from the half of the lens that lay inside the first Brillouin zone, the other three were taken from the half that was outside the zone. It eventually became clear that achieving a good fit 
to either the 8 experimental points or to the 14 points would not necessarily give a Fermi surface of the correct topology or yield cross sectional areas that were in very good agreement with the accurate de Haas van Alphen effect measurements of Poulsen (1970), however the procedure of varying the pseudopotential parameters so as to obtain a good. 8 point fit or a good 14 point fit was effective as an efficient means of exploring parameter space to find regions of interest.

3. The Coordinates in Reciprocal Space of the Experimental Points

A convenient way to describe the locations of the experimental points used in the fitting procedure is in terms of the $X, Y, Z$ coordinate system shown in the diagram of the Brillouin zone which is illustrated in Figure (III.1). For the sake of comparison the coordinates of the important symmetry points on the Brillouin zone are first given in Table $(I V .2)$.

Table IV.2)

The coordinates in reciprocal space of important symmetry points on the Brillouin zone. (The dimensions are in reciprocal atomic units.)

$\begin{array}{cccc}\text { Point } & \frac{\mathrm{KX}}{\mathrm{KY}} & \underline{\mathrm{KZ}} \\ \Gamma & 0 & \underline{0} & 0 \\ \mathrm{~T} & 0 & 0 & 0.7485 \\ \mathrm{X}_{1} & 0.2776 & 0.4808 & 0.4990\end{array}$




\begin{tabular}{|c|c|c|c|}
\hline Point & $\underline{K X}$ & $\mathrm{KY}$ & $\underline{\mathrm{KZ}}$ \\
\hline L1 & 0.5552 & 0 & 0.2495 \\
\hline UI & 0.3310 & 0 & 0.7485 \\
\hline WI. & 0.3310 & 0.1911 & 0.7485 \\
\hline $\mathrm{U} 2$ & 0.1655 & 0.2866 & 0.7485 \\
\hline W3 & 0.5552 & 0.5795 & 0.2495 \\
\hline U3 & 0.3897 & 0.6750 & 0.2495 \\
\hline $\mathrm{S}$ & 0.3337 & 0.5779 & 0 \\
\hline$K^{\prime}$ & 0.6673 & 0.3853 & 0 \\
\hline$s^{\prime}$ & 0.6673 & 0 & 0 \\
\hline $\mathrm{K}$ & 0.4431 & 0.3853 & 0.4990 \\
\hline
\end{tabular}

The coordinates of the points used in the fitting procedure are listed in Table (IV.3).

\section{Table (IV.3)}

The coordinates in reciprocal space of the points on the Fermi surface that were used in the fitting procedure. (The dimensions are in reciprocal atomic units).

\begin{tabular}{|c|c|c|c|}
\hline Point & $\underline{\mathrm{KX}}$ & $\underline{\mathrm{KY}}$ & $\underline{\mathrm{KZ}}$ \\
\hline $\begin{array}{l}\mathrm{L}-\Gamma \\
\text { Lens }\end{array}$ & 0.4703 & 0 & 0.2113 \\
\hline $\mathrm{k}_{\text {Lens }}^{\mathrm{L}-\mathrm{U}}$ & 0.4385 & 0. & 0.5092 \\
\hline $\mathrm{k}_{\text {in }}^{\mathrm{L}-\mathrm{U}}$ & 0.3600 & 0 & 0.6839 \\
\hline$k_{\text {out }}^{I-U}$ & 0.3080 & 0 & 0.7997 \\
\hline $\mathrm{k}_{\text {in }}^{\mathrm{X}-\mathrm{U}}$ & 0.2246 & 0.3889 & 0.6170 \\
\hline${ }_{k} \begin{array}{l}X-U \\
\text { out }\end{array}$ & 0.1423 & 0.2465 & 0.8000 \\
\hline
\end{tabular}




\begin{tabular}{|c|c|c|c|}
\hline Point & $\mathrm{KX}$ & $\mathrm{KY}$ & $\mathrm{KZ}$ \\
\hline $\mathrm{k}_{\text {out }}^{\mathrm{T}-\mathrm{U}}$ & 0.4075 & 0 & 0.7485 \\
\hline $\mathrm{k}_{\text {out }}^{\mathrm{T}-\mathrm{W}}$ & 0.4124 & 0.2381 & 0.7485 \\
\hline $\begin{array}{l}3 \text { points on } \\
\text { second zone }\end{array}$ & $\begin{array}{l}\text { the trigor } \\
\text { lens. (Ins }\end{array}$ & $\begin{array}{l}\text { Ctrix } \\
\text { Brillo }\end{array}$ & $\begin{array}{l}\text { tion of the } \\
\text { e.) }\end{array}$ \\
\hline Point & $\mathrm{KX}$ & $\underline{K Y}$ & $\mathrm{KZ}$ \\
\hline LI & 0.4035 & 0 & 0.4015 \\
\hline L2 & 0.4159 & 0 & 0.3415 \\
\hline 亡3 & 0.4298 & 0 & 0.2733 \\
\hline \multicolumn{4}{|c|}{$\begin{array}{l}3 \text { points on the trigonal-bisectrix cross section of the } \\
\text { second zone lens (External to the Brillouin zone.) }\end{array}$} \\
\hline Point & $\mathrm{KX}$ & $\underline{K Y}$ & $\mathrm{KZ}$ \\
\hline L4 & 0.5095 & 0 & $0.4847^{\circ}$ \\
\hline L5 & 0.5817 & 0 & 0.4109 \\
\hline L6 & 0.6219 & 0 & 0.3506 \\
\hline
\end{tabular}

\section{The Fermi Energy}

It often happens that when a band structure has been calculated using several different theoretical methods, there seems to be a surprisingly large variation in the predicted Fermi energies. In the case of mercury there have been several semi-empirical pseudopotential calculations reported while Keeton and Loucks (1966) have carried out a 
first principles calculation using the method of relativistic augmented plane waves.

The local pseudopotential calculations of Brandt and Rayne (1966), Dishman and Rayne (1968) which also included spin-orbit coupling, and of Bogle, Grenier, and Coon (1969), all produced Fermi energies that were close to the free electron energy of 0.5240 Rydbergs. In fact it is characteristic of weak local pseudopotentials that they give a Fermi energy close to the free electron value. By contrast Keeton and Loucks RAPW energy bands had the Fermi energy at the much higher level of 0.680 Rydbergs relative to the bottom of the conduction band. This variation of the Fermi energy also occurs in other instances.

In the case of zinc, Harrison (1962) calculated an energy band structure using a modified OPW technique that was equivalent to using a rather complicated non-local pseudopotential. The Fermi energy was reported as 0.708 Rydbergs which is equivalent to the free electron energy of 0.7076 Rydbergs. A non-relativistic APW calculation for zinc was reported by Mattheiss (1964). This gave a Fermi energy of 0.76 Rydbergs. The non-local pseudopotential of Stark and Falicov (1967) gave a Fermi energy of 0.8005 Rydbergs.

An extreme case of this type of variation occurs in tungsten. Loucks (1966) has reported a Fermi energy of 0.548 Rydbergs from an APW-calculation, but when an RAPW calcula- 
tion was performed the conduction band was much broader with a Fermi energy of 0.760 Rydbergs.

There do not appear to be any published experimental values for the Fermi enexgy of mercury, but it seems reasonable to suppose that an RAPW calculation should give a description of the energy bands of mercury that is closer to the truth than the local pseudopotential approximation. For this reason when the search in parameter space was carried out particular attention was paid to the question of the Fermi energy .

It was earlier mentioned that the parameters $\mathrm{v}(\mathrm{s})$ and $\mathrm{v}(\mathrm{p})$ of Stark and Falicov's pseudopotentials, equation (2.34), were both zero in the case of zinc, but there was a strong repulsive term from the $3 d$ level causing $v(d)$ to have the large value of 3.15 Rydbergs. A similar result was given for cadmium where $v(s)$ was again zero while $v(p)$ was comparatively small at 0.38 Rydbergs, the parameter $v(d)$ corresponding to the 4 d level was relatively large at 1.78 Rydbergs. The free electron Fermi energy of zinc is 0.708 Rydbergs while Stark and Falicov obtained 0.8005 Rydbergs. In the case of cadmium the free electron Fermi energy is equal to 0.557 Rydbergs, while stark and Falicov obtained a value of 0.651 Rydbergs. These elevated Fermi energies can be understood as an effect of the non-local pseudopotential operations. The d-like projection operator has the effect of 
raising the energy of all wave-functions which contain a significant d-like component, similarly the p-like projection operator will raise the energy levels of all states with a $p$-like component. The bottom of the bands around $\Gamma$ are s-like this means they will be unaffected by the $\mathrm{p}$ and d-like non-local operators. This means that if s-like projection operators are excluded from the pseudopotential the Fermi energy will be increased, because the energy levels away from $\Gamma$ and towards the zone boundaries will be varied in energy while the bottom of the bands remain fixed. If an $s-l i k e$ projection operator is included the Fermi energy is reduced because in this case the bottom of the bands will be raised more in energy than the remainder. These arguments are dependent upon the parameters $v(s), v(p)$, and $v(d)$ all being positive.

5. The Choice of a Psevidopotential

In Chapter II of this thesis (section 6) the question of the choice of a suitable approximation for the pseudopotential was discussed. In view of the fact that mercury lies in the same column of the periodic table as zinc and cadmium and like them has two electrons in the conduction band it was decided to undertake the investigation into the Fermi surface of mercury using an analogous pseudopotential to that used by stark and Falicov for these two metals. The 
pseudopotentials used in most of this research were of the form

$$
H_{p}=T+W+R 5 S \mid 5 s>\langle 5 s|+R 5 P| 5 p><5 p|+R 5 D| 5 d><5 d|
$$

Stark and Falicov included spin-orbit coupling in their calculations. In the case of mercury there are no specific features of the Fermi surface which owe their existence to the spinorbit effect. For this reason spin-orbit coupling was not included in most of this research. However some investigations were made into the order of magnitude of this interaction on the band structure.

It was not clear a priori what values of the parameters R5S, R5P, and R5D would give the best pseüdopotential. It was assumed that a region of interest might lie in that part of parameter space where $\mathrm{R} 5 \mathrm{~S}, \mathrm{R} 5 \mathrm{P}$, and $\mathrm{R} 5 \mathrm{D}$ had the values corresponding to the OPW-pseudopotential of equation (2.7) in which,

$$
R 5 S=\left(E_{k}-E_{5 s}\right), R 5 P=\left(E_{k}-E_{5 p}\right), R 5 D=\left(E_{k}-E_{5 d}\right) .
$$

In this approximation $E_{k}$ could be taken to be the energy of the $6 \mathrm{~s}$ atomic levels as computed by Herman and skillman, while $E_{5 s}, E_{5 p}$, and $E_{5 d}$ would be the corresponding energies of the $5 \mathrm{~s}, 5 \mathrm{p}$, and $5 \mathrm{~d}$ atomic energy levels. The energies in the solid will be different from the situation in the free atom, however it is reasonable to suppose that the inner core states will not be strongly perturbed by the crystal field. On the 
other hand the 5s, 5p, and $5 \mathrm{~d}$ levels might be strongly perturbed, so the use of Herman and Skillmans atomic energy levels may not be a good guide to the values of the non-local parameters.

In Stark and Falicov's calculations the d-like operators were the dominant terms in the non-local pseudopotential. This could be due to a hybridization effect due to the proximith of the d bands to the conduction band. In the case of zinc Mattheiss (1964) has calculated that in this metal the 3d band was about 0.5 Rydbergs below the bottom of the $(4 s-4 p)$ bands. It was mentioned earlier that there is some evidence that the $5 d$ bands actually cut across the bottom of the $6 s-6 p$ conduction band in mercury, so hybridization effects could be important in mexcury too.

Using a pesudopotential of the form given in equation (4.3) an extensive search was carried out in parameter space to look for regions of interest.

6. Methods of Calculating Pseudopotential Band Structures and Fermi Surfaces for Mercury

A variety of computer programs were developed to calculate the energy bands of mercury using non-local pseudopotentials. Some of these programs were used in an exploration of parameter space to find regions of good fit to the experimental Fermi surface. Another set of programs was built which could calculate the cross sectional areas and the cyclotron 
masses of plane sections of the Fermi surface generated by a given pseudopotential. A program was also developed that could calculate the energy bands for a non-local pseudopotential with spin-orbit coupling included. All of these programs were based on a similar principle. The simplest to explain initially are those that calculated the band structure for a non-local pesudopotential omitting spin-orbit coupling.

The parametrized pseudohamiltonian whose energy eigenvalues it was desired to calculate was of the form given in equation $(4.3)$

$$
H_{p}=T+W+R 5 S \mid 5 s>\langle 5 s|+R 5 p| 5 p><5 p|+R 5 D| 5 d><5 d|
$$

where $|5 s\rangle,|5 p\rangle$, and $|5 d\rangle$ are tight binding linear combinations of atomic $5 \mathrm{~s}, 5 \mathrm{p}$, and $5 \mathrm{~d}$ wavefunctions as calculated by Herman and Skillman.

In analogy to equation (2.23) of Chapter 1 the general matrix element to be calculated is of the form

$$
\begin{aligned}
& <\underline{k}-G_{1}\left|H_{p}\right| \underline{k}-G_{2}>=\left|\underline{k}-G_{1}\right|^{2} \delta_{G_{I}} G_{2}+W\left(G_{2}-G_{1}\right) \\
& +\frac{4 \pi}{\Omega}\left[R 5 S P_{0}(\cos \alpha) I_{5 s}\left(\left|\underline{k}_{-} G_{I}\right|\right) I_{5 s}\left(\left|k-G_{2}\right|\right)\right. \\
& +3 R 5 P_{1}(\cos \alpha) I_{5 p}\left(\left|k-G_{1}\right|\right) I_{5 p}\left(\left|\underline{k}-G_{2}\right|\right) \\
& \left.+5 R 5 D P_{2}(\cos \alpha) I_{5 d}\left(\left|k-G_{1}\right|\right) I_{5 d}\left(\left|k-G_{2}\right|\right)\right] .
\end{aligned}
$$

where $\alpha$ is the angle between the vectors $\left(\underline{k}_{-} \underline{G}_{1}\right)$, and $\left(\underline{k}-\underline{G}_{2}\right)$. $\mathrm{P}_{0}, \mathrm{P}_{1}$, and $\mathrm{P}_{2}$ are the Legendre polynomials for which, $\mathrm{P}_{0}(\cos \alpha)=1, \quad \mathrm{P}_{1}(\cos \alpha)=\cos \alpha, \mathrm{P}_{2}(\cos \alpha)=\frac{1}{2}\left(3 \cos ^{2} \alpha-1\right)$. 
The integrais $I_{5 s}, I_{5 p}$, and $I_{5 d}$ are defined by

$$
\begin{aligned}
& I_{5 s}(|k|)=\int j_{0}\{|k| \times r\} \times P_{5 s}(r) d r \\
& I_{5 p}(|k|)=\int j_{I}\{|k| \times r\} r P_{5 p}(r) d r \\
& I_{5 d}(|k|)=\int j_{2}\{|k| \times r\} r P_{5 d}(r) d r .
\end{aligned}
$$

where $P_{5 s}(r), P_{5 p}(r)$, and $P_{5 d}(r)$ are the normalized radial functions of Herman and skillman. In equation (4.4), $\Omega$ is the volume of the unit cell of the real lattice, and $W\left(G_{2}-G_{1}\right)$ is the local pseudopotential corresponding to the reciprocal vector $\left(\underline{G}_{2}-\underline{G}_{1}\right)$.

In all the calculations that have been performed reduced atomic units have been used throughout. Distances in reciprocal space were in reciprocal atomic units while all energies were expressed in Rydbergs.

To speed up the calculations the integrals $I_{5 s}(|k|)$ $I_{5 p}(|k|)$, and $I_{5 d}(|k|)$ were first calculated separately for a range of values of $|k|$, equally spaced between 0 and 5 at intervals of 0.01 reciprocal atomic units. The values of these integrals were punched on to cards which were incorporated as part of the data of every program. An interpolation subroutine was built into the programs so that the value of the integrals could be extracted when needed for any value of $|k|$ within the tabulated range. 
When the energy eigenvalues were to be calculated at a point $\underline{k}$ in reciprocal space the following procedure was followed in all the programs. The point $\underline{k}$ was first translated into the equivalent position in the first Billouin zone. It was then subjected to a series of inversions, reflections in mirror planes, and rotations by $\pm 120^{\circ}$ about the trigonal axis until $\underline{k}$ was in an equivalent position in the smallest reduced section of the Brillouin zone. THe diagram in Figure (III.I) shows the Brillouin zone of mercury with the smallest reduced section shown outlined. In the figure the origin of a rectangular coordinate system is at $\Gamma$ with the $\mathrm{z}$ axis positive in the direction IT. The $\mathrm{X}$ axis is perpendicular to TT lying in the mirror plane passing through the points $T, U_{1}$, and $I_{1}$. $L_{1}$ is at the centre of a pseudohexagonal face and the $x$ axis cuts this face at $s^{\prime}$. The $x$ axis is measured positive in the direction $\Gamma S^{\prime}$. The $\mathrm{Y}$ axis is perpendicular to the $(\mathrm{X}-\mathrm{Z})$ plane, with its positive direction to the right in the figure. Before building the band structure programs another program was built that could calculate the locations of the points of the reciprocal lattice and then arrange them in shells of increasing distance from an arbitrary point in $k$ space. This program was applied to all the principal points shown in Figure (III.I) which are situated on the boundary of the smallest reduced section. The results of these calculations were used to determine the appropriate order of the 
secular equation at these points. For example the $\Gamma$ point at the origin has itself as its nearest neighbour, there are 6 second nearest neighbours, 6 third nearest neighbours, and 2 fourth nearest neighbour reciprocal vectors and so on. This means that the appropriate order of secular equation at $\Gamma$ should be $(1 \times 1),(7 \times 7),(13 \times 13),(15 \times 15)$ etc. Any other numbers would deviate from the symmetry requirements and cause a distortion in the calculated energy bands. The order of secular equation used at the principal points varied. For most local pseudopotential calculations it was adequate to have the following orders at the various points i $\Gamma(15), T(14), X(20), L(12), U(17), W(14), S(14)$, and $\mathrm{K}(16)$. When non-local pseudopotentials were used it was found that the convergence was not always very good when the secular equations were this small. Accordingly for most non-local pseudopotential calculations the orders used were; $F(33), T(40), X(34), L(34), U(38), W(40), S(34)$, and $K(40)$. When calculating the energy bands at a given point $\underline{k}$ in reciprocal space the procedure was as follows. The program first translated $k$ to an equivalent position in the first Brillouin zone and in the smallest reduced section. The next step was to search for the nearest principal point to the new position of $\mathrm{k}$. The matrix elements $\left\langle\underline{k}_{-}-G_{1}\left|H_{p}\right| \underline{k}-G_{2}\right\rangle$ were then calculated, where the vectors $G_{1}, G_{2}$, etc. were selected from the symmetrical set of vectors belonging to 
this principal point. The formula for these matrix elements was given in equation (4.4). In calculating such an element involving two reciprocal vectors $G_{1}$ and $G_{2}$ which may be the same, the program first calculated the cartesian coordinate of the vectors $\left(\underline{k}-\underline{G}_{1}\right)$ and $\left(\underline{k}-G_{2}\right)$. It then calculated the moduli of these two vectors and the cosine of the angle between them. The moduli were used to find the values of the functions $I_{5 s}, I_{5 p}$, and $I_{5 d}$. The cosine of the angle between the vectors was used to calculate the values of the Legendre polynomials. The local pseudopotential $\mathrm{W}\left(\mathrm{G}_{2}-\underline{G}_{1}\right)$ was calculated using a special subroutine called ADDRES.

Once all the matrix elements were calculated the program diagonalized the matrix and arranged the resulting energy eigenvalues in ascending order. 7. Programs to Calculate Cross Sectional Areas of the Fermi

A given pseudopotential will generate a set of constant energy surfaces in $\underline{k}$ space. One of these surfaces will be the theoretical 'Fermi surface' appropriate to the particular pseudopotential. It will not be known initially what the Fermi energy will be, but if it is guessed or arbitrarily selected in some way then, it is possible to build programs which search for the required energy contour and follow it around in a plane section. In this way cross sectional areas of the 'Fermi 
surface' can be calculated and compared with the observed de Haas van Alphen effect periods.

A set of programs was built which calculated such cross sectional areas and simultaneously found the 'rigid band value' of the cyclotron mass of the orbit. In a typical calculation a point $\mathrm{O}^{\prime}\left(\underline{\mathrm{k}}_{1}, \underline{\mathrm{k}}_{2}, \underline{\mathrm{k}}_{3}\right)$ was selected and a plane through $O^{\prime}$ perpendicular to the magnetic field $\underline{H}$ intersected the 'Fermi surface' in a closed curve whose area was to be calculated.

It was convenient to use two coordinate systems in these calculations, the two systems being related through a set of Euler angles. The $X, Y$, and $Z$ axes used to define positions in the Brillouin zone have been described. Unit vectors $I, I, \underline{K}$ can be drawn through $O^{\prime}$ parallel to these axes. The other set of mutually orthogonal axes $x, y$, and $z$ were oriented so that the positive z direction was parallel to the magnetic field $\underline{H}$. Unit vectors $\underline{i}, j, \underline{k}$ can also be drawn through $O^{\prime}$ parallel to these other axes. The relationship between the two sets of axes can be seen by considering the following sequence of operations. The explanation is clearer if reference is made to Figure (IV.I) which shows the Euler angles.

Beginning with the orthogonal vectors $I, \underline{J}$, and $\underline{K}$, (i) Rotate by an angle $\phi$ around $\underline{K}$, this gives a new set of axes and unit vectors $I^{\prime}, J^{\prime},\left(K^{\prime}=K\right)$. 


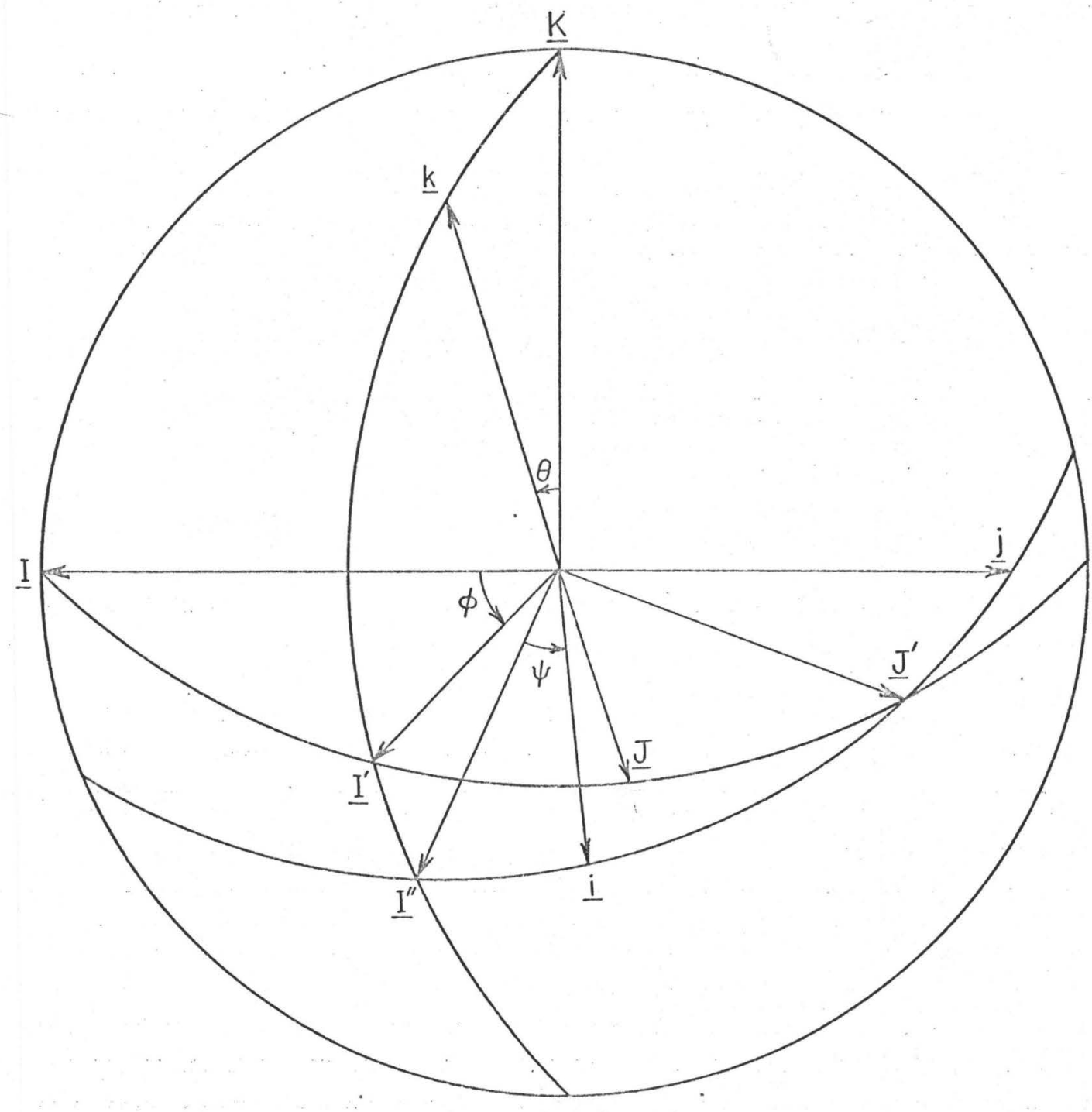

Figure (IV.I)

The system of Euler angles used in the programs that calculated cross sectional areas of the Fermi surface. 
(ii) Rotate by an angle $\theta$ around the new ' $y$ ' axis 'the line of nodes' to get a new set of axes I", J', $\underline{k}$.

(iii) Rotate by an angle $\psi$ around the new $z$ axis to get the final set of axes $\underline{i}, \underline{j}, \underline{k}$.

Table (IV.4) represents the direction cosines of the unit vectors $(\underline{i}, \underline{j}, \underline{k})$ relative to the original set of unit vectors $(I, \underline{I}, \underline{K})$.

\section{Table (IV.4).}

The table gives the mutual scalar products between the two sets of unit vectors $(\underline{i}, \underline{j}, \underline{k})$ and $(I, \underline{J}, \underline{K})$, in terms of the Euler angles $(\theta, \phi, \psi)$.

\begin{tabular}{|l|c|c|}
\multicolumn{1}{c}{$\underline{\mathrm{I}}$} & \multicolumn{2}{c}{$\underline{\mathrm{J}}$} \\
\cline { 2 - 4 } $\cos \theta \cos \phi \cos \psi$ & $\cos \theta \sin \phi \cos \psi$ & $-\sin \theta \cos \psi$ \\
$-\sin \phi \sin \psi$ & $+\cos \phi \sin \psi$ & \\
\hline$-\cos \theta \cos \phi \sin \psi$ & $-\cos \theta \sin \phi \sin \psi$ & $\sin \theta \sin \psi$ \\
$-\sin \phi \cos \psi$ & $+\cos \phi \cos \psi$ & \\
\hline $\sin \theta \cos \phi$ & $\sin \theta \sin \phi$ & $\cos \theta$ \\
\hline
\end{tabular}

The set of Euler angles $(\theta, \phi, \psi)$ thus described the direction of $\underline{H}(\theta, \phi)$ in terms of spherical polar angles. The angle $\psi$ gave the direction of $\underline{i}$ in the plane perpendicular to $\underline{H}$.

The cross sectional areas of greatest interest were the extremal areas which could be compared with the de Haas van Alphen periods. When these areas were calculated the origin $0^{\prime}\left(k_{1}, k_{2}, k_{3}\right)$ was previously selected so that it was 
situated in a position somewhere near the centre of the closed contour. The Euler angles $\theta$ and $\phi$ were given by the direction of $\underline{H}$. The angle $\psi$ was initially set equal to zero and the program carried out an exploration along the $\mathrm{x}$ axis until. the Fermi energy contour was located. $\psi$ was then incremented in equal steps around a complete circle. As $\psi$ was increased the $\mathrm{x}$ axis was rotated, and with each increment in $\psi$ the program made a search along this axis until it had found the energy contour. The area of the contour was then obtained using an integration subroutine. The area is given by

$$
A=\oint \frac{1}{2} r^{2} d \psi
$$

The values of $r\left(\psi_{i}\right)$ having been found for equally spaced intervals of $\psi_{i}$, the integration subroutine was then used to compute the resulting area. The subroutine made use of a combination of Simpson's rule and Newton's $\frac{3}{8}$ rule. In most of the calculations $\psi$ was incremented at intervals of 5 degrees, although some calculations used an interval of 10 degrees.

As extremal cross sectional areas were of interest it was important to locate $O^{\prime}$ as near as possible to the correct position to obtain extremal areas. In the case of the second zone lens, $O^{\prime}$ was put at the centre of the lens at $L$. When computing the extremal area of the $\beta$ arms, $O^{\prime}$ was located on the XK line near the centre of the minimum cross section. 
The programs that were used were limited to contours for which $r(\psi)$ was a single valued function of $\psi$. More general programs could have been constructed to compute the areas of more complicated closed contours, however these would have required a lot of computer time and such programs were not used.

The formula for the cyclotron mass of the orbit is given by

$$
m_{C}^{*}=m\left\{\frac{l}{\pi} \frac{\partial A}{\partial \varepsilon}\right\}
$$

where it is assumed that $A$ is in (atomic units) ${ }^{-2}$ and $\varepsilon$ is in Rydbergs, $m$ is the free electron mass.

The cross sectional area is given by equation (4.5)

$$
A=\oint \frac{1}{2} r^{2} d \psi
$$

so that

$$
\begin{aligned}
& \frac{\partial A}{\partial \varepsilon}=\frac{\partial}{\partial \varepsilon} \int\left(\frac{1}{2} r^{2}\right) d \psi \\
& \frac{\partial A}{\partial \varepsilon}=\oint r\left(\frac{\partial r}{\partial \varepsilon}\right) d \psi
\end{aligned}
$$

giving

$$
\frac{m_{c}^{*}}{m}=\frac{1}{\pi} \int r\left(\frac{\partial r}{\partial \varepsilon}\right) d \psi
$$

The programs simultaneously calculated the reduced effective mass of the orbits in the following way. For each value of $\psi$, the radial'distance $r(\psi)$ out to the Fermi energy contour was determined by the search procedure that has just been described. The program then calculated the energy at 
the slightly larger radius $(r+\delta r)$. In this way the derivative $\left(\frac{\partial r}{\partial \varepsilon}\right)_{\psi}$ was determined. The product of $r(\psi)$ and of $\left(\frac{\partial r}{\partial \varepsilon}\right)_{\psi}$ was found for each incremented value of $\psi$ and the cyclotron mass was then found using the integration subroutine and making use of equation (4.7).

Whenever the program was to calculate the energy eigenvalues at a point $P$ which was at a position $r i$ relative to $\mathrm{O}^{\prime}$, the coordinates of the point were first converted into the $X, Y, Z$ system used to locate points in the Brillouin zone. If the point $\mathrm{O}^{\prime}$ was at position vector $\mathrm{O}^{\prime}$, then $?$ was at the vector position

$$
P=\underline{o}^{\prime}+r \underline{i}
$$

Expressed in terms of the unit vectors I, J, $\underline{K}$

$$
\underline{O}^{\prime}=k_{1} I+k_{2} I+k_{3} \text {. }
$$

and

$$
\underline{i}=(\underline{i} \cdot I) \underline{I}+(\underline{i} \cdot J) \underline{J}+(\underline{i} \cdot \underline{K}) \underline{K} .
$$

This means that the $X, Y, Z$ coordinates of $P$ were given by

$$
\begin{aligned}
& \mathrm{P}_{\mathrm{X}}=\mathrm{k}_{1}+(\cos \theta \cos \phi \cos \psi-\sin \phi \sin \psi) r \\
& \mathrm{P}_{Y}=\mathrm{k}_{2}+(\cos \theta \sin \phi \cos \psi+\cos \phi \sin \psi) r \\
& \mathrm{P}_{Z}=\mathrm{k}_{3}-\sin \theta \cos \psi r .
\end{aligned}
$$

In deriving these coordinates the scalar products listed in Table (IV.4) have beer used.

Once the position of P was located in terms of the conventional system of coordinates, $P$ was then subjected to 
the process of translation into the Brillouin zone) and subjected to the necessary symmetry operations to transport it to an equivalent position inside the smallest reduced section. The nearest principal point was then selected and the corresponding secular matrix was set up and diagonalized. 


\section{CHAPTER V \\ RESULTS OF THE PSEUDOPOTENTIAL CALCULATIONS}

1. Some Examples of 8 Point Fits

In Table (V.I) the 8 point figure of merit is compared for several local pseudopotentials. In each case the mean energy $\bar{E}$ was obtained from the appropriate energy eigenvalues at the 8 experimental points. A convenient figure of merit was given by the number $\Delta E_{*}$ where

$$
\Delta \mathrm{E}=\left[\sum_{i=1}^{i=8}\left(E_{i}-\bar{E}\right)^{2}\right]^{\left(\frac{1}{2}\right)} \times 10^{3} .
$$

The pseudopotentials given in the Table are (i) the free electron model, (ii) the local pseudopotential of Heine and Animalu, (iii) the local pseudopotential of Brandt and Rayne, and (iv) the best two parameter local pseudopotential.

The best two parameter local pseudopotential was a model in which $W_{L}$ and $W_{X T}\left(W_{X T}=W_{X}=W_{T}\right)$ were the only non zero parameters and which gave a better fit using the 8 point criterion than any other similar two parameter model. In these calculations the order of the secular equations varied between $(12 \times 12)$ at $\mathrm{L}$ and $(20 \times 20)$ at $\mathrm{X}$. 
TABLE $(V . I)$

Examples of 8 point Fits [Energies in Rydbergs]

(i) The free electron model

\begin{tabular}{|c|c|c|c|}
\hline$k_{\text {Lens }}^{L-\Gamma}$ & $k_{\text {Lens }}^{L-U}$ & $k_{\text {in }}^{L-U}$ & $k_{\text {out }}^{I-U}$ \\
\hline 0.4926 & 0.4515 & 0.5973 & 0.5809 \\
\hline $\begin{array}{l}\mathrm{X}-\mathrm{U} \\
\mathrm{in}\end{array}$ & $\mathrm{k}_{\mathrm{out}}^{\mathrm{X}-\mathrm{U}}$ & $\mathrm{k}_{\text {out }}^{\mathrm{T}-\mathrm{U}}$ & $\mathrm{k}_{\text {out }}^{\mathrm{T}-\mathrm{W}}$ \\
\hline 0.5824 & 0.5666 & 0.5564 & 0.6061 \\
\hline
\end{tabular}

Mean energy $=0.5542$ Rydbergs. Figure of merit $=143$

(ii) The Heine-Animalu pseudopotential

\begin{tabular}{|c|c|c|c|}
\hline$k_{\text {Lens }}^{L-\Gamma}$ & $k_{\text {LenS }}^{\mathrm{L}-\mathrm{U}}$ & $\mathrm{k}_{\text {in }}^{\mathrm{L}-\mathrm{U}}$ & $\mathrm{k}_{\text {out }}^{\mathrm{L}-\mathrm{U}}$ \\
\hline 0.4861 & 0.4617 & 0.5327 & 0.5274 \\
\hline $\mathrm{k}_{\text {in }}^{\mathrm{X}-\mathrm{U}}$ & $\mathrm{k}_{\text {out }}^{\mathrm{X}-\mathrm{U}}$ & $k_{\text {out }}^{T-U}$ & $k_{\text {out }}^{T-W}$ \\
\hline
\end{tabular}

Mean energy $=0.5149$ Rydbergs. Figure of merit $=81$

(iii) The Brandt-Rayne Pseudopotential. $W_{1}=-0.066$ Ryd. $\underline{W}_{\mathrm{XT}}=0.047$ Ryd.

\begin{tabular}{|c|c|c|c|}
\hline$k_{\text {Lens }}^{L-\Gamma}$ & $\mathrm{k}_{\text {Lens }}^{\mathrm{L}-\mathrm{U}}$ & $k_{\text {in }}^{L-U}$ & $k_{\text {out }}^{L-U}$ \\
\hline 0.5096 & 0.5080 & 0.5292 & 0.5428 \\
\hline $\begin{array}{c}X-U \\
\text { in }\end{array}$ & $\begin{array}{l}\mathrm{X}-\mathrm{U} \\
\mathrm{k} \text { out }\end{array}$ & $k_{\text {out }}^{T-U}$ & $\begin{array}{l}\mathrm{T}-\mathrm{W} \\
\text { out }\end{array}$ \\
\hline 0.5303 & 0.5283 & 0.5277 & 0.5521 \\
\hline
\end{tabular}

Mean energy $=0.5285$ Rydbergs. Figure of merit $=39$. 
TABLE (V.1) (continued)

(iv) The best two parameter local pseudopotential $\underline{\mathrm{W}}_{\mathrm{L}}=-0.0879 \mathrm{Ryd} . \mathrm{W}_{\mathrm{XT}}=0.049 \mathrm{Ryd}$.

\begin{tabular}{|c|c|c|c|}
\hline$k_{\text {Lens }}^{L-\Gamma}$ & $k_{\text {Lens }}^{L-U}$ & $k_{\text {in }}^{\mathrm{I}-\mathrm{U}}$ & $\mathrm{k}_{\text {out }}^{\mathrm{I}_{-}-\mathrm{U}}$ \\
\hline 0.5207 & 0.5260 & 0.5139 & 0.5346 \\
\hline $\mathrm{k}_{\text {in }}^{\mathrm{X}-\mathrm{U}}$ & $\begin{array}{l}X-U \\
\text { out }\end{array}$ & $\mathrm{k}_{\mathrm{out}}^{\mathrm{T}-\mathrm{U}}$ & $\begin{array}{l}\mathrm{T}-\mathrm{W} \\
\mathrm{out} \\
\end{array}$ \\
\hline 0.5272 & 0.5177 & 0.5178 & 0.5355 \\
\hline
\end{tabular}

Mean energy $=0.5242$ Rydbergs. Figure of merit $=21$

2. Some Examples of 14 point Fits

The two examples of 14 point fits which are illustrated in Table (V.2) both belong to the form of non-local. pesudopotential given in equation (4.3). In each case the local pseudopotential parameters were limited to the two non-zero terms $\mathrm{W}_{\mathrm{L}}$ and $\mathrm{W}_{\mathrm{XT}}$. In these calculations the order of the secular equation varied between 33 at $\Gamma$, and 40 at $\mathrm{T}, \mathrm{W}$, and $\mathrm{K}$.

In this case the figure of merit was given by



The factor $\sqrt{\frac{8}{14}}$ was put. in to make $\Delta \mathrm{E}$ come to numbers of a comparable size as obtained when only 8 points were used. 
TABLE (V.2)

Examples of 14 point Fits (Energies in Rydbergs)

(i) Pseudopotential

\begin{tabular}{|c|c|c|c|}
\hline$k_{\text {Lens }}^{L-\Gamma}$ & $k_{\text {Lens }}^{L-U}$ & $\mathrm{k}_{\text {in }}^{\mathrm{L}-\mathrm{U}}$ & $\mathrm{k}_{\text {out }}^{\mathrm{L}-\mathrm{U}}$ \\
\hline 0.6448 & 0.6482 & 0.6545 & 0.6738 \\
\hline $\mathrm{k}_{\text {in }}^{\mathrm{X}-\mathrm{U}}$ & $\begin{array}{l}\mathrm{X}-\mathrm{U} \\
\text { out }\end{array}$ & $\begin{array}{l}\mathrm{k}^{\mathrm{T}-\mathrm{U}} \\
\text { out }\end{array}$ & $\begin{array}{l}\mathrm{T}-\mathrm{W} \\
\text { out }\end{array}$ \\
\hline 0.6546 & 0.6595 & 0.6556 & 0.6574 \\
\hline $\mathrm{I}_{1}$ & $\mathrm{~L}_{2}$ & $\mathrm{I}_{3}$ & $\mathrm{I}_{4}$ \\
\hline 0.6716 & 0.6659 & 0.6712 & 0.6512 \\
\hline $\mathrm{L}_{5}$ & $\mathrm{I}_{6}$ & & \\
\hline 0.6606 & 0.6632 & & \\
\hline
\end{tabular}

Mean energy $=0.6594$ Rydbergs, Figure of merit $=24$

(ii) Pseudopotential
$R 5 S=0, R 5 P=0, R 5 D=0$
$W_{L}=-0.0825, W_{X T}=0.0475$
$k_{\text {Lens }}^{\text {L-T }}$
$k_{\text {Lens }}^{\text {L-U }}$
$\mathrm{k}_{\text {in }}^{\mathrm{L}-\mathrm{U}}$
$k_{\text {out }}^{\text {L. U }}$
0.5133
0.5186
0.5140
0.5353
$\mathrm{k}_{\text {in }}^{\mathrm{X}-\mathrm{U}}$
$\mathrm{k}_{\text {out }}^{\mathrm{X}-\mathrm{U}}$
$\mathrm{k}_{\text {out }}^{\mathrm{T}-\mathrm{U}}$
$\mathrm{k}_{\text {out }}^{\mathrm{T}-\mathrm{W}}$
0.5282
0.5172
0.5170
0.5328
$\underline{\mathrm{L}_{1}}$
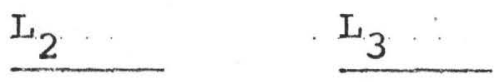
$\mathrm{I}_{4}$
0.5317
0.5277
0.5325
0.5225
$\underline{\mathrm{I}_{5}}$
$\mathrm{I}_{6}$
0.5273
0.5283

Mean energy $=0.5247$ Rydbergs. Figure of merit $=20.5$. 
3. The Best Two Parameter Model ( 8 Point Fit)

The two parametex local pseudopotential which gave the best fit to the 8 points, was given by $W_{L}=-0.0879$ Rydbergs, $W_{X T}=0.049$ Rydbergs. The corresponding band structure is shown in Figures (V.I) and (V.2). As is to be expected there is a strong resemblance between these energy bands and those generated by the Brandt-Rayne pseudopotential which was illustrated in Figures (III.4) and (III.5). The mean energy at the 8 points was 0.5242 Rydbergs, but this value for the Fermi energy gave $\beta$ arms that were too small. The following table shows how the calculated values of the minimum cross sectional area of the $\beta$ arms, and the corresponding cyclotron masses, varied as a function of 'Fermi energy'. The frequencies are in Tesla and the cyclotron masses are in units of $\mathrm{m}_{\mathrm{o}}$ (the free electron mass).

Fermi energy

0.5100

0.5150

0.5180

0.5185

0.5242

Cross Section (Tesla)

169

103

79.5

74.0

30.0 $\frac{\text { Cyclotron mass }}{\text { (units of } m_{0} \text { ) }}$

$-0.152$

$-0.099$

$-0.085$

$-0.084$

-0.050 .

The Fermi energy was arbitrarily set at 0.5185 Rydbergs so as to make the $\beta$ arms the correct size. 


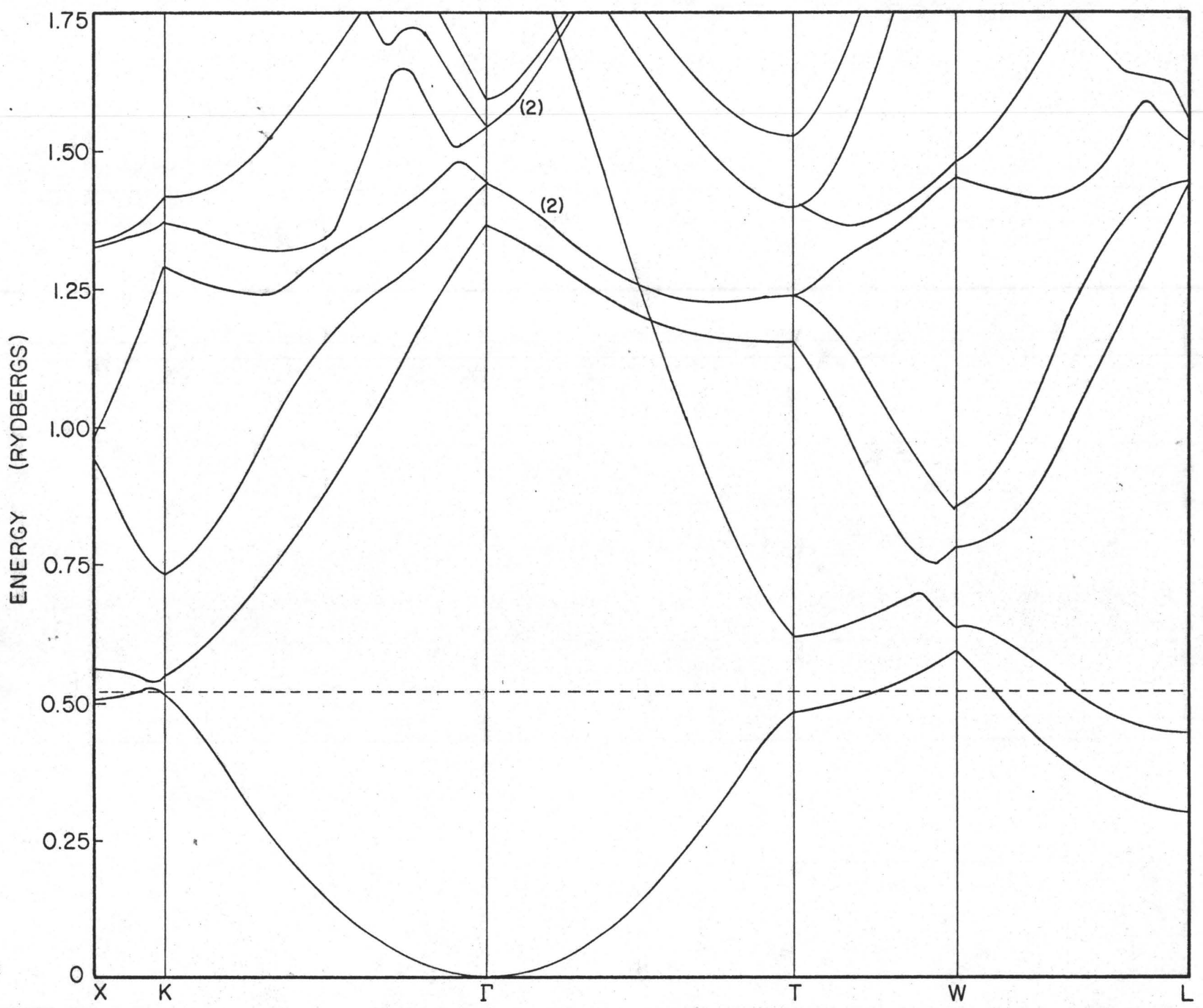
Figure (V.1) Energy bands for the 'best 8 point local pseudopotential', $\mathrm{W}_{L}=-0.0879$,
$\mathrm{W}_{\mathrm{XT}}=0.049$ Rydbergs. 


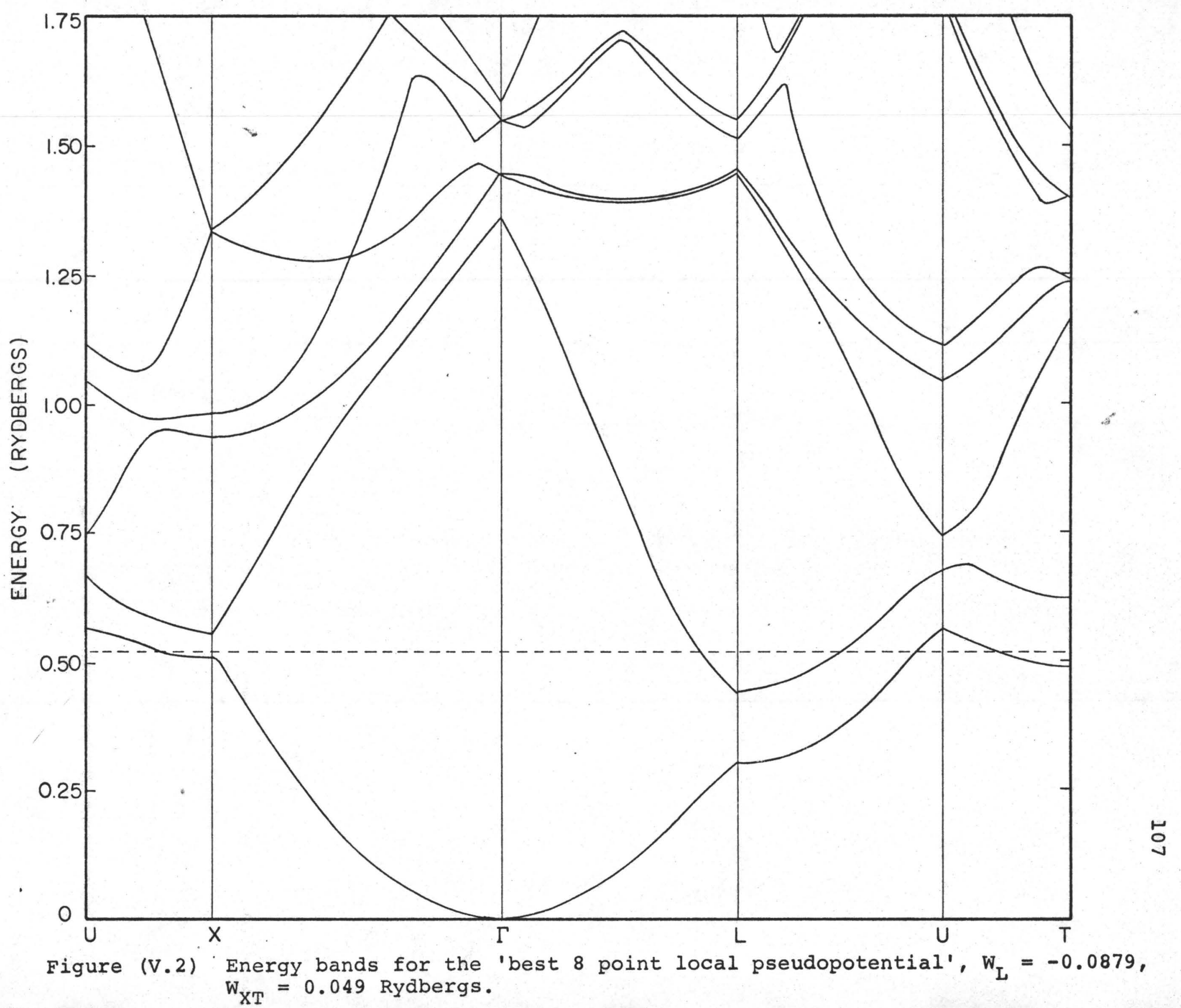


Figure (V.3) shows the variation of the calculated $\beta$ arm periods when the magnetic field is rotated in the trigonal-bisectrix plane. The experimental points of Poulsen (1970) are also shown superimposed on the graph. Figure (V.4) shows the corresponding set of calculated cyclotron masses. The two experimental points for comparison are taken from Brandt and Rayne (1966). These experimental masses were obtained from measurements of the variation of the amplitude of the de Haas-van Alphen oscillations as a function of temperature. The fact that the experimental masses are always larger than the calculated values is related to the electron-electron and electron-phonon mass enhancernent.

The corresponding graphs for the trigonal-bisectrix, trigonal-binary, and trigonal cross sections of the second zone lenses are shown in. Figures $(V .5), \ldots,(V .10)$. In each case the experimental values obtained by Poulsen are also shown. Cyclotron masses have not been measured for these exact orientations but Poulsen (1970) has given a ten parameter model for the variation of the cyclotron mass as a function of magnetic field orientation relative to the lenses. These parameters were derived from a fit to the cyclotron masses measured in other planes. The curves shown as experimental cyclotron masses in Figures (V.6), (V.8), and (V.10) were derived from this formula. 
Figures (V.3), .. , (V.10) show the calculated results for the 'best 8 point local pseudopotential' for which $\mathrm{W}_{\mathrm{L}}=-0.0879, \mathrm{~W}_{\mathrm{XT}}=0.049$ Rydbergs. The Fermi energy of 0.5185 Rydbergs was obtained by a fit to the minimum cross section of the $\beta$ arms.

In Figures (V.3), (V.5), (V.7), and (V.9) the open triangles are the experimental dHvA frequencies measured by Poulsen. The solid curves are the corresponding theoretical frequencies. In Figures (V.6), (V.8), and (V.10) the dotted curve shows the cyclotron masses calculated by poulsen using a ten parameter fit. The solid curves are the theoretical masses in the 'rigid band' approximation.

In Figure (V.4) the two experimental masses shown for comparison were measured by Brandt and Rayne. 



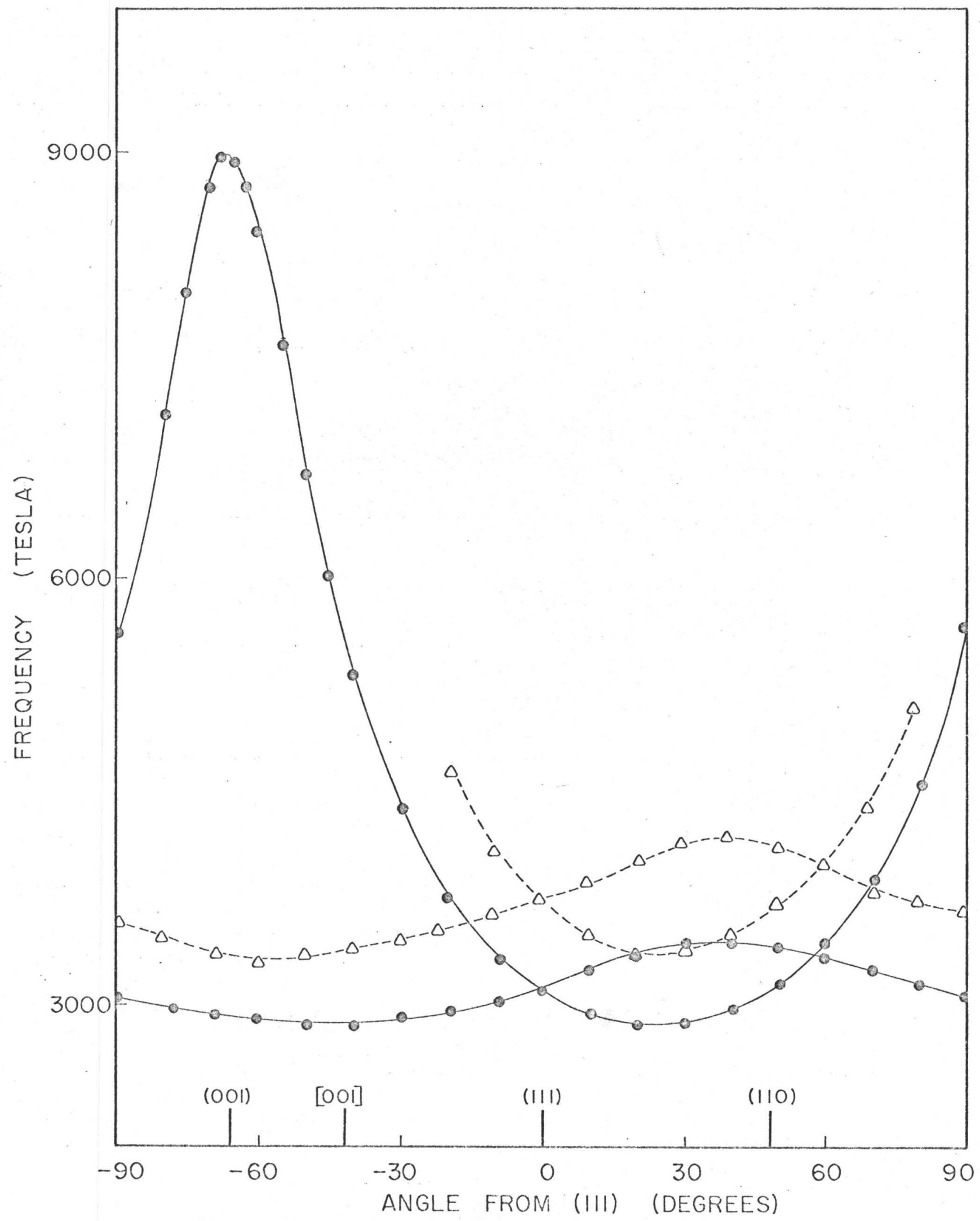

Figure (V.5) Second zone lens dHvA frequencies, trigonalbisectrix plane 


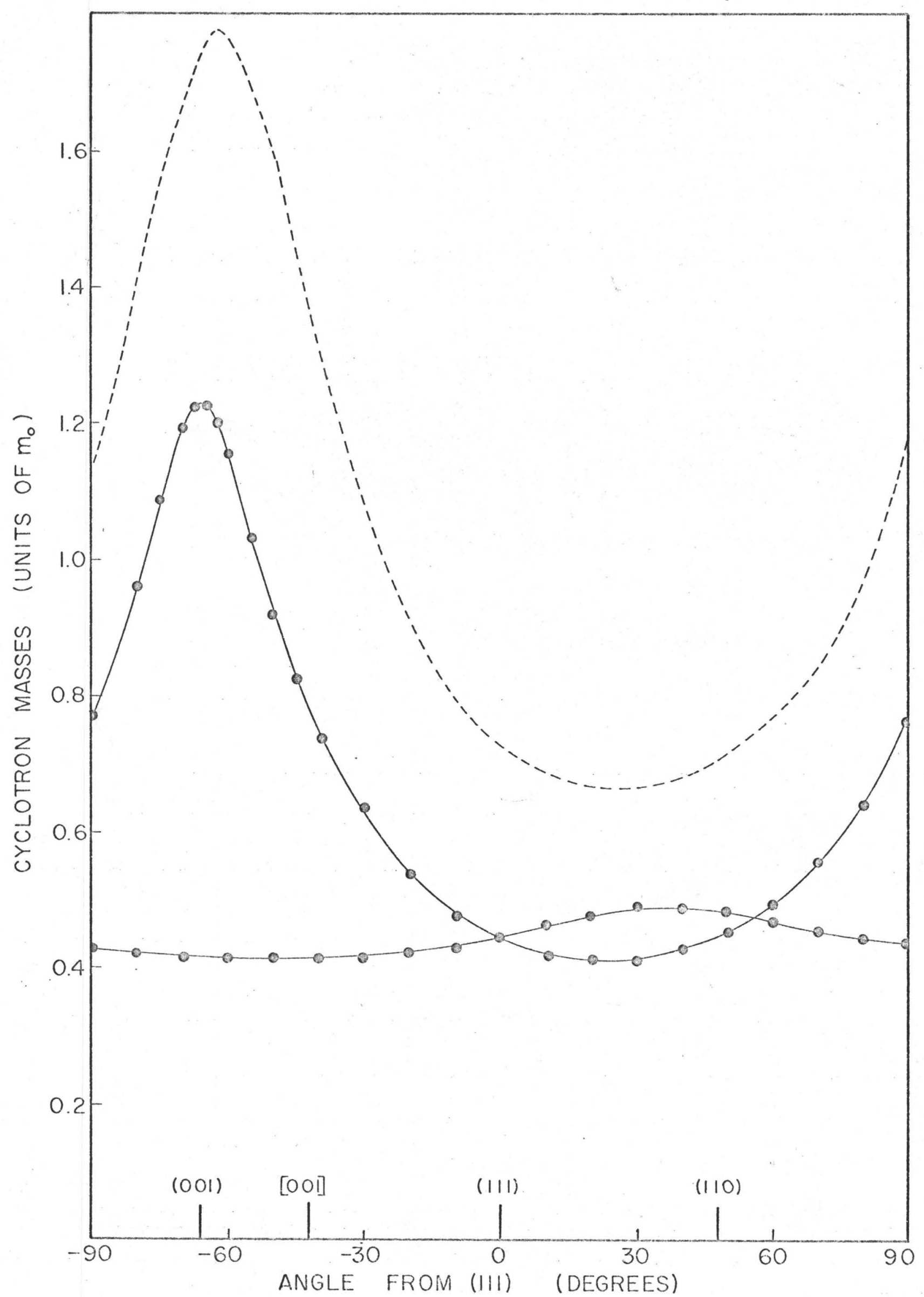

112 


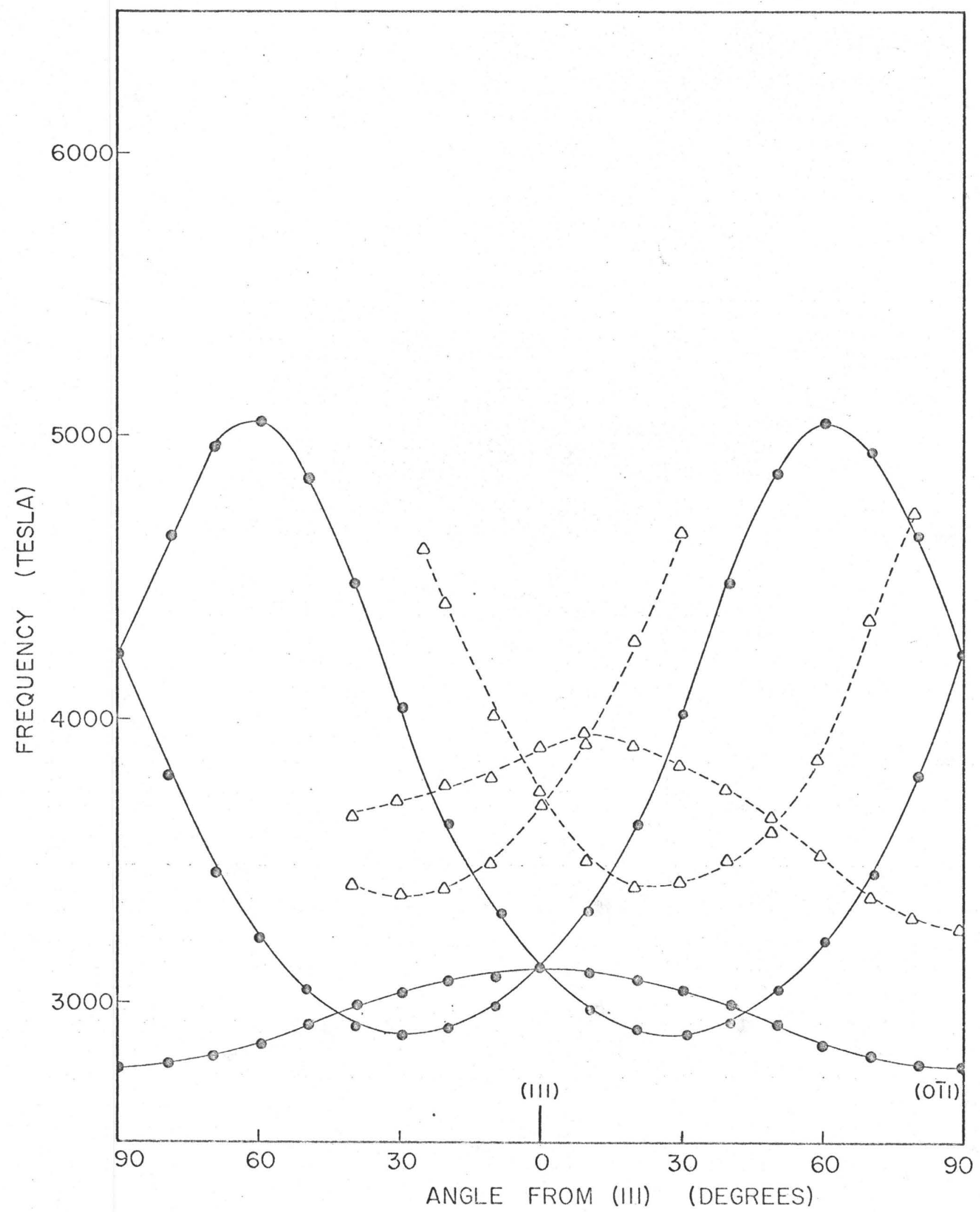

Figure (V.7) Second zone lens dHvA frequencies, trigonalbinary plane. 


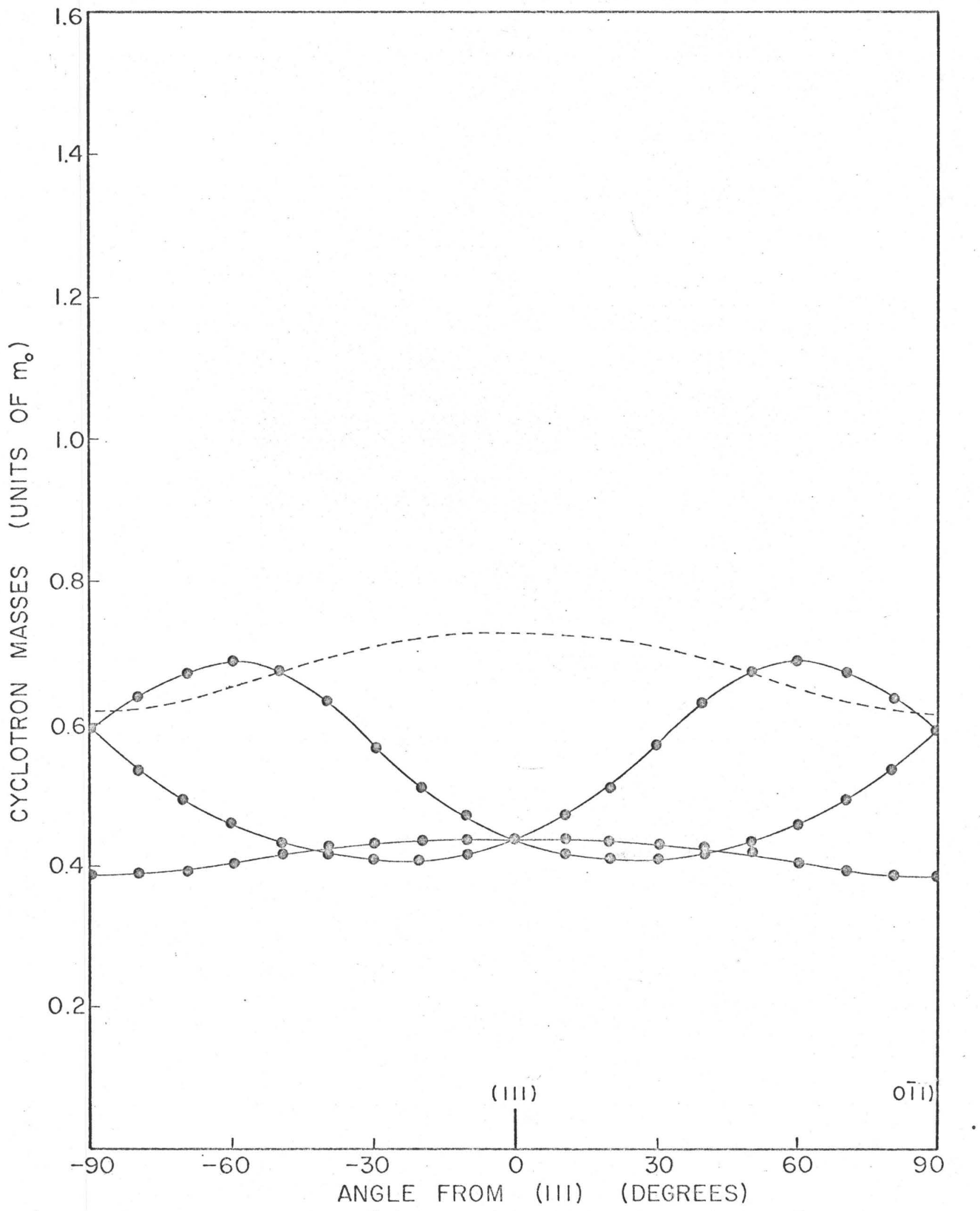

Figure (V.8) Second zone lens cyclotron masses, trigonalbinary plane 


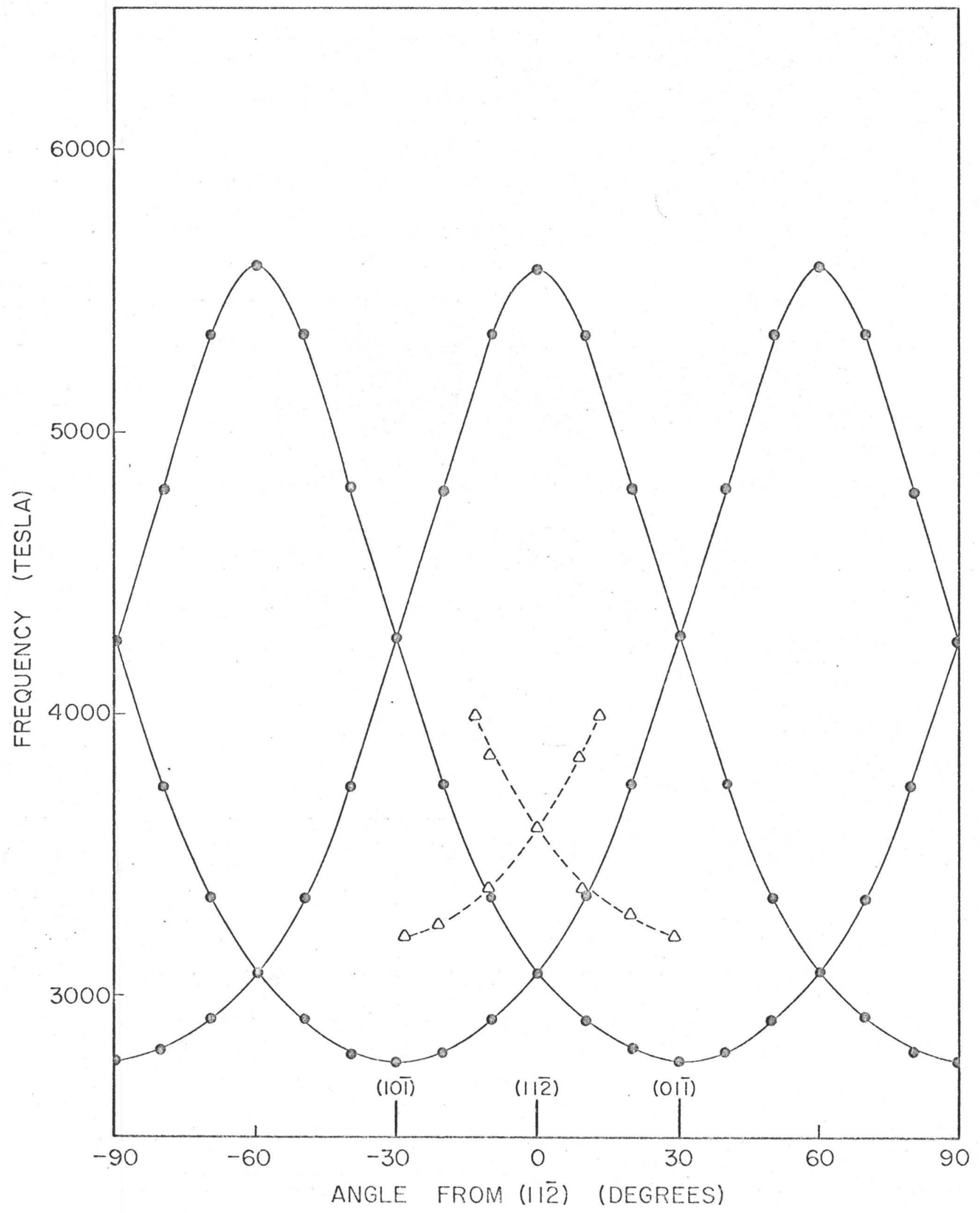

Figure (V.9) Second zone lens dHvA frequencies, trigonal plane. 


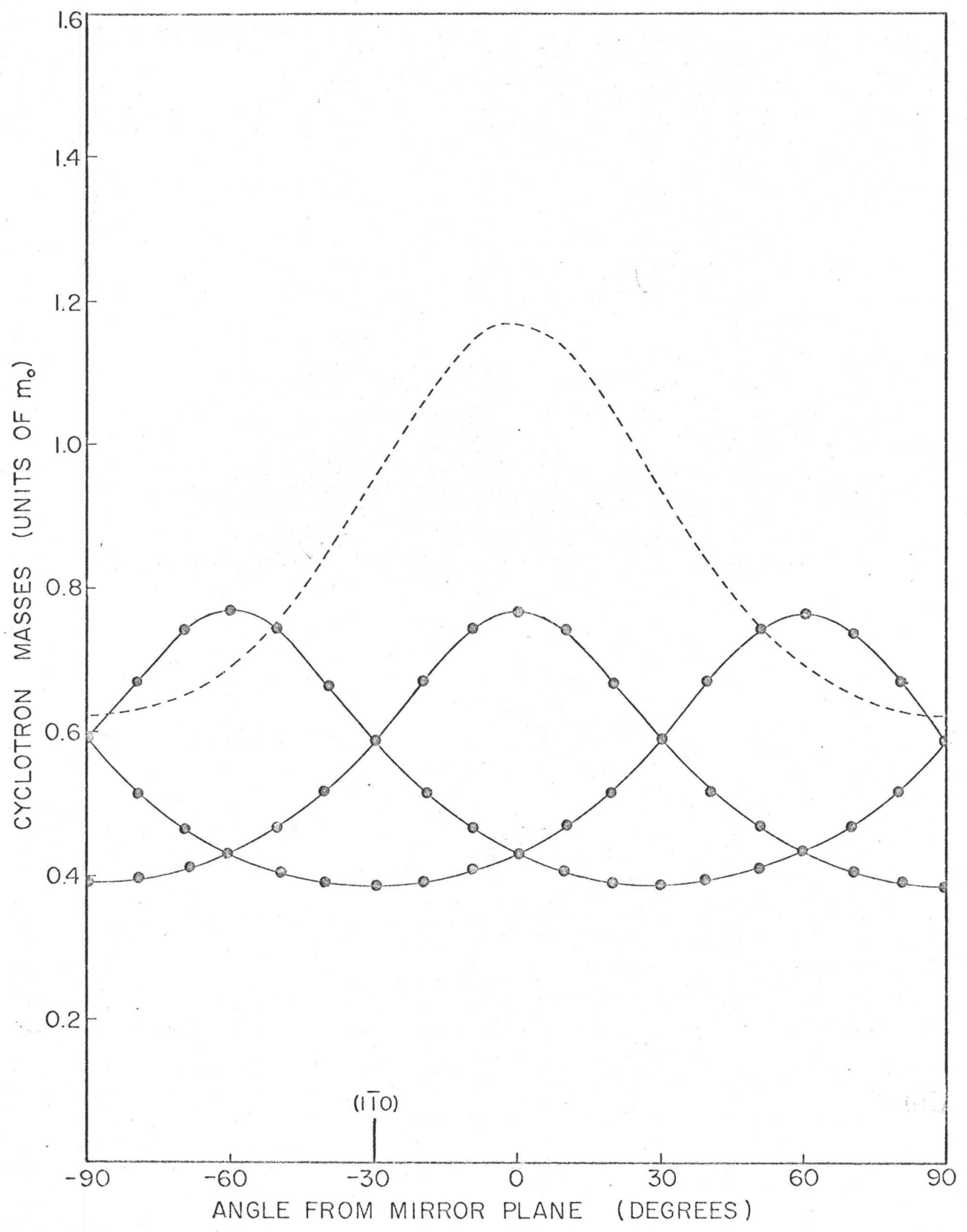

Figure (V.10) Second zone lens cyclotron masses, trigonal plane. 
It is clear that the calculated cross sections of the lenses are too small. The minimum cross section in the trigonal-bisectrix plane has been measured at 3410 Tesla, whereas the calculated value is 2850 Tesla. This was obtained for a Fermi energy of 0.5185 Rydbergs. When the Fermi energy of 0.5243 Rydbergs was used the minimum period came to 3200 Tesla.

These calculations were carried out using secular equations with an average size of $(15 \times 15)$. Subsequent calculations using non-local pseudopotentials usually made use of larger secular equations, up to order $(40 \times 40)$. This involved a considerable increase in the amount of computer time required. For this reason these calculations were limited to extremal areas in the symmetry planes, or in the case of the $\beta$ arms to the minimum cross section.

\section{Three Regions of Parameter Space}

Three regions of parameter space which were of interest were discovered. These will be called pseudopotentials of Class A, Class B, and Class $C$. In each case the pseudohamiltonian was of the form given in equation (4.3)

$$
H_{p}=T+W+R 5 S|5 s\rangle\langle 5 s|+R 5 P| 5 p\rangle\langle 5 p|+R 5 D| 5 d\rangle\langle 5 d| .
$$

In class $A$ and class $C$, R5S was equal to zero and the only local parameters were $\mathrm{W}_{L}$ and $\mathrm{W}_{\mathrm{XT}}$. In the class $\mathrm{B}$ pseudopotentials the $5 \mathrm{~s}$ non-local operator was included and $W$ was given a parameterized form. 
Table (V.3) shows the regions of parameter space which gave the best fits to the 8 points with Class A pseudopotentials. In this and in subsequent similar tables $E_{F}$ is an estimated value for the Fermi energy based on an average taken at the calculated points.

\section{TABLE (V.3)}

\section{Class A Pseudopotentials (8 Point Fits)}

The values of the local pseudopotential parameters $W_{L}, W_{X T}$ which gave the best fits using the 8 point criterion are listed for various values of R5P and R5D, (a.ll energies are in Rydbergs).

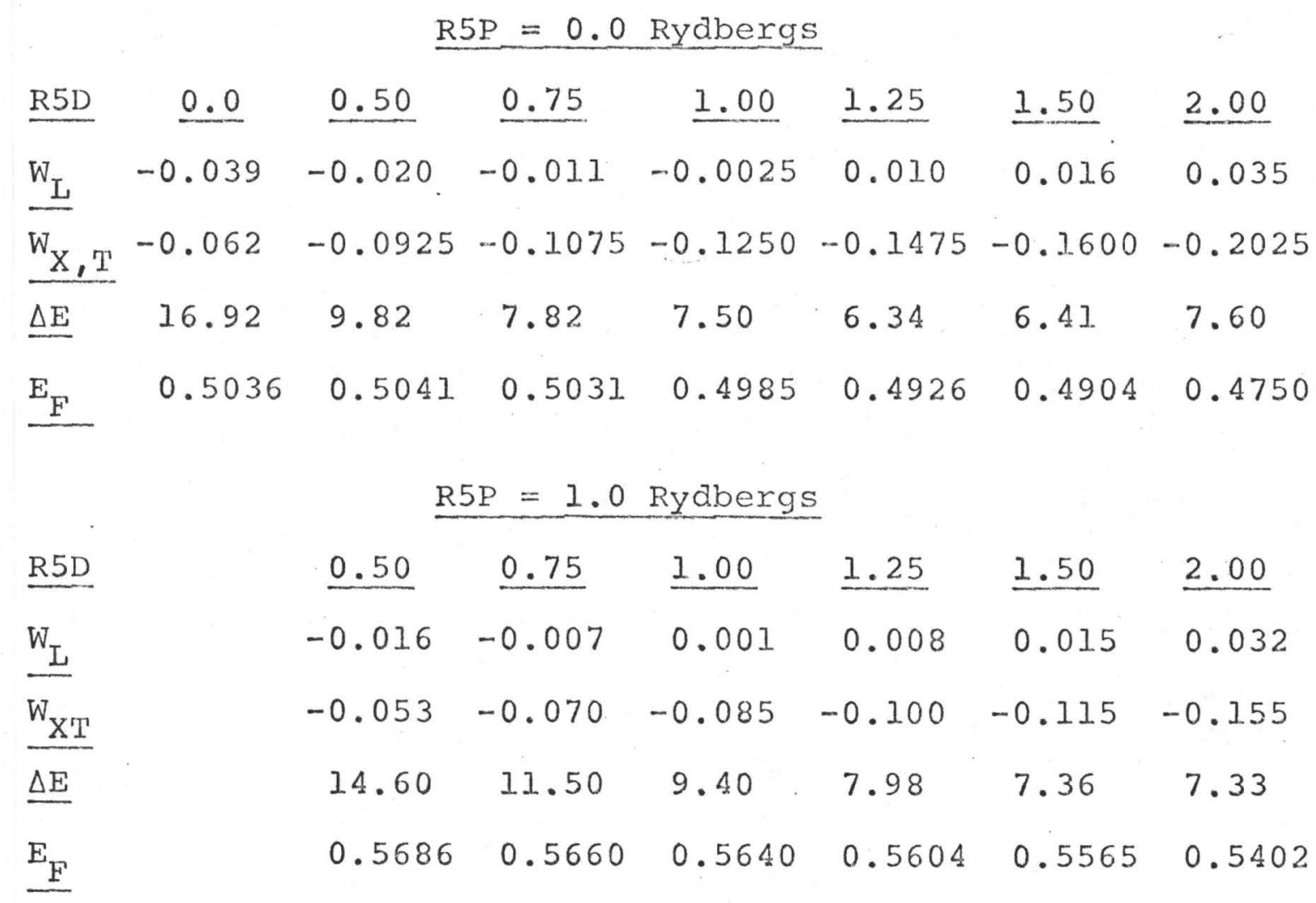


TABLE (V.3) (continued)

$$
\underline{R} 5 \mathrm{P}=2.0 \text { Rydbergs }
$$

\begin{tabular}{|c|c|c|c|c|c|c|}
\hline R5D & 0.50 & 0.75 & 1.00 & 1.25 & 1.50 & 2.00 \\
\hline $\mathrm{w}_{\mathrm{L}}$ & -0.014 & -0.007 & 0.000 & 0.008 & 0.016 & 0.031 \\
\hline $\mathrm{W}_{\mathrm{XT}}$ & -0.027 & -0.040 & -0.054 & -0.070 & -0.087 & -0.125 \\
\hline$\underline{\Delta E}$ & 18.10 & 14.20 & 11.20 & 9.60 & 8.52 & 7.92 \\
\hline $\mathrm{E}_{\mathrm{F}}$ & 0.6097 & 0.6097 & 0.6081 & 0.6045 & 0.5988 & 0.5828 \\
\hline \multicolumn{7}{|c|}{$R 5 P=3.0$ Rydbergs } \\
\hline R.51 & 0.50 & $\underline{0.75}$ & 1.00 & 1.25 & 1.50 & 2.00 \\
\hline $\mathrm{W}_{\text {I }}$ & -0.012 & -0.004 & 0.002 & 0.009 & 0.016 & 0.0285 \\
\hline $\mathrm{w}_{\mathrm{X}}$ & -0.007 & -0.0225 & -0.036 & -0.050 & -0.0675 & -0.098 \\
\hline$\Delta E$ & 20.9 & 16.47 & 13.20 & 10.90 & 9.65 & 8.52 \\
\hline$E_{E}$ & 0.6390 & 0.6382 & 0.6369 & 0.6347 & 0.6282 & 0.6713 \\
\hline
\end{tabular}

$$
\underline{\mathrm{R} 5 \mathrm{P}}=4.0 \text { Rydbergs }
$$

\begin{tabular}{|c|c|c|c|c|c|c|}
\hline R5D & 0.50 & 0.75 & 1.00 & 1.25 & 1.50 & 2.00 \\
\hline $\mathrm{W}_{\mathrm{L}}$ & -0.010 & -0.003 & 0.003 & 0.011 & 0.017 & 0.029 \\
\hline $\mathrm{W}_{\mathrm{XT}}$ & 0.005 & -0.0075 & -0.021 & -0.0375 & -0.0525 & -0.0825 \\
\hline$\underline{\Delta E}$ & 23.1 & 18.1 & 14.5 & 12.0 & 10.3 & 8.9 \\
\hline$E_{F}$ & 0.6583 & 0.6600 & 0.6591 & 0.6553 & 0.6508 & 0.6402 \\
\hline
\end{tabular}

$$
\underline{\text { RP } 5=5.0 \text { Rydbergs }}
$$

$\underline{\text { R5D }} \quad \underline{0.50} \quad \underline{0.75} \quad \underline{1.00} \quad \underline{1.25} \quad \underline{1.50} \quad \underline{2.00}$

$\begin{array}{lcccccc}\mathrm{W}_{\mathrm{L}} & -0.007 & -0.001 & 0.005 & 0.011 & 0.017 & 0.029 \\ \frac{\mathrm{W}_{\mathrm{XT}}}{\underline{\Delta E}} & 0.015 & 0.0025 & -0.010 & -0.025 & -0.040 & -0.070 \\ \underline{\mathrm{E}_{\mathrm{F}}} & 24.6 & 19.5 & 15.6 & 12.8 & 11.0 & 9.35 \\ & 0.6739 & 0.6758 & 0.6761 & 0.6728 & 0.6685 & 0.6578\end{array}$


In class $A$ the lowest energy bands between $\mathrm{X}$ and $\mathrm{K}$ were below the Fermi energy and there were no $\beta$ arms, also the extreme flatness of the energy bands in this region implied heavy cyclotron masses for the $\beta$ orbits, whereas these masses are known to be light. This defect of class A extended over the entire range indicated in Table (V.3). Figures (V.II) and (V.I2) show the band structure for one of these pseudopotentials.

The investigation of this region of parameter space was repeated using the 14 point criterion. These results are shown in Table (V.4).

\section{TABLE (V. 4)}

\section{Class A Pseudopotential (14 Point Fits)}

The values of the local pseudopotential parameters $W_{L}, W_{X T}$ which gave the best fits using the 14 point criterion are listed for various values of $\mathrm{R} 5 \mathrm{P}$ and $\mathrm{R} 5 \mathrm{D}$. (All energies are in Ry.dbergs) .

$$
\underline{R} 5 \mathrm{P}=0.0 \text { Rydbergs }
$$

\begin{tabular}{|c|c|c|c|c|c|c|c|}
\hline R5 $\mathrm{D}^{\circ}$ & 0.0 & 0.50 & 0.75 & 1.00 & 1.25 & 1.50 & 2.00 \\
\hline $\mathrm{W}_{\mathrm{L}}$ & -0.044 & -0.029 & -0.024 & -0.019 & -0.013 & -0.010 & 0.00 \\
\hline $\mathrm{W}_{\mathrm{XT}}$ & -0.0470 & -0.0700 & -0.0775 & -0.0850 & -0.0950 & -0.1000 & -0.1175 \\
\hline$\Delta \mathrm{E}$ & 20.34 & 19.55 & 20.11 & 20.93 & 21.84 & 22.75 & 24.44 \\
\hline$F$ & 0.5166 & 0.5228 & 0.5261 & 0.5286 & 0.5290 & 0.5313 & 0.5307 \\
\hline
\end{tabular}


TABLE (V.4) (continued)

$$
R 5 P=1.0 \text { Rydbergs }
$$

\begin{tabular}{|c|c|c|c|c|c|c|c|}
\hline R5D & 0.0 & 0.50 & 0.75 & 1.00 & 1.25 & 1.50 & 2.00 \\
\hline $\mathrm{W}_{\mathrm{L}}$ & -0.035 & -0.022 & -0.017 & -0.013 & -0.007 & -0.004 & 0.004 \\
\hline $\mathrm{w}_{\mathrm{XT}}$ & -0.0125 & -0.035 & -0.0425 & -0.050 & -0.060 & -0.065 & -0.080 \\
\hline$\Delta E$ & 24.43 & 21.73 & 21.95 & 22.69 & 23.73 & 24.84 & 26.99 \\
\hline$E_{F}$ & 0.5769 & 0.5851 & 0.5891 & 0.5919 & 0.5927 & 0.5955 & 0.5963 \\
\hline \multicolumn{8}{|c|}{$\mathrm{R} 5 \mathrm{P}=2.0$ Rydbergs } \\
\hline$\underline{\mathrm{R} 5 \mathrm{D}}$ & 0.00 & $\underline{0.50}$ & 0.75 & 1.00 & 1.25 & 1.50 & $\underline{2.00}$ \\
\hline $\mathrm{W}_{\mathrm{L}}$ & -0.029 & -0.018 & -0.012 & -0.008 & -0.004 & 0.00 & 0.008 \\
\hline $\mathrm{W}_{\mathrm{XT}}$ & 0.0125 & -0.0100 & -0.0200 & -0.0275 & -0.035 & -0.0425 & -0.0575 \\
\hline$\Delta \mathrm{E}$ & 27.54 & 23.26 & 23.07 & 23.64 & 24.69 & 25.93 & 28.49 \\
\hline$E_{F}$ & 0.6152 & 0.6256 & 0.6287 & 0.6321 & 0.6345 & 0.6360 & 0.6372 \\
\hline & & \multicolumn{3}{|c|}{$\mathrm{R} 5 \mathrm{P}=3.0$ Rydbergs } & \multicolumn{3}{|c|}{. } \\
\hline$\underline{\mathrm{R} 5 \mathrm{D}}$ & 0.00 & 0.50 & 0.75 & 1.00 & 1.25 & 1.50 & 2.00 \\
\hline $\mathrm{W}_{\mathrm{L}}$ & -0.024 & -0.014 & -0.009 & -0.004 & -0.001 & 0.004 & 0.010 \\
\hline $\mathrm{W}_{\mathrm{XT}}$ & 0.030 & 0.006 & -0.003 & -0.012 & -0.018 & -0.0275 & -0.040 \\
\hline$\Delta \mathrm{E}$ & 29.78 & 24.45 & 23.79 & 24.19 & 25.20 & 26.51 & 29.30 \\
\hline$E_{F}$ & 0.6408 & $\begin{array}{l}0.6525 \\
\mathrm{R} 5 \mathrm{P}\end{array}$ & $\begin{array}{r}0.6566 \\
=4.0 \mathrm{Ry} \\
\end{array}$ & 0.6594 & 0.6630 & 0.6636 & 0.6665 \\
\hline$\underline{\mathrm{R} 5 \mathrm{D}}$ & 0.00 & 0.50 & 0.75 & 1.00 & 1.25 & 1.50 & 2.00 \\
\hline $\mathrm{W}_{\mathrm{L}}$ & -0.019 & -0.010 & -0.006 & -0.001 & 0.002 & 0.006 & 0.013 \\
\hline $\mathrm{W}_{\mathrm{XT}}$ & 0.0425 & 0.018 & 0.009 & 0.00 & -0.0075 & -0.015 & -0.030 \\
\hline$\Delta \mathrm{E}$ & 31.37 & 25.37 & 24.33 & 24.52 & 25.46 & 26.77 & 29.78 \\
\hline $\mathrm{E}_{\mathrm{F}}$ & 0.6585 & 0.6718 & 0.6765 & 0.6795 & 0.6825 & 0.6844 & 0.6860 \\
\hline
\end{tabular}


TABLE (V.4) (continued)

$$
\underline{R} 5 \mathrm{P}=5.0 \text { Rydbergs }
$$

\begin{tabular}{|c|c|c|c|c|c|c|c|}
\hline R5D & 0.00 & 0.50 & 0.75 & 1.00 & 1.25 & 1.50 & 2.00 \\
\hline $\mathrm{W}_{\mathrm{I}}$ & -0.015 & -0.007 & -0.003 & 0.001 & 0.004 & 0.008 & 0.014 \\
\hline $\mathrm{W}_{\mathrm{XT}}$ & 0.0525 & 0.0275 & 0.0175 & 0.009 & 0.0025 & -0.006 & -0.018 \\
\hline$\Delta \mathrm{E}$ & 32.48 & 25.98 & 24.73 & 24.71 & 25.98 & 26.90 & 30.05 \\
\hline$s_{F}$ & 0.6718 & 0.6865 & 0.6911 & 0.6947 & 0.6985 & 0.6999 & 0.7037 \\
\hline
\end{tabular}

For a given value of $\mathrm{R} 5 \mathrm{P}$ and $\mathrm{R} 5 \mathrm{D}$ there are quite laxge changes in the values of $W_{L}$ and $W_{X T}$ when changing from the best 8 point fit to the best 14 point fit. The reason for this is that if contour lines of equally good fit are drawn in the $W_{L}, W_{X T}$ plane it is found that the region of best fit is a very elongated valley with almost equally good fits occurring along a considerable length. For example for $\mathrm{R} 5 \mathrm{P}=2.0$, and $\mathrm{R} 5 \mathrm{D}=1.0$ Rydbergs, $\Delta \mathrm{E}(8$ point $)$ assumed the following values at two points along the axis of the valley,

$$
\begin{aligned}
& \mathrm{W}_{L_{1}}=0.03, \mathrm{~W}_{\mathrm{XT}}=-0.060, \Delta \mathrm{E}=11.65, \mathrm{E}_{\mathrm{F}}=0.6042 \text { Ryd. } \\
& \mathrm{W}_{\mathrm{L}}=-0.02, \mathrm{~W}_{\mathrm{XT}}=-0.048, \Delta \mathrm{E}=12.00, \mathrm{E}_{\mathrm{F}}=0.6127 \text { Ryd. }
\end{aligned}
$$

The best 8 point fit reported in Table (V.3) for this value of $\mathrm{R} 5 \mathrm{P}$ and $\mathrm{R} 5 \mathrm{D}$ was at the point

$$
\mathrm{W}_{\mathrm{L}}=0.00, \mathrm{~W}_{\mathrm{XT}}=-0.054, \Delta \mathrm{E}=11.50, \mathrm{E}_{\mathrm{F}}=0.6081 .
$$

By comparison the best 14 point fit for this value of $R 5 P$ and R5D was given by

$$
\mathrm{W}_{\mathrm{L}}=-0.008, \mathrm{~W}_{\mathrm{XT}}=-0.0275, \Delta \mathrm{E}=23.64, \mathrm{E}_{\mathrm{F}}=0.6321 .
$$




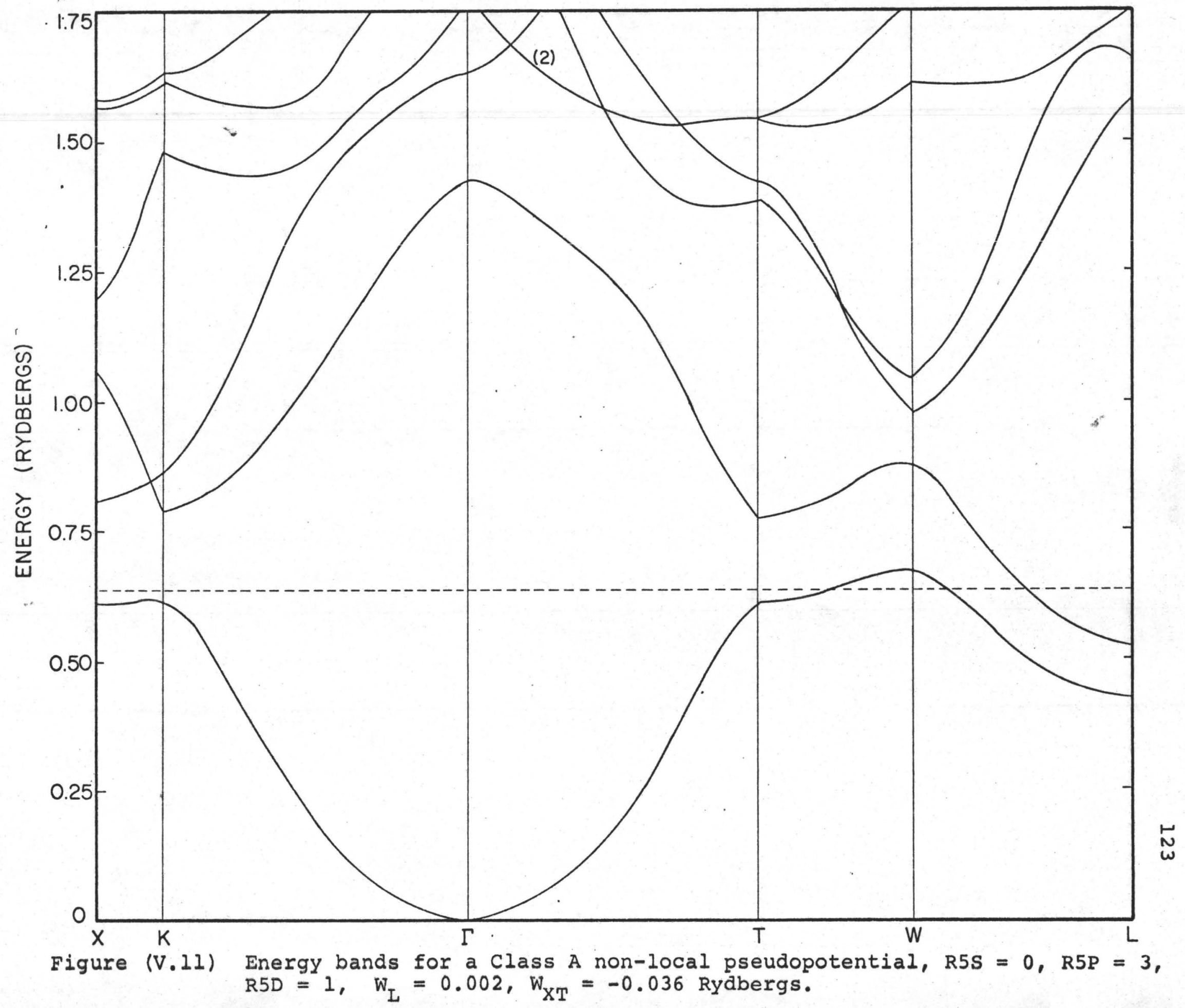







The reason for the large shift in the parameters is that the 14 point fit reached an optimum value further along the elongated minimum. It was found that the original defects remained, the $\beta$ arms did not appear and the lowest bandsalong the XK line were extremely flat.

In Table (V.4) there is a tendency for $\Delta \mathrm{E}$ to reach a minimum value somewhere near $\mathrm{R} 5 \mathrm{D}=0.75$ Rydbergs, which is near the value which would be expected in an OPW-type pseudopotential similar to that given in equation (2.7).

With the non-local parameters set at the approximate OPW-values of $R 5 S=\left(E_{6 s}-E_{5 s}\right), R 5 P=\left(E_{6 s}-E_{5 p}\right)$, and $R 5 D=\left(E_{6 s}-E_{5 d}\right)$, (making use of the atomic energy levels of Herman and Skillman) there is a region of parameter space corresponding to the class B pseudopotentials which produces energy bands very similar to the bands generated by the local pseudopotentials such as those of Brandt and Rayne. This set of pseudopotentials generates a Fermi surface having the correct topology, with second zone lenses and first zone contact regions around $\mathrm{x}$ and $\mathrm{T}$. There were also $\beta$ arms.

Using the above values for R5S, R5P, and R5D, and varying $W_{L}$ and $W_{X T}$, the optimum value of $\triangle E$ ccurred at $\mathrm{W}_{\mathrm{L}}=-0.200$ Rydbergs, $\mathrm{W}_{\mathrm{XT}}=-0.140$ Rydbergs, with a Fermi energy relative to the bottom of the band of 0.5760 Rydbergs, . and a 14 point figure of merit of 29. 
A parameterized form for the local pseudopotential was also used in which $W(q)$ was given by the four parameter empirical approximation

$$
W(q)=\alpha\left(q^{2}-\beta\right) /\left[\exp \left(\gamma\left(q^{2}-\delta\right)\right)+1\right] .
$$

Using this parameterized form it was quite easy to find values of $\alpha, \beta, \gamma$, and $\delta$ which gave 14 point figures of merit of about 20 .

An example of a band structure generated by such a pseudopotential is shown in Figures (V.13) and (V.14), for which,

$$
\alpha=0.07, \beta=3.5, \gamma=1.5, \text { and } \delta=4.0 \text {. }
$$

These parameters gave $\mathrm{W}_{\mathrm{L}}=-0.138, \mathrm{~W}_{\mathrm{X}}=-0.083$, and $\mathrm{W}_{\mathrm{T}}=$ -0.082 Rydbergs.

A comparison of the energy bands with those of Brandt and Rayne illustrated in Figures (III.4) and (III.5), and with those shown in Figures (V.I) and (V.2) for the best two parameter local pseudopotential ( 8 point criterion), show strong qualitative resemblances. There was an important difference, whereas the local pseudopotentials just mentioned have second zone lenses that are essentially p-like, the class B pseudopotentials have lenses of predominantly 's-d' symmetry.

At the present time there appear to have been no experimental investigations to determine the general symmetry character of the states responsible for the second zone lenses. 


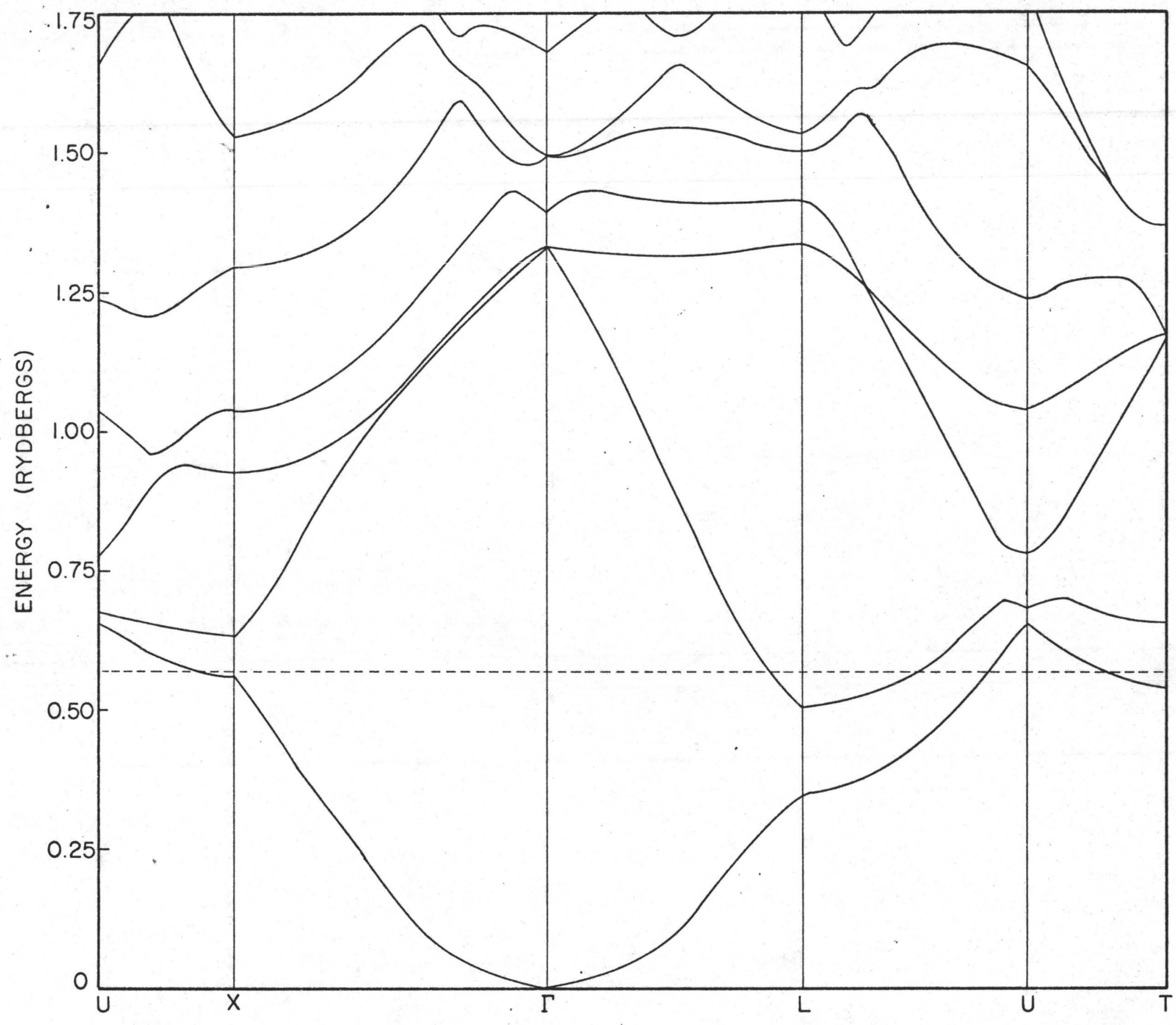

Figure (V.14) Energy bands for a class $B$ non-local pseudopotential, $\alpha=0.07, \beta=3.5$, $\gamma=1.5, \delta=4.0$ 
Table (V.5) shows the variation of $\Delta \mathrm{E}$ (14 point figure of merit) as R5S, R5P, and R5D were varied in turn and all other parameters were held fixed. Three new parameters CS, CP, and $C D$ were introduced and the non-local parameters were given by,

$$
\begin{aligned}
& \text { R5S }=C S \times\left(E_{6 s}-E_{5 s}\right), R 5 P=C P \times\left(E_{6 s}-E_{5 p}\right), \\
& \text { R5D }=C D \times\left(E_{6 s}-E_{5 d}\right) .
\end{aligned}
$$

In the example shown $\beta, \gamma$, and $\delta$ were held fixed at the values

$$
\beta=2.5, \gamma=1.5, \delta=4.0 \text {. }
$$

For each set of parameters CS, CP, and CD, a was varied until $\Delta \mathrm{E}$ reached a minimum.

\section{TABLE $(V .5)$}

\section{A Set of Class B Pseudopotentials}

The variation of the 'best fit to 14 points' of one of the parameterized pseudopotentials, as a function of the strength of the $5 \mathrm{~s}, 5 \mathrm{p}$, and $5 \mathrm{~d}$ non-local operators $\mathrm{R} 5 \mathrm{~S}=\mathrm{CS} \times\left(\mathrm{E}_{6 \mathrm{~s}}-\mathrm{E}_{5 \mathrm{~s}}\right)$, $R 5 P=C P \times\left(E_{6 s}-E_{5 p}\right), R 5 D=C D \times\left(E_{6 s}-E_{5 d}\right)$, where $E_{5 s^{\prime}}, E_{5 p^{\prime}}$ $\mathrm{E}_{5 \mathrm{~d}}$, and $\mathrm{E}_{6 \mathrm{~s}}$ are the corresponding atomic energy levels as computed by Herman and SkiJlman. The local pseudopotential is of the form

$$
W(q)=\alpha\left(q^{2}-\beta\right) /\left[\exp \gamma\left(q^{2}-\delta\right)+1\right]
$$

In this nodel $\beta=2.5, \gamma=1.5, \delta=4.0$, all energies are in Rydbergs. 
TABLE (V.5) (continued)

Varying the strength of the 5 s projection operator

\begin{tabular}{|c|c|c|c|c|c|}
\hline CS & $\mathrm{CP}$ & $\mathrm{CD}$ & $\alpha$ (giving best fit) & $\begin{array}{l}\bar{E} \text { (mean energy } \\
\text { of } 14 \text { points })\end{array}$ & $\Delta \mathrm{E}$ (1.4points) \\
\hline 0.5 & J. & 1. & 0.0225 & 0.6193 & 33.00 \\
\hline 0.7 & 1. & 1. & 0.0375 & 0.6033 & 22.39 \\
\hline 0.8 & 1. & 1. & 0.0475 & 0.5964 & 20.97 \\
\hline 0.9 & 1. & 1. & 0.0600 & 0.5899 & 20.89 \\
\hline 1. & 1. & 1. & 0.0650 & 0.5838 & 20.79 \\
\hline 1.1 & 1. & 1. & 0.0700 & 0.5783 & 21.80 \\
\hline 1.3 & 1 . & 1. & 0.0850 & 0.5678 & 24.36 \\
\hline 1.5 & 1. & 1. & 0.0 .920 & 0.5588 & 27.60 \\
\hline
\end{tabular}

Varying the strength of the $5 p$ projection operator

$\begin{array}{llllcc}\text { CS } & \text { CP } & \underline{C D} & \underline{\alpha} & \underline{E} & \underline{\Delta E} \\ 1 . & 0.8 & 1 . & 0.0825 & 0.5642 & 31.04 \\ 1 . & 0.9 & 1 . & 0.0700 & 0.5755 & 24.40 \\ 1 . & 1 . & 1 . & 0.0650 & 0.5838 & 20.79 \\ 1 . & 1.1 & 1 . & 0.0600 & 0.5908 & 20.09 \\ 1 . & 1.2 & 1 . & 0.0550 & 0.5965 & 21.29 \\ 1 . & 1.3 & 1 . & 0.0500 & 0.6013 & 23.52 \\ 1 . & 1.5 & 1 . & 0.0450 & 0.6091 & 28.68\end{array}$

Varying the strength of the $5 \mathrm{~d}$ projection operator

$\begin{array}{lllccc}\mathrm{CS} & \underline{C P} & \underline{C D} & \underline{\alpha} & \underline{E} & \underline{E} \\ 1 . & 1 . & 0 & 0.0650 & 0.5038 & 27.49 \\ 1 . & 1 . & 0.5 & 0.0625 & 0.5492 & 26.85\end{array}$


TABLE (V.5) (continued)

$\begin{array}{llllcl}\mathrm{CS} & \underline{\mathrm{CP}} & \underline{\mathrm{CD}} & \underline{\alpha} & \overline{\mathrm{E}} & \underline{\Delta \mathrm{E}} \\ \text { 1. } & 1 . & 0.6 & 0.0625 & 0.5565 & 23.17 \\ 1 . & 1 . & 0.7 & 0.0625 & 0.5637 & 20.79 \\ 1 . & 1 . & 0.8 & 0.0650 & 0.5707 & 19.57 \\ 1 . & 1 . & 0.9 & 0.0650 & 0.5773 & 19.58 \\ 1 . & 1 . & 1 . & 0.0650 & 0.5838 & 20.79 \\ 1 . & 1 . & 1 . & 0.0650 & 0.5902 & 23.00 \\ 1 . & 1 . & 1.3 & 0.0675 & 0.6020 & 29.13 \\ 1 . & 1 . & 1.5 & 0.0700 & 0.6131 & 36.37\end{array}$

The class $C$ pseudopotentials generated a Fermi surface of the correct topology and $\beta$ arms were present. Table (V.6) shows the regions of parameter space which gave the best 14 point fits.

\section{TABLE $(V .6)$}

\section{Class C Pseudopotentials}

The values of the local pseudopotential parameters $W_{L}, W_{X T}$ which gave the best fits using the 14 point criterion are listed for various values of R5P and R5D. (All energies are in Rydbergs).

\begin{tabular}{|c|c|c|c|c|c|}
\hline R5D & 0.00 & 0.50 & 1.00 & 2.00 & 3.00 \\
\hline $\mathrm{W}_{\mathrm{L}_{\mathrm{I}}}$ & -0.082 .5 & -0.0775 & -0.0725 & -0.0625 & -0.055 \\
\hline $\mathrm{w}_{\mathrm{XT}}$ & 0.0475 & 0.0425 & 0.0375 & 0.030 & 0.0225 \\
\hline$\Delta E$ & 20.45 & 23.35 & 27.50 & 36.42 & 44.67 \\
\hline$E_{E}$ & 0.5247 & 0.5443 & 0.5609 & 0.5869 & 0.6058 \\
\hline
\end{tabular}




\section{$\underline{R} 5 \mathrm{P}=0.5$ Rydbergs}

\begin{tabular}{|c|c|c|c|c|c|}
\hline R5D & \multicolumn{2}{|l|}{0.00} & 0.50 & \multicolumn{2}{|c|}{1.00} \\
\hline$\underline{\mathrm{W}_{\mathrm{L}}}$ & \multicolumn{2}{|l|}{-0.070} & -0.0675 & \multicolumn{2}{|c|}{-0.065} \\
\hline $\mathrm{W}_{\mathrm{XT}}$ & \multicolumn{2}{|c|}{0.0725} & 0.0625 & \multicolumn{2}{|c|}{0.0575} \\
\hline$\Delta \mathrm{E}$ & \multicolumn{2}{|l|}{20.13} & 23.72 & \multicolumn{2}{|c|}{28.14} \\
\hline $\mathrm{E}_{\mathrm{F}}$ & \multicolumn{2}{|c|}{0.5512} & 0.5751 & \multicolumn{2}{|c|}{0.5931} \\
\hline \multicolumn{6}{|c|}{$R 5 P=1.0$ Rydbergs } \\
\hline R5D & 0.00 & 0.50 & 1.00 & 1.50 & 2.00 \\
\hline $\mathrm{w}_{\mathrm{L}}$ & -0.0575 & -0.060 & -0.0575 & -0.0525 & -0.0475 \\
\hline $\mathrm{W}_{\mathrm{XT}}$ & 0.0925 & 0.080 & 0.075 & 0.069 & 0.0625 \\
\hline$\underline{\Delta E}$ & 19.52 & 23.84 & 28.68 & 33.28 & 37.69 \\
\hline $\mathrm{E}_{\mathrm{F}}$ & 0.5698 & 0.5977 & 0.6167 & 0.6331 & 0.6471. \\
\hline & & $=2.0 \mathrm{RI}$ & bergs & & \\
\hline R5D & 2.0 & & & & \\
\hline $\mathrm{W}_{\mathrm{L}}$ & -0.0375 & & & & \\
\hline $\mathrm{W}_{\mathrm{XT}}$ & 0.0875 & & & & \\
\hline$\underline{\Delta E}$ & 38.75 & & & & \\
\hline$E_{F}$ & 0.6830 & & & & \\
\hline
\end{tabular}

Table $(V \cdot \dot{7})$ shows some of the extrenal cross-sectional. areas and cyclotron masses $\left(\frac{J}{\pi} \frac{\partial A}{\partial \varepsilon}\right)$ which were calculated for some of the class C pseudopotentials. The Fermi energy was defined by fitting the $\beta$ arms to the experimental frequency of 74 Tesla. 
The extremal cross sections are the 'large section' of the lens, ( $\mathrm{H}$ is the direction $\Gamma L$ ), the 'up section' of the lens, ( $\underline{H}$ in the direction LU), and the 'side section' of the lens (H in the direction $L W$ ). The ' $\tau$ minimum cross section' is the minimum cross sectional area of the $\tau$ orbit, with $\mathrm{H}$ in the (1I0), binary direction. The other two areas given in Table (V.7) refer to the $T$ face and $X$ face holes.

Poulsen obtained a value of 3240 Tesla for the 'side section' of the lens, and 3410 Tesla for the 'up section' The large cross sectional area of the lens has not been measured. Poulsen reported a frequency for the minimum $\tau$ cross section of 1580 Tesla.

The $\mathrm{X}$ and $\mathrm{T}$ face breakthrough regions have not been positively identified in dHvA experiments, although Moss (1968) reported weak signals with $\mathrm{H}$ oriented in the (111) direction at a frequency of 1950 Tesla. These could have come from the T face hole. In Table (V.7) the notation $C(P, D)$ means that $R 5 P=P$ Rydbergs and R5D $=\mathrm{D}$ Rydbergs .

In Table (V.7) the lenses are a little too small but only by a few per cent. There is a consistent tendency for the $\tau$ orbit to be too small and for the $T$ face hole to be large. These two conditions are correlated, a contraction of the $T$ face hole would be accompanied by an increase in the size of the $\tau$ orbits. 
TABLE (V.7)

Computed Values of the de Haas-van Alphen Frequencies and Cyclotron Masses $\left(\frac{1}{\pi} \frac{\partial A}{\partial \varepsilon}\right)$ for Various Non-local Pseudopotentials

Frequencies (Tesla)

\begin{tabular}{|c|c|c|c|c|c|c|c|c|}
\hline $\begin{array}{l}\text { Pseudo- } \\
\text { potential }\end{array}$ & $\begin{array}{l}E_{F} \\
(\text { Ryd.) } \\
\end{array}$ & $\beta_{\text {min. }}$ & $\begin{array}{l}\text { Lens } \\
\text { (large) }\end{array}$ & $\begin{array}{l}\text { Lens } \\
\text { (up) } \\
\end{array}$ & $\begin{array}{l}\text { Lens } \\
\text { (side) }\end{array}$ & $\tau^{\tau} \min$ & $\mathrm{T}_{\text {face }}$ & $x_{f a c t}$ \\
\hline 10 & 0.5 & 74. & 9888 & 3160 & 3052 & 1126 & 4441 & 07 \\
\hline$(0,1)$ & 0.5497 & 74. & 10417 & 3213 & 3026 & 9 & 4 & 69 \\
\hline$=(0$, & 0.5731 & 74. & 10881 & 3172 & 2977 & 1164 & 0 & 27 \\
\hline$(1,0)$ & 0.5606 & 74. & 9905 & 3057 & 3024 & 1257 & 3216 & 1076 \\
\hline$(1,1)$ & 0.6020 & 74.30 & 9765 & 3059 & 2898 & 1228 & 4 & 2476 \\
\hline$(1,2)$ & 0.6312 & 74.26 & 10452 & 3135 & 2969 & 1258 & 3463 & 2898 \\
\hline
\end{tabular}

\section{Effective Masses (reduced units).}

\begin{tabular}{|c|c|c|c|c|c|c|c|c|}
\hline $\begin{array}{l}\text { Pseudo- } \\
\text { potential }\end{array}$ & $\underset{(\mathrm{Ry} \mathrm{d} .)}{\mathrm{E}_{\mathrm{F}}}$ & $\beta_{\min }$ & $\begin{array}{l}\text { Lens } \\
\text { large }\end{array}$ & $\begin{array}{l}\text { Lens } \\
\text { (up) }\end{array}$ & $\begin{array}{l}\text { Lens } \\
\text { (side) }\end{array}$ & ${ }^{\tau} \min$ & $\mathrm{T}_{\text {face }}$ & $\mathrm{X}_{\text {face }}$ \\
\hline$c(0,1)$ & 168 & .0812 & 1.2166 & 0.4078 & 0.3881 & -0.3231 & .2235 & 1.092 \\
\hline $\mathrm{ClO}$ & 0.5497 & -0.0627 & 0.8932 & 0.3140 & 0.2912 & -0.2436 & 7953 & 7013 \\
\hline$C(0$ & 0.5731 & -0.0588 & 687 & 0.2833 & 0.2606 & -0.2218 & 6310 & 0 \\
\hline$C(]$ & 0.5606 & -0.0833 & 1.3350 & 0.4256 & 0.4123 & 596 & 3445 & 1.1945 \\
\hline$C(1$ & 0.6020 & -0.0591 & 0.8903 & 0.3044 & 0.2841 & -0.2450 & 0.8036 & 0.7055 \\
\hline$C(1,2)$ & 0.6471 & -0. & 10. & & 0.2 & -0 . & 0.6131 & 0.547 \\
\hline
\end{tabular}


The rather wide fluctuations in the calculated sizes of the $X$ and $T$ face holes can be understood in terms of the flat gradient (related to the high effective mass) of the constant energy surfaces in the region of these faces. This means that a small change in the value chosen for $\mathrm{E}_{\mathrm{F}}$ will produce quite a large change in the areas of these openings.

The diagrams shown in Figures (V.15), ..., (V.21) show various sections of the Fermi surface for the local pseudopotential $\mathrm{C}(0,0)$. In this model $\mathrm{W}_{\mathrm{L}}=-0.0825$ Rydbergs, $\mathrm{W}_{\mathrm{XT}}=0.0475$ Rydbergs, and the Fermi energy was equal to 0.5168 Rydbergs. These diagrams were constructed using the output of the computer programs that calculated the cross sectional areas and the cyclotron masses, the order of the secular equations varied between $(33 \times 33)$ and $(40 \times 40)$ according to the position in the Brillouin zone.

These cross sections resemble similar figures which have been given by Brandt and Rayne (1966). The outer edge of the $\beta$ arm shown in Figure (V.15) is inset from the edge of the $X$ face, as was first predicted by the RAPW calculations of Keeton and Loucks (1966).

Table (V.8) shows the set of extremal orbits for the class B pseudopotential whose band structure is illustrated in Figures (V.13) and (V.I.4). There was some difficulty with convergence when calculating the cross sectional areas for this class of pseudopotentials and the figures given are 
Figures (V.15), ..., (V.2I) show different computed cross sections of the Fermi surface for the local pseudopotential $\mathrm{C}(\mathrm{O}, \mathrm{O})$ for which $\mathrm{W}_{\mathrm{L}}=-0.0825, \mathrm{~W}_{\mathrm{XT}}=0.0475$ Rydbergs, the Fermi energy has been set equal to 0.5168 Rydbergs by fitting to the experimental minimum $\beta$ arm cross section. 


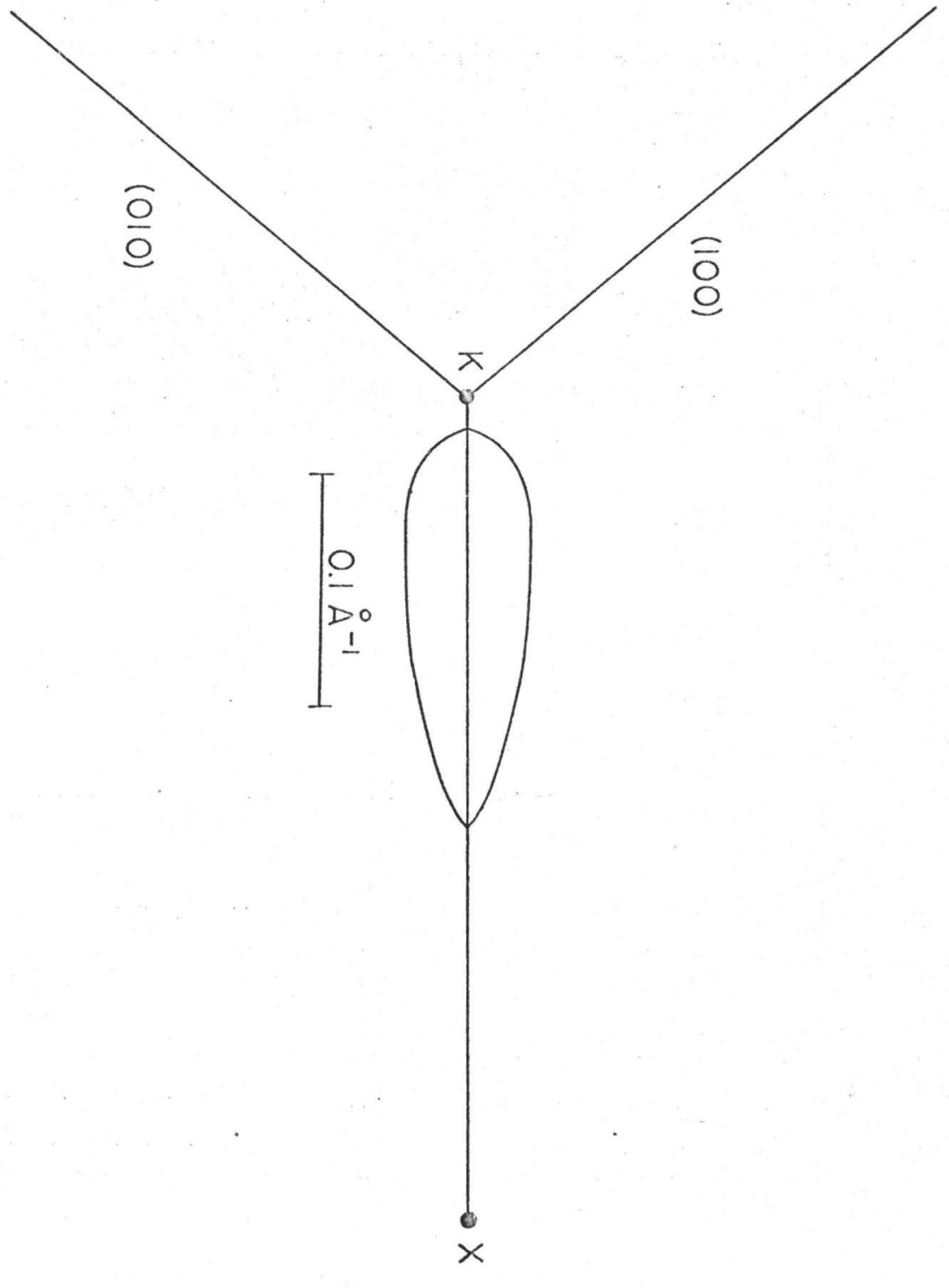

Figure (V.15) The minimum $B$ arm cross section for the local pseudopotential C $(0,0)$ 




Figure (V.16) The 'large section' of the lens for the local pseudopotential $\mathrm{C}(0,0)$ 


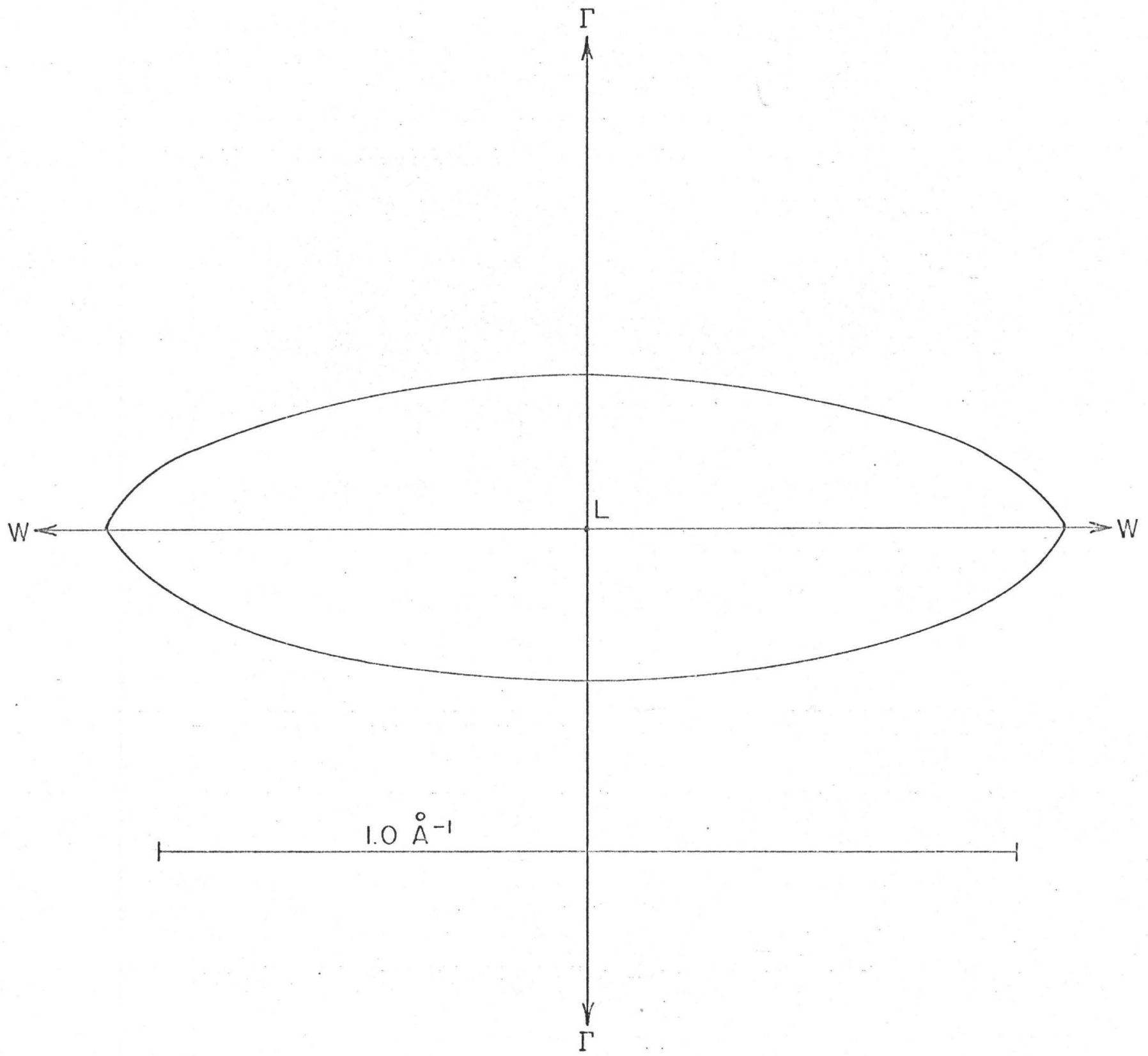

Figure (V.17) The 'up section' of the lens for the local. pseudopotential $\mathrm{C}(\mathrm{O}, \mathrm{O})$ 


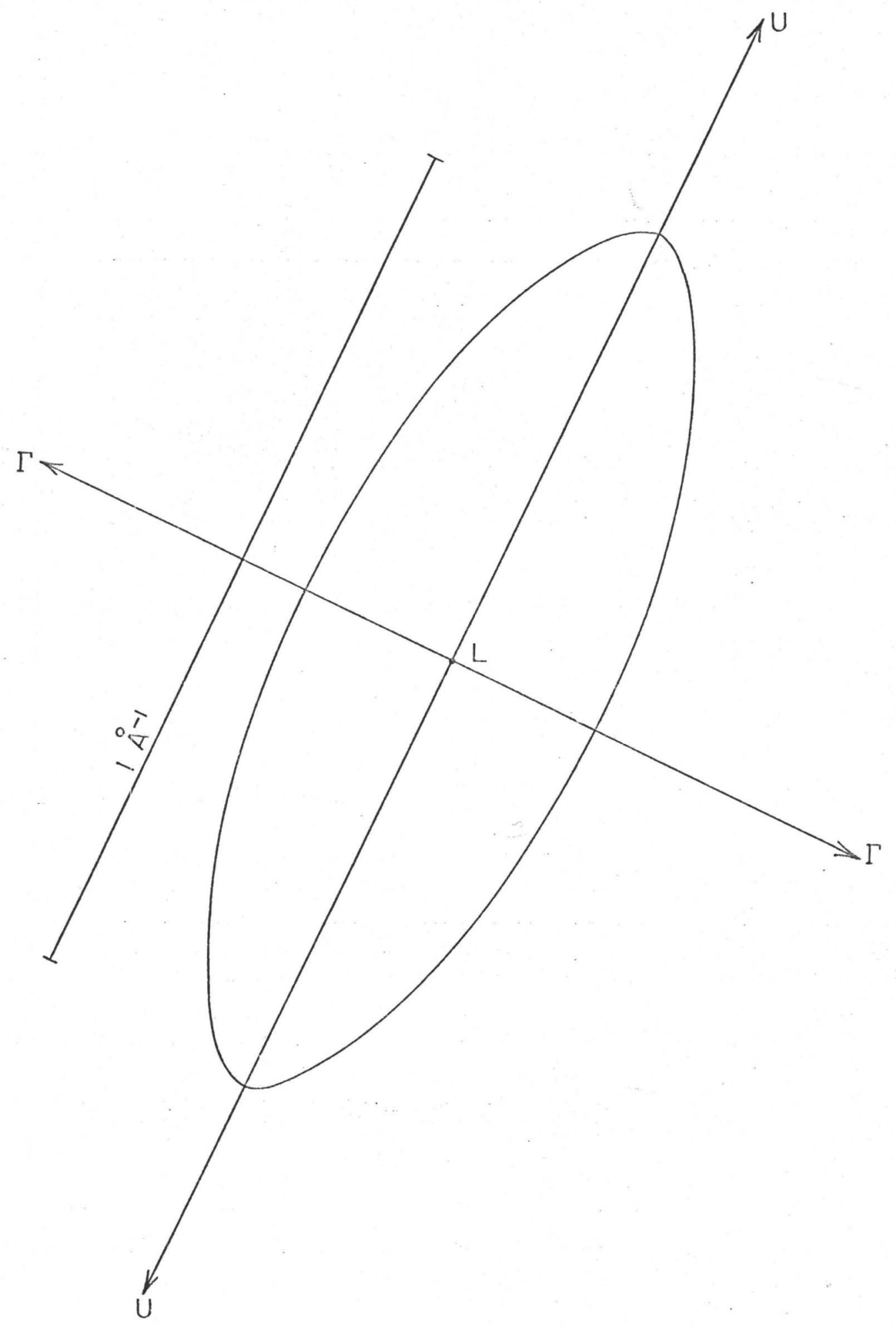

Figure (V.18) The 'side section' of the lens for the local pseudopotential $\mathrm{C}(0,0)$ 


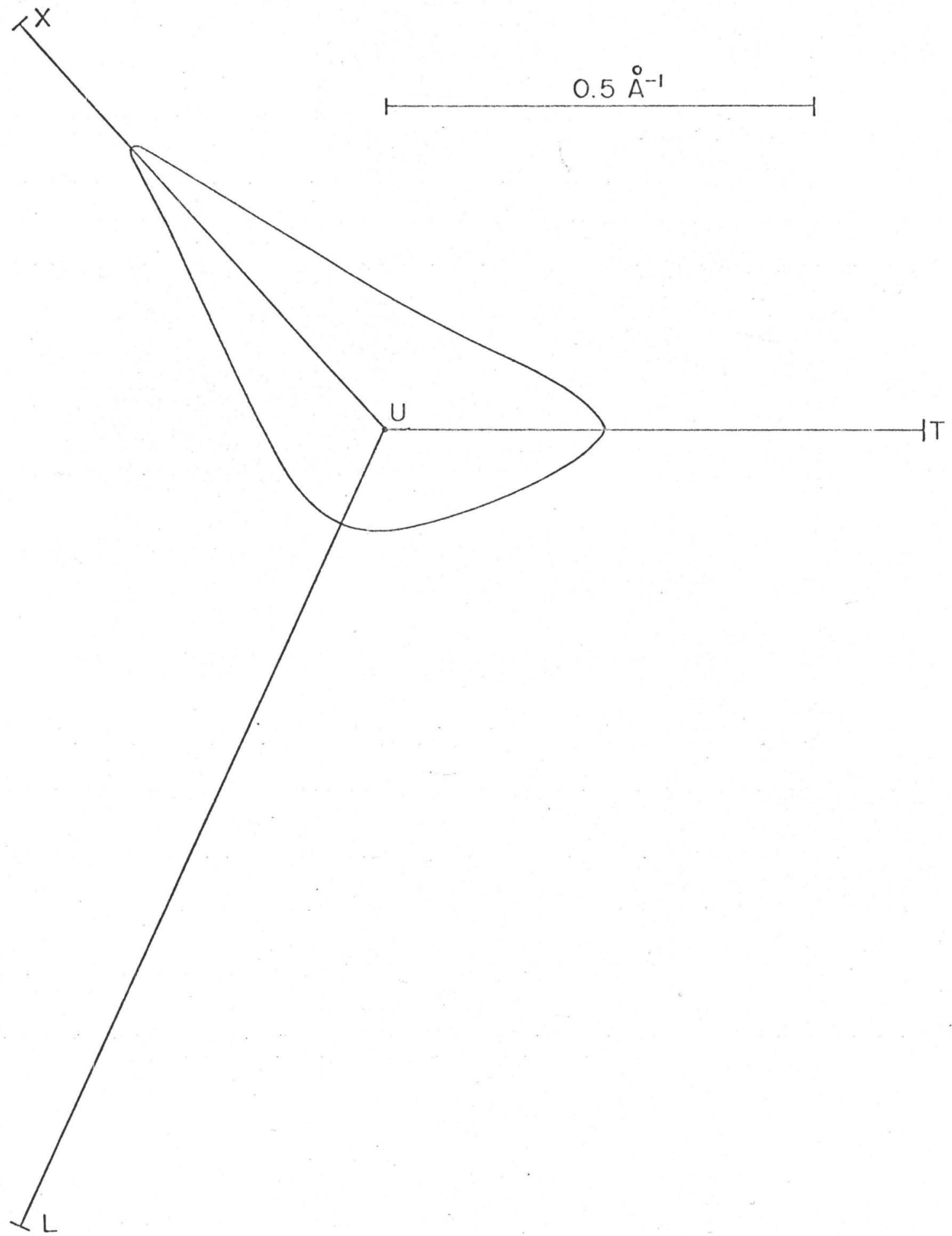
Figure (V.19) The 'minimum $\tau$ section' for the local pseuclo-
potential $C(0,0)$ 


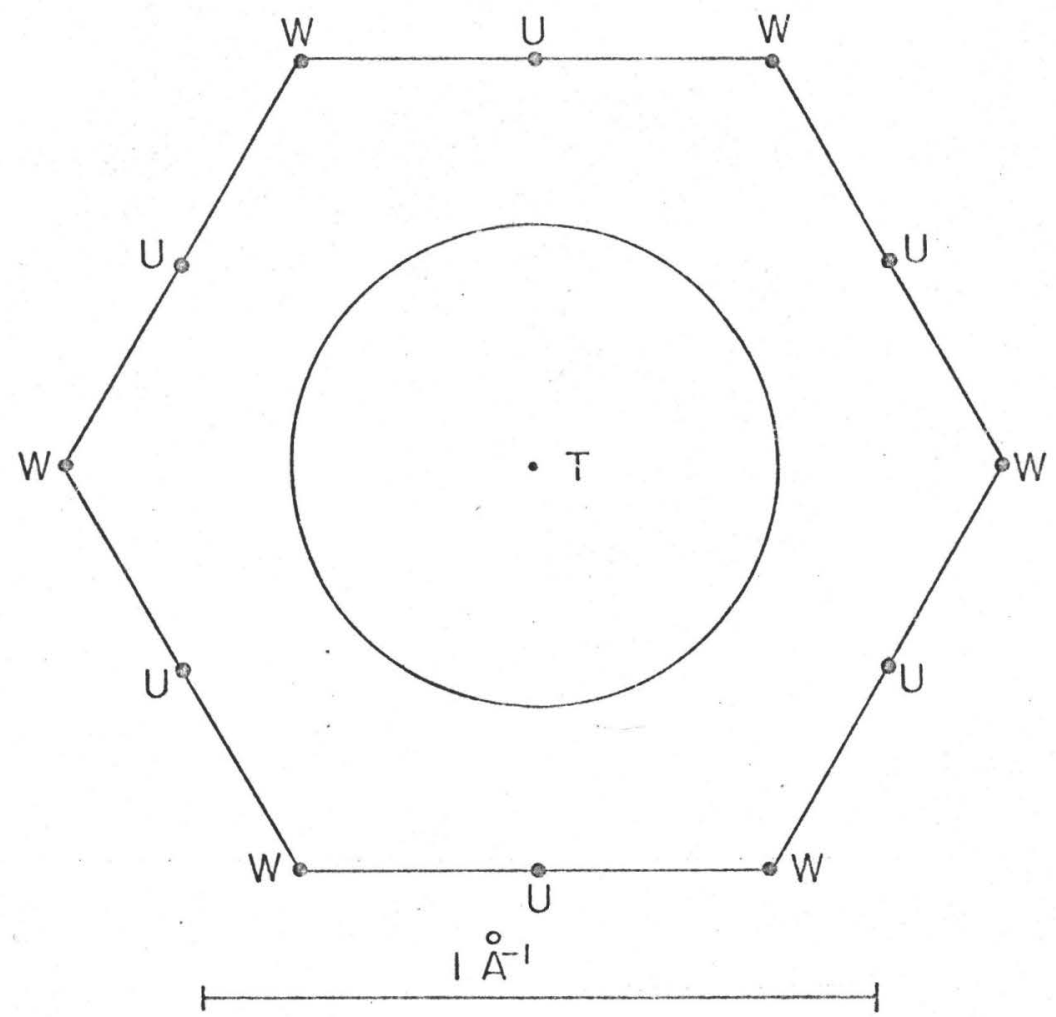

Figure (V.20) The 'T face opening' for the local pseudopotential $\mathrm{C}(\mathrm{O}, \mathrm{O})$ 




Figure (V.2l) The 'X face opening' for the local pseudopotential $c(0,0)$ 
probably not very accurate, but the table gives some idea of the orders of magnitude that were obtained.

\section{TABLE (V.8)}

Computed de Haas-van Alphen Frequencies and Cyclotron Masses for a Class B Pseudopotential

[In this table the values reported were obtained using a secular equation of approximate order $40 \times 40$, it was found that the convergence was not very good, and the figures listed have meaning only in an approximate order of magnitude sense.]

$$
\begin{aligned}
& \text { Parametrized Pseudopotential } \\
\mathrm{R} 5 \mathrm{~S}= & 6.8061, \mathrm{R} 5 \mathrm{P}=4.447, \mathrm{R} 5 \mathrm{D}=0.705 \\
\alpha= & 0.07, \beta=3.5, \gamma=1.5 \quad \delta=4.0 \\
& \text { Minimum B Arm Cross Sections }
\end{aligned}
$$

\begin{tabular}{|c|c|c|c|}
\hline \multicolumn{2}{|c|}{$E_{F}$ (Fermi energy) } & \multirow{2}{*}{$\begin{array}{l}\begin{array}{l}\text { Frequency } \\
\text { (Tesla) }\end{array} \\
46.628\end{array}$} & \multirow{2}{*}{$\begin{array}{l}\text { Cyclotron Mass } \\
\frac{\text { (reduced units) }}{-0.0556}\end{array}$} \\
\hline & 0.5720 & & \\
\hline & 0.5690 & 68.123 & -0.0678 \\
\hline \multicolumn{4}{|c|}{$\begin{array}{l}\text { [poor convergence encountered over part of } \\
\text { range, assumed Fermi energy by interpolation] } \\
\text { Sections for } E_{F}=0.5684 \text { Rydbergs }\end{array}$} \\
\hline orbit & & $\begin{array}{l}\text { Frequency } \\
\text { (Te'sla) }\end{array}$ & $\begin{array}{l}\text { Cyclotron Mass } \\
\text { (reduced units) }\end{array}$ \\
\hline Lens & (large section) & 9349 & 1.3397 \\
\hline Lens & (up section) & 2802 & 0.4143 \\
\hline Lens & (side section) & 2677 & 0.4005 \\
\hline
\end{tabular}


TABLE (V.8) continued

\begin{tabular}{lcc} 
Orbit & $\begin{array}{c}\text { Frequency } \\
\text { (Tesla) }\end{array}$ & $\begin{array}{c}\text { Cyclotron Mass } \\
\text { (reduced units) }\end{array}$ \\
${$\cline { 2 - 2 }$} }$ & 1512 & -0.3009 \\
T face & 2723 & 0.8478 \\
$X$ face & 2427 & 0.4970
\end{tabular}

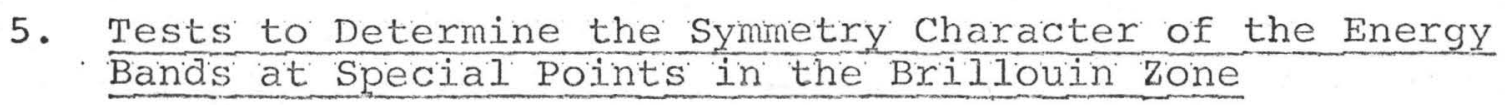

One method of testing the general symmetry character of the energy bands generated by a given pseudopotential is to calculate the energy bands and then to investigate the effect of increasing the $s, p$ and d-like parts of the nonlocal pseudopotential. Table (V.9) shows the results of such an analysis when applied to one of the class A pseudopotentials. The results show that the lowest level at $\Gamma$ is $s-l i k e$ while the second level is 's-d' like. At each of the points, T, $\mathrm{X}$, and $\mathrm{L}$ the lowest levels are 's-d' like and the second levels are p-like.

The symmetry analysis for the local pseudopotential $\mathrm{C}(0,0)$ is given in Table $(\mathrm{V}, \mathrm{IO})$. In this model the contact regions around $X$ and $T$ are $p-l i k e$ and the second zone lenses are also predominantly of p-like symmetry. A similar result holds for the Brandt-Rayne and the Heine-Animalu local pseudopotentials which also lie in this general area of parameter space. 
TABLE (У.9)

Symretry Analysis for a Class A Pseudopotential

Testing the symmetry character of the lowest energy bands at special points in the Brillouin zone.

\begin{tabular}{|c|c|c|c|c|c|}
\hline R5S & R5P & $\mathrm{R} 5 \mathrm{D}$ & $W_{L}$ & $\mathrm{~W}_{\mathrm{XT}}$ & $\mathrm{E}_{\mathrm{F}}(14$ points $)$ \\
\hline 0 & 3. & 1. & -0.004 & -0.012 & 0.6594 \\
\hline
\end{tabular}

The lowest energy levels at special symmetry points

\begin{tabular}{|c|c|c|c|c|c|c|c|}
\hline & R5S & R5P & $\mathrm{R} 5 \mathrm{D}$ & $\underline{\Gamma}$ & $\underline{T}$ & $\underline{L}$ & $\underline{x}$ \\
\hline $\begin{array}{l}\text { Zero per- } \\
\text { turbation }\end{array}$ & 0. & 3. & 1. & $\begin{array}{c}-0.00057 \\
1.4823\end{array}$ & $\begin{array}{l}0.6417 \\
0.7717\end{array}$ & $\begin{array}{l}0.4306 \\
0.5380\end{array}$ & $\begin{array}{l}0.6352 \\
0.7851\end{array}$ \\
\hline $\begin{array}{l}\text { Small s-like } \\
\text { perturbation }\end{array}$ & 0.1 & 3. & 1. & $\begin{array}{l}0.0133 \\
1.512\end{array}$ & $\begin{array}{l}0.6680 \\
0.7717\end{array}$ & $\begin{array}{l}0.4544 \\
0.5380\end{array}$ & $\begin{array}{l}0.6617 \\
0.7851\end{array}$ \\
\hline $\begin{array}{l}\text { Small p-like } \\
\text { perturbation }\end{array}$ & 0. & 3.1 & 1. & $\begin{array}{c}-0.00057 \\
1.4823\end{array}$ & $\begin{array}{l}0.6417 \\
0.7753\end{array}$ & $\begin{array}{l}0.4306 \\
0.5412\end{array}$ & $\begin{array}{l}0.6352 \\
0.7893\end{array}$ \\
\hline $\begin{array}{l}\text { Small d-like } \\
\text { perturbation }\end{array}$ & 0. & 3. & 1.1 & $\begin{array}{r}-0.00057 \\
3 . .4840\end{array}$ & $\begin{array}{l}0.6485 \\
0.7717\end{array}$ & $\begin{array}{l}0.4358 \\
0.5380\end{array}$ & $\begin{array}{l}0.6413 \\
0.7851\end{array}$ \\
\hline
\end{tabular}




\section{TABLE (V.10)}

Symmetry Analysis for a Class C Pseudopotential

Testing the symmetry character of the lowest energy bands at special points in the Brillouin zone.

\section{Pseudopotential (All energies in Rydbergs)}

\begin{tabular}{|c|c|c|c|c|c|}
\hline R5S & R5P & R5D & $\mathrm{w}_{\mathrm{L}}$ & $\mathrm{W}_{\mathrm{XT}}$ & $\mathrm{E}_{\mathrm{F}}(14$ points $)$ \\
\hline & 0. & 0. & -0.0825 & -0.0475 & 0.524 .7 \\
\hline
\end{tabular}

The lowest energy bands at special symmetry points

$\begin{array}{llllcccc} & \text { R5S } & \text { R5P } & \text { R5D } & \underline{I} & \underline{T} & \underline{I} & \underline{X} \\ \text { Zero } & 0 . & 0 . & 0 . & -0.0332 & 0.4517 & 0.2682 & 0.4750 \\ \text { perturbation } & & & & 1.3314 & 0.5910 & 0.4097 & 0.5250 \\ \text { small s-like } & 0.2 & 0 . & 0 . & -0.00239 & 0.4517 & 0.3089 & 0.4750 \\ \text { perturbation } & & & & 1.3314 & 0.6337 & 0.4097 & 0.5772 \\ \text { small p-like } & 0 . & 0.2 & 0 . & -0.0332 & 0.4791 & 0.2682 & 0.4999 \\ \text { perturbation } & & & & 1.3534 & 0.5910 & 0.4306 & 0.5250 \\ \text { small d-like } & 0 & 0 . & 0.2 & -0.0327 & 0.4517 & 0.2859 & 0.4750 \\ \text { perturbation } & & & & 1.3314 & 0.6109 & 0.4097 & 0.5428\end{array}$


The class B pesudopotentials differ from the previous example by having second zone lenses of essentially 's-d' symmetry. This is shown in Table (V.II) which gives the symmetry analysis for the pseudopotential whose band structure is illustrated in Figures (V.13) and (V.14). In this table the perturbations used are very strong.R5S, R5P, and R5D are given by $R 5 S=C S \times\left(E_{6 s}-E_{5 s}\right), R 5 P=C P \times\left(E_{6 s}-E_{5 p}\right)$, and $R 5 D=C D \times\left(E_{6 s}-E_{5 d}\right)$, (using the atomic energy levels of Herman and Skillman). In this paxticular example the local pseudopotential was given in the parametrized form of equation (5.1).

6. The Order of Magnitude of the Spin-orbit Interaction The spin-orbit term of the Hamiltonian in equation $(2.43)$ is

$$
W_{\text {so }}\left(\vec{k} S, \vec{k}^{\prime} S^{\prime}\right)=i\left[\lambda_{p}+\lambda_{d}\left(\underline{k} \cdot \underline{k}^{\prime}\right)\right]\left(\underline{k}{ }^{\prime} \times \underline{k} \cdot \sigma_{S S}{ }^{\prime}\right)
$$

where the parameters $\lambda_{p}$ and $\lambda_{d}$ arise from the $p$-like and-like core states respectively. Those conduction band states which contain a p-like component will be affected by $\lambda_{p}$, while $\lambda_{d}$ will affect states with a d-like component.

It is possible to estimate the size of the spin-orbit coupling parameters in mercury. The spin-orbit Hamiltonian for an atom can be wrjtten,

$$
H=\frac{p^{2}}{2 m}+V(\underline{r})+\frac{H}{2 m^{2} c^{2}} \underline{S} \cdot \nabla V \times \underline{p} .
$$

and

$$
V=\frac{r}{r} \frac{\partial V}{\partial r}
$$


TABLE (V.1I)

Symrnetry Analysis for a Class B Pseudopotential

Testing the symmetry character of the lowest energy bands at special points in the Brillouin zone.

\section{Pseudopotential (All energies in Rydbergs)}

$\begin{array}{lccccccccc}\underline{\alpha} & \underline{\beta} & \underline{Y} & \underline{\delta} & \underline{\mathrm{CS}} & \underline{\mathrm{CP}} & \underline{\mathrm{CD}} & \underline{\mathrm{R} 5 \mathrm{~S}} & \underline{\mathrm{R} 5 \mathrm{P}} & \underline{\mathrm{R} 5 \mathrm{D}} \\ 0.07 & 3.5 & 1.5 & 4.0 & 1 . & 1 . & 1 . & 6.8061 & 4.447 & 0.705\end{array}$

The lowest energy bands at special symmetry points

\begin{tabular}{|c|c|c|c|c|c|c|c|}
\hline & CS & $\mathrm{CP}$ & $C D$ & $\underline{I}$ & $\underline{T}$ & $\underline{L}$ & $\underline{x}$ \\
\hline $\begin{array}{l}\text { Zero } \\
\text { perturbation }\end{array}$ & 1. & 1. & 1. & $\begin{array}{l}0.2140 \\
1.547\end{array}$ & $\begin{array}{l}0.7553 \\
0.8715\end{array}$ & $\begin{array}{l}0.5609 \\
0.7213\end{array}$ & $\begin{array}{l}0.7737 \\
0.8446\end{array}$ \\
\hline $\begin{array}{l}\text { s-like } \\
\text { perturbation }\end{array}$ & 0. & I. & 1. & $\begin{array}{c}-0.3695 \\
1.051 \\
1.547\end{array}$ & $\begin{array}{l}0.2051 \\
0.7553\end{array}$ & $\begin{array}{r}-0.1315 \\
0.5609\end{array}$ & $\begin{array}{r}-0.0105 \\
0.7737\end{array}$ \\
\hline $\begin{array}{l}\text { p-like } \\
\text { perturbation }\end{array}$ & 1. & 0. & 1. & $\begin{array}{l}0.2140 \\
1.0339 \\
1.0339\end{array}$ & $\begin{array}{l}0.3410 \\
0.8305 \\
0.8305 \\
0.8715\end{array}$ & $\begin{array}{l}0.2070 \\
0.7213\end{array}$ & $\begin{array}{l}0.3369 \\
0.6020 \\
0.8446\end{array}$ \\
\hline $\begin{array}{l}\text { d-like } \\
\text { perturbation }\end{array}$ & 1. & 1. & 0. & $\begin{array}{l}0.2138 \\
1.2977 \\
1.2977\end{array}$ & $\begin{array}{l}0.7142 \\
0.7553\end{array}$ & $\begin{array}{l}0.5609 \\
0.6237\end{array}$ & $\begin{array}{l}0.6763 \\
0.7737\end{array}$ \\
\hline
\end{tabular}


This means that the spin-orbit term is given by

$$
W_{S O}=\frac{\hbar^{2}}{2 m^{2} c^{2}}\left(\frac{1}{r} \frac{\partial V}{\partial r}\right) \underline{S} \cdot \underline{l} \text {. }
$$

If a coulomb potential is assumed, so that

$$
V(r)=\frac{z e^{2}}{r} \text {, and } \frac{1}{r} \frac{\partial V}{\partial r}=\frac{z e^{2}}{r^{3}}
$$

it follows that spin-orbit effects are most important in heavy atoms, both on account of large $z$ and small $r$.

For zinc and cadmium, Stark and Falicov (1967) gave the following values for $\lambda_{p}$ and $\lambda_{d}$, (the units are in Rydbergs). Zinc $\left(z=30\right.$, configuration $\left.3 \mathrm{~d}^{10} 4 \mathrm{~s}^{2}\right), \lambda_{\mathrm{p}}=0.0050, \lambda_{\mathrm{d}}=0$. Cadmium $\left(z=48\right.$, configuration $\left.4 \mathrm{~d}^{10} 5 \mathrm{~s}^{2}\right), \lambda_{\mathrm{p}}=0.0105, \lambda_{\mathrm{d}}=0$. In the case of white tin the values are Craven (1969). $\left(z=50\right.$, configuration $\left.5 \mathrm{~s}^{2} 5 \mathrm{p}^{2}\right), \lambda_{\mathrm{p}}=0.013, \lambda_{\mathrm{d}}=0$.

In all of these examples it was sufficient to set $\lambda_{d}=0$. This is because the conduction bands are mostly 's-p' like. In the case of mercury also, Dishman and Rayne (1968) found that the inclusion of $\lambda_{d}$ produced a negligible effect and restricted their spin-orbit parameter to a $p$ like term.

There is no experimental information that can be used to give an estimate for $\lambda_{p}$ and $\lambda_{d}$ in mercury, however the atomic number of mercury is $\left(z=80\right.$, configuration $\left.5 \mathrm{~d}^{10} 6 \mathrm{~s}^{2}\right)$, and the trend of the previous figures for the elements zinc, cadmium, and white tin, suggest that $\lambda_{p} \simeq 0.02$ and $\lambda_{d}=0$ 
Rydbergs, might be a suitable approximation for these parameters.

Table (V.12) shows the effect of the spin-orbit coupling on the energy bands at special symmetry points in the Brillouin zone. In this table $\lambda_{p}$ is gradually increased in strength and $\lambda_{d}$ is zero. The local pseudopotential that is being used in Table $(\mathrm{V} .12)$ is the example $\mathrm{C}(0,0)$.

The secular equation is complex when spinmorbit coupling is included. There were no convenient subroutines available to diagonalize a complex Hamiltonian matrix of the form $(A+i B)$. Instead an unfolding technique was used and the real symmetric matrix $\left(\begin{array}{rr}A & B \\ -B & A\end{array}\right)$ was generated. This enlarged matrix has exactly the same eigenvalues as the original complex matrix, except that each eigenvalue repeats itself and occurs twice. The inclusion of spin increased the size of the secular equation from $(15 \times 15)$ to $(30 \times 30)$, which was then doubled by the unfolding technique.

\section{TABLE (V.12)}

The effect of spin-orbit coupling on a Class $C$ pseudopotential

The effect of the spin-orbit interaction on the energy bands at symmetry points is shown for different values of the spinorbit coupling parameter $\lambda_{p}$. 


\section{Pseudopotential}

$\mathrm{R} 5 \mathrm{~S}=0 ., \mathrm{R} 5 \mathrm{P}=0 ., \mathrm{R} 5 \mathrm{D}=0 ., \mathrm{W}_{\mathrm{L}}=-0.0825, \mathrm{~W}_{\mathrm{XT}}=0.0475$, $\lambda_{p}=0 ., \lambda_{d}=0 . \quad$ (all energies are in Rydbergs) Energy levels at symmetry points

I $\quad \underline{T} \quad \underline{W} \quad \frac{\text { L }}{\text { T }} \quad \underline{K} \quad \underline{\mathrm{X}} \quad \underline{\mathrm{U}}$ $\begin{array}{lllllll}-0.03304 & 0.4548 & 0.5604 & 0.2733 & 0.4836 & 0.4765 & 0.5288\end{array}$
$\begin{array}{lllllll}1.3339 & 0.5934 & 0.6008 & 0.4147 & 0.5152 & 0.5269 & 0.6407\end{array}$
$\begin{array}{lllllll}1.4138 & 1.1225 & 0.7506 & 1.4165 & 0.7029 & 0.9110 & 0.7178\end{array}$
$\begin{array}{lllllll}1.4138 & 1.2071 & 0.8268 & 1.4194 & 1.2680 & 0.9505 & 1.0209\end{array}$
$\begin{array}{lllllll}1.5278 & 1.2071 & 1.4371 & 1.4957 & 1.3452 & 1.3066 & 1.0879\end{array}$
$\begin{array}{lllllll}1.5278 & 1.3778 & 1.4592 & 1.5307 & 1.3963 & 1.3080 & 1.7544\end{array}$
$\begin{array}{llll}1.5733 & 1.3778 & 1.8691 & 1.7780\end{array}$
$1.5064 \quad 1.8948$

Including spin-orbit coupling

$\lambda_{p}=0.01$ Rydbergs, $\lambda_{d}=0$. Rydbergs

I $\quad \underline{T} \quad \underline{W} \quad \underline{L} \quad \underline{K} \quad \underline{X} \quad \underline{U}$

$\begin{array}{lllllll}-0.03304 & 0.4525 & 0.5558 & 0.2733 & 0.4828 & 0.4734 & 0.5250\end{array}$

$\begin{array}{lllllll}1.3171 & 0.5934 & 0.6020 & 0.4133 & 0.5131 & 0.5269 & 0.6404\end{array}$

$\begin{array}{lllllll}1.4138 & 1.1225 & 0.7500 & 1.4031 & 0.7024 & 0.9069 & 0.7176\end{array}$

$\begin{array}{lllllll}1.4138 & 1.1715 & 0.8272 & 1.4165 & 1.2644 & 0.9505 & 1.0191\end{array}$

$\begin{array}{lllllll}1.4870 & 1.2388 & 1.4303 & 1.4957 & 1.3384 & 1.3026 & 1.0879\end{array}$

$\begin{array}{lllllll}1.5665 & 1.3778 & 1.4628 & 1.5397 & 1.4009 & 1.3080 & 1.7403\end{array}$

$\begin{array}{llll}1.5733 & 1.3778 & 1.8679 & 1.7858\end{array}$

$1.5604 \quad 1.8948$ 


\begin{tabular}{|c|c|c|c|c|c|c|}
\hline I & $\underline{T}$ & $\underline{W}$ & I & $\underline{\mathrm{K}}$ & $\underline{x}$ & $\underline{U}$ \\
\hline 0.03304 & 0.4448 & 0.5415 & 0.2733 & 0.4789 & 0.4623 & 0.5115 \\
\hline 1.2533 & 0.5934 & 0.6050 & 0.4087 & 0.5069 & 0.5269 & 0.6396 \\
\hline 1.4138 & 1.1225 & 0.7489 & 1.3617 & 0.7009 & 0.8929 & 0.7174 \\
\hline 1.4138 & 1.3 .302 & 0.8282 & 1.4165 & 1.2499 & 0.9505 & 1.0136 \\
\hline 1.4617 & 1.2689 & 1.4136 & 1.4957 & 1.3246 & 1.2901 & 1.088 \\
\hline 1.5733 & 1.3778 & 1.4691 & 1.5595 & 1.4119 & 1.3080 & 1.7079 \\
\hline \multirow[t]{3}{*}{1.6013} & \multicolumn{2}{|l|}{1.3778} & \multicolumn{3}{|l|}{1.8652} & 1.7982 \\
\hline & \multicolumn{2}{|l|}{1.5066} & \multicolumn{3}{|l|}{1.8948} & \\
\hline & \multicolumn{6}{|c|}{$\lambda_{p}=0.03$ Rydbergs, $\lambda_{d}=0$ Rydbergs } \\
\hline$\underline{\Gamma}$ & $\underline{\underline{T}}$ & $\underline{W}$ & L & $\underline{k}$ & $\underline{x}$ & $\underline{U}$ \\
\hline-0.03304 & 0.4304 & 0.5170 & 0.2733 & 0.4662 & 0.4385 & 0.4849 \\
\hline 1.1465 & 0.5934 & 0.6087 & 0.4003 & 0.4999 & 0.5269 & 0.6386 \\
\hline 1.4138 & 1.0844 & 0.7476 & 1.3063 & 0.6990 & 0.8664 & 0.7172 \\
\hline 1.4138 & 1.1225 & 0.8297 & 1.4165 & 1.2188 & 0.9505 & 1.0042 \\
\hline 1.4546 & 1.2973 & 1.3895 & 1.4957 & 1.3139 & 1.2693 & 1.0881 \\
\hline 1.5732 & 1.3778 & 1.4752 & 1.5811 & 1.4246 & 1.3080 & 1.6616 \\
\hline 1.6323 & 1.3778 & & 1.861 .9 & & & 1.8098 \\
\hline & 1.5068 & & 1.8948 & & & \\
\hline
\end{tabular}

An investigation was also made into the effects of spin-orbit coupling on the band structure generated by the class A pseudopotentials. It was found that the inclusion of spin-orbit coupling did not rectify the main defect of this 
class of pseudopotentials which was the absence of $\beta$ arms. The energy levels shown in Table (V.12) show the effect of wave-function symmetry. All those states with a significant $p$-like component are perturbed when $\lambda_{p}$ is increased from zero. Double degeneracies are split apart by this component of the spin orbit coupling whenever the degenerate levels are p-like. All other energy levels remain unaffected. Near the Fermi surface most of these levels are 's-d'-jike in character with a dominant s-component. These levels will be affected by $\lambda_{d}$ if this parameter is increased from zero, however the s-like part of these states will not be affected by $\lambda_{d}$ and since this is the dominant component near the Fermi surface, $\lambda_{d}$ will probably not be a very important parameter.

7. Discussion

The results which have been presented show that the class A pseudopotentials are almost certainly spurious. They showed a persistent failure to generate $\beta$ arms and formed a continuum in parameter space with local pseudopotentials for which $W_{L}<0$ and $W_{X T}<0$. This failure of class $A$ lends support to class C because this set of pseudopotentials always generated a Fermi surface of the correct topology and formed a continum with those local pseudopotentials for which $W_{L}<0$ and $W_{X T}>0$, which is the region of the theoretical local pseudopotential given by Heine and Animalu. 
The class B pseudopotentials generate band structures and Fermi surfaces very similar to those given by class C. However in class B the second zone lenses have 's-a' symmetry whereas in class $\mathrm{C}$ they are p-like. This important difference between these two classes of pseudopotentials means that one of them must be wrong.

In Table ( $\mathrm{V} .5)$ there is a tendency for $\Delta \mathrm{E}$ to minimize in the region where the parameters are close to the approximate OPW values of $R 5 S=\left(E_{6 S}-E_{5 S}\right), R 5 P=\left(E_{6 s}-E_{5 p}\right)$, and $R 5 D=$ $\left(E_{6 s}-E_{5 d}\right)$. This suggests that class $B$ is an approximation for the OPW pseudopotential. The Fermi energy for this class was typically 0.57 Rydbergs, but it is possible that the bottom of the conduction band has been lifted by the 5 s operator by an amount that is too large relative to the top of the band. In Table (II.2) the repulsive potential of the $5 \mathrm{~s}$ state was listed as being equal to 0.9217 Rydbergs at $\underline{k}=0$. This repulsive potential applied for $\left(\mathrm{E}_{6 \mathrm{~S}}-\mathrm{E}_{5 \mathrm{~S}}\right)=6.806$ Rydbergs. The bottom of the conduction bands is approximately $10 \%$ nearer to the $5 \mathrm{~s}$ levels than is the Fermi level. This means that the Fermi energy just quoted of 0.57 Ryabergs could be enhanced up to 0.65 Rydbergs which is not far from the RAPW value of Keeton and Loucks which was at 0.68 Rydbergs.

In class $B$ the absolute energies were too high, but this was only because the local pseudopotential element W(o) was not negative enough. The Fermi surface is mostly deter- 
mined by $W_{L}, W_{X}$ and $W_{T}, W(0)$ only appears as a constant term in the diagonal elements of the secular matrix and its only function is to fix the origin for the energy bands.

The analysis given in Chapter II (section 9) was only approximate but the curve $\mathrm{W}_{\mathbf{S}}{ }^{*}(q)$ in Figure (II.3) suggests that appropriate values for $\mathrm{W}_{L}$ and $\mathrm{W}_{X T}$ in class $\mathrm{B}$ would be given by $\mathrm{W}_{\mathrm{L}}=-0.500$ Rydbergs, and $\mathrm{W}_{\mathrm{XT}}=-0.250$ Rydbergs. There is a region of mediocre fits in this area of parameter space but the Fermi energy is very low. When $W_{L}=-0.60$ Rydbergs, and $\mathrm{W}_{\mathrm{XT}}=-0.30$ Rydbergs there was an 8 point figure of merit of 39.5 with a Fermi energy of 0.4420 . Ryabergs. Using the earlier argument for the enhancement of the Fermi energy, the energy of 0.4420 Rydbergs could be increased up to 0.5300 Rydbergs which is near the free electron Fermi energy.

It is possible that this other region of parameter space in which the non-local terms are the same as in class $B$, but which has more negative local pseudopotential elements is the correct approximation for the OPW pseudopotential but this is not certain.

If this region is the correct approximation for the OPW pseudopotential it means that if a complete non-relativistic OPW calculation were carried out for mercury, that the conduction energy bands would have a Fermi energy near the free. electron level and the energy bands would resemble those generated by the local pseudopotentials of the Brandt-Rayne and 
Heine-Animalu type. If this is the case it means that these local pseudopotentials are nearly correct at their level of approximation but that they cannot account for the high Fermi energy generated by the RAPW calculation. Phenomenologically this high energy could be due to a combination of two factors: (i) hybridization of the $5 d$ band into the conduction band, and (ii) relativistic effects.

The pseudopotentials of class $C$ have the strongest resemblance to those used by Stark and Falicov in zinc and cadmium. Class $C$ pseudopotentials gave better agreement with the experimental cross sectional areas than did class $B$, although the calculations for this latter class were troublesome because of convergence difficulties. The inclusion of the non-local operators in class C produced some of the increase in Fermi energy that was looked for, but not so much as was obtained by Keeton and Loucks. The class C pseudopotentials appear to be the best for a non-local pseudopotential representation of the Fermi surface of mercury.

One problem that was encountered in this investigation was the great stability of the Fermi surface to changes in the pseudopotential parameters. Once a region of parameter space was located which generated approximately the correct Fermi surface, it was found that the parameters could be varied with quite a degree of latitude while the topology of the surface remained constant. This means that questions of methodology are very important for pseudopotential calcu- 
lations in mercury, and that the experimental data that is used in the search in parameter space should be as accurate as possible. It seems possible that the low resolving power of the method used in this investigation was because the experimental points used as a control were not accurate enough.

8. Comparison with Previous Calculations of the Fermi Surface

Table (V.13) compares the local pseudopotential parameters for several models.

Table (V.13)

Comparison of local pseudopotential coefficients different models.

$\begin{array}{llll}\text { Model } & \frac{\mathrm{W}_{100}}{-0.066} & \frac{\mathrm{w}_{110}}{0.047} & \frac{\mathrm{W}_{111}}{0.047} \\ \text { Brandt-Rayne } & -0.028 & 0.053 & 0.053 \\ \text { Heine-Animalu } & -0.0546 & 0.0616 & 0.0616 \\ \begin{array}{l}\text { Bogle, Coon, and } \\ \text { Grenier }\end{array} & -0.0825 & 0.0475 & 0.0475 \\ \mathrm{C}(0,0) & -0.045\end{array}$

Brandt and Rayne (1966) limited their pseudopotential to the simple local form and used only two parameters $\mathrm{W}_{\mathrm{L}}$ and $\mathrm{W}_{\mathrm{XT}}$. In their calculations the secular equations never exceeded $(6 \times 6)$, and the parameters were obtained by fitting to the $\alpha, \beta$, and $\tau$ orbits.

The pseudopotential of Bogle, Coon, and Grenier (1969) was derived from a fit to the second zone lenses determined from magneto-acoustic calipers. This calculation was limited 
to four plane waves and there is some evidence that the convergence was not good. For example two different values for the $\mathrm{T}$ face opening were reported. A minimum value of $\mathrm{k}_{\mathrm{IN}}^{\mathrm{T}-\mathrm{U}}=$ $0.270 \AA^{-1}$, and a maximum value of $\mathrm{k}_{\mathrm{IN}}^{\mathrm{T}-\mathrm{W}}=0.340 \AA-1$. This large deviation from a circle probably resulted from the use of a small secular equation.

Table (V.I4) compares some of the extremal frequencies predicted by various theoretical models.

TABLE (V.14)

Comparisons of extremal frequencies for model Fermi surfaces with experimental aHvA areas

Model

$\begin{array}{lccc} & \underline{\alpha} & \underline{\beta} & \underline{\tau} \\ \text { Experimental } & 3240 & 74 & 1580 \\ \text { Brandt-Rayne } & 3600 & 74 & 1120 \\ \text { RAPW } & 3706 & 74 & 1382 \\ 8 \mathrm{PW} \text { (Dishman-Rayne) } & 4429 & 74 & 1580 \\ \mathrm{C}(\mathrm{O}, \mathrm{O}) & 3052 & 74 & 1126\end{array}$

\section{Extremal frequency (tesla)}

.


$\tau$ orbits to be too small except for Dishman and Rayne (1968) who used the orbit for fitting. Dishman and Rayne included spin-orbit coupling using a formalism due to Animalu (1966). Their final fitting parameters were obtained by a fit to the first zone hole surface, and their second zone lenses were much too large. In their RAPW calculation Keeton and Loucks set the Fermi energy equal to 0.680 Rydbergs by making the $\beta$ arms with the correct cross section.

In Table (V.13) the different values of $\mathrm{W}_{110}$ and $\mathrm{W}_{111}$ do not differ very much from each other. However there are large variations in $\mathrm{W}_{100^{\circ}}$. These variations are related to the different sizes of the second zone lens. In the free electron model the second zone lens is too large, and when $\left|W_{L}\right|$ is small the lens remains large. As $W_{L}$ increases in absolute value the lenses become smaller. It seems that the result $\mathrm{W}_{\mathrm{I}}=-0.066$ which was obtained by Brandt and Rayne was not quite negative enough. On the other hand the value of $\mathrm{W}_{\mathrm{L}}=-0.0825$ Rydbergs given by pseudopotential $\mathrm{C}(0,0)$ may be slightly too negative. It appears that at the level of the local approximation

$$
-0.066>W_{L}>-0.0825 \text { Rydbexgs. }
$$

This correlates with the observation of Dishman and Rayne that they found exact agreement with the minimum area of the lens when $\mathrm{W}_{\mathrm{L}}=-0.074$ Rydbergs. 


\section{CHAPTER VI \\ CONCLUSION}

The pseudopotential approximation gives a theoretical model of the Fermi surface of mercury that agrees fairly well with experiment. A surface is produced which has the correct topology although it does not appear possible to produce exact quantitative agreement with all the experimental dimensions of the Fermi surface. The pseudopotential energy bands resemble the free electron bands to some extent but most of the accidental degeneracies are removed. There are some doubly degenerate energy levels on the trigonal axis which are a consequence of the crystal symmetry but the levels are split when the spin-orbit interaction is taken into account. The only degeneracy that remains is the spin degeneracy which is a consequence of time reversal symmetry and of the inversion symmetry of the rhombohedral lattice.

There is a tendency for the two lowest energy bands to run parallel over the central regions of the zone faces. This is explained by simple perturbation theory. Group theory can be used to derive approximate expressions for the energy levels at symmetry points.

The inclusion of non-local operators into the pseudo- 
potential affects different parts of the energy bands and Fermi surface in different ways depending upon the symmetry of the bands. A non-local operator with a given angular momentum character perturbs the bands in those regions where the wavefunctions possess a significant component having the same angular momentum. When this is not the case, the non-local operator has little or no effect. This property can be used to test the general symmetry characteristics of the energy bands.

The spin-orbit coupling operators are also characterized by their angular momentum, and these operators perturb the energy bands and split residual degeneracies whenever the wavefunctions possess a component with the same angular momentum.

The non-local operators have a strong influence on the value of the Fermi energy. When a simple local pseudo.. potential is used the Fermi level is close to the free electron value of 0.5240 Ryabergs relative to the bottom of the conduction band. The p-like and d-like non local operators cause the Fermi energy to increase. The s-like non-local operator acts in the opposite sense and causes a decrease by raising the bottom of the band. This occurs because the electron states of the Fermi surface contain significant higher angular momentum components, but the bottom of the conduction band is almost entirely s-like. 
There are three regions of parameter space where the pseudopotentials generate a Fermi surface with a good fit to the experimental magneto-acoustic calipers reported by Bogle, Coon, and Grenier (1969).

The class A nonmlocal pseudopotentials generate second zone lenses, and $\mathrm{x}$ and $\mathrm{T}$ face contact regions, but the lowest energy band between $\mathrm{x}$ and $\mathrm{K}$ is too low in energy to generate $\beta$ arms. The band is also extremely flat in this region. This implies heavy cyclotron masses for the $\beta$ orbits but experimentally these are known to be light.

The class $B$ and class $C$ non-local pseudopotentials generate a Fermi surface with the correct topology, but class B possesses second zone lenses of ' $s-d$ ' symmetry and class C has lenses of p-like symmetry. The class C pseudopotentials had the better fits to the experimental dHvA frequencies. This class also included the region of parameter space. given by the treoretical model of Heine and Animalu. The question of which is the best member of class $C$ depends upon the criterion adopted. The local pseudopotential $\mathrm{C}(0,0)$ has the best $f i t$ to the magneto-acoustic data, $C(1,0), C(1,1)$, and $C(1,2)$ have better agreement to the $\tau$ orbits. $\mathrm{C}(2,2)$ has a Fermi energy of 0.6830 Rydbergs which is almost the same as the RAPW value of 0.680 Rydbergs.

The ambiguities and uncertainties which exist are partially due to a lack of sufficient information, in particular 
there is a need for an experimental value of the Fermi energy in mercury. More accurate data specifying the Fermi surface would be useful, although achieving a fit to the Fermi surface is not sufficient. It is also necessary to have the correct band structure, which might be obtained from optical measurements. The experimental values of the energy gaps at the symmetry points would be useful for fixing the pseudopotential parameters.

If this information becomes available classes $B$ and $C$ should be re-investigated in the regions indicated in Chapter $V$, with the inclusion of spin-orbit coupling. 


\section{BIBLIOGRAPHY}

I. Abarenkov, and V. Heine (1965), Phil. Mag. 12, 529.

A. O. E. Animalu (1966), Phil. Mag. 13, 53.

A. O. E. Animalu and V. Heine (1965), Phil. Mag. 12, 1249.

B. J. Austin, V. Heine, and L. J. Sham (1962), Phys. Rev. 127 276 .

T. E. Bogle, J. B. Coon, and C. G. Grenier (1969), Phys. Rev. 177, No. 3, 1122 .

G. B. Brandt and J. A. Rayne (1966), Phys. Rev. 148, 644.

D. Brust (1964), Phys. Rev. 134, Al337.

M. I. Cohen and T. K. Bergstresser (1966), Phys. Rev. 141, 789.

M. L. Cohen, L. M. Falicov, and S. Golin (1964), I.B.M. J. Res. Rev. 8, 215.

J. E. Craven (1969), Phys. Rev. 182, 693.

W. R. Datars and A. E. Dixon (1967), Phys. Rev. 154, No. 3, 576.

J. M. Dishman and J. A. Rayne (1968), Phys. Rev. 166, No. 3, 728 .

A. E. Dixon and W. R. Datars (1968), Phys. Rev. 175, No. 3, 928.

L. M. Falicov and S. Golin (1965), Phys. Rev. 137, 3A, A871.

W. A. Harrison (1962), Phys, Rev, 126, 497.

F. Herman and S. Skillman, 'Atomic Structure Calculations (Prentice Hall, Inc:, Englewood Cliffs, New Jersey, 1965).

C. Herring (1940), Phys. Rev. 57, 1169. 
H. Jones, 'The Theory of Brillouin Zones and Electronic States in Crystals, (North Holland Publishing Company, Amsterdam, 1962)'.

S. C. Keeton, and T. L. Loucks (1966), Phys. Rev. 152, 548.

J. C. Kimball, R. W. Stark, and F. M. Mueller (1967), Phys. Rev. 162, No. 3, 600 .

P. J. Lin, and L. M. Falicov (1966), Phys. Rev. 142, 441.

T. L. Loucks (1966), Phys. Rev. 143, 506.

L. F. Mattheiss (1964), Phys. Rev. 134, A970.

J. S. Moss (1968), Ph.D. Thesis, McMaster University.

J. C. Phillips, and L. Kleinman (1959), Phys. Rev. 116, 287, 880.

R. Pick, and G. Sarma (1964), Phys. Rev. 135, Al363.

R. G. Poulsen (1970), Ph.D. Thesis, McMaster University

R. G. Poulsen, J. S. Moss, and W. R. Datars (1970) to be published.

M. G. Priestley, L. R. Windmiller, J. B. Ketterson, and Y. Ekstein (1967), Phys. Rev. 154, 671.

R. W. Stark, and L. M. Falicov (1967), Phys. Rev. Letters, Vol. 19, number 14, 795.

M. Tinkham, 'Group theory and quantum mechanics, (McGraw-Hill Book Company, 1964)'

G. Weisz (1966), Phys. Rev. 149, Number 2, 504.

E. G. Wilson and S. A. Rice (1966), Phys. Rev. 145, 55. 